\title{
Radical C-C Bond Cleavage/Addition Cascade of Benzyl Cycloketone Oxime Ethers Enabled by Photogenerated Cyclic Iminyl Radicals
}

\author{
Peng-Zi Wang, ${ }^{\dagger}$ Bin-Qing He, ${ }^{\dagger}$ Ying Cheng, ${ }^{\dagger}$ Jia-Rong Chen, ${ }^{*, \dagger}$ and Wen-Jing Xiao ${ }^{*},+$,
}

${ }^{\dagger}$ CCNU-uOttawa Joint Research Centre, Hubei International Scientific and Technological Cooperation Base of Pesticide and Green Synthesis, Key Laboratory of Pesticides \& Chemical Biology Ministry of Education, College of Chemistry, Central China Normal University, 152 Luoyu Road, Wuhan, Hubei 430079, China

\$ State Key Laboratory of Applied Organic Chemistry, Lanzhou University, Lanzhou 730000, China E-mail: chenjiarong@mail.ccnu.edu.cn; wxiao@mail.ccnu.edu.cn

\section{Table of Contents}

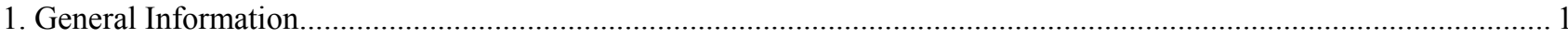

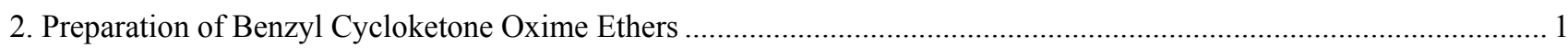

2.1 Preparation of Benzyl Cycloketone Oxime Ethers 1a-1g, 1k-1q ............................................................... 1

2.2 Preparation of Benzyl Cyclobutanone Oxime Ethers 1g-1j............................................................... 2

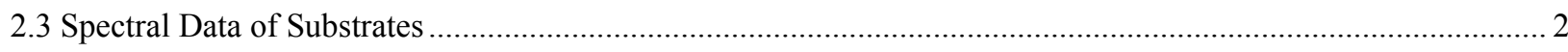

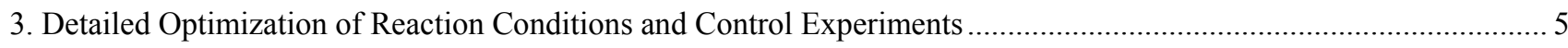

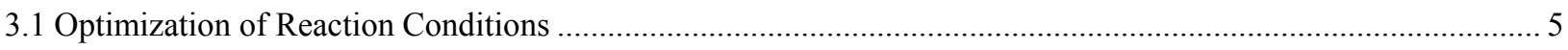

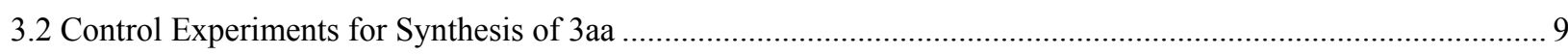

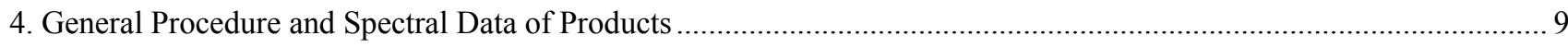

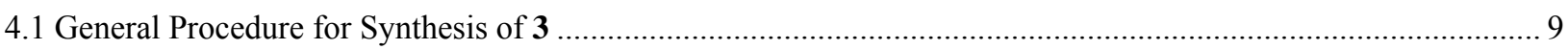

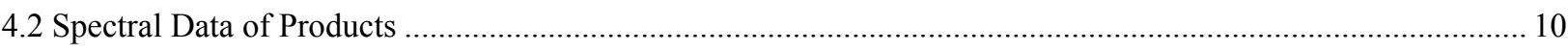

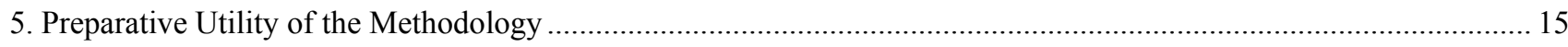

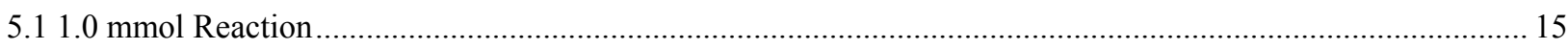

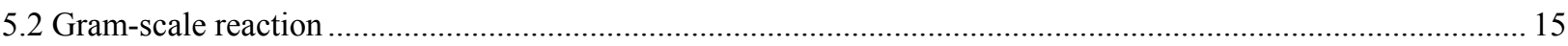

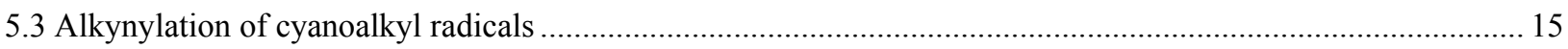

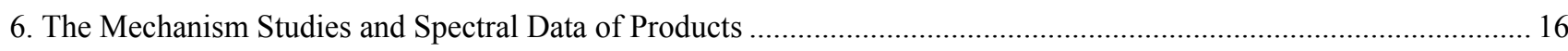

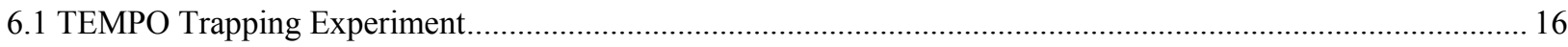

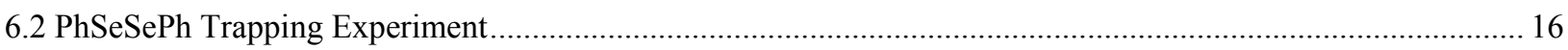

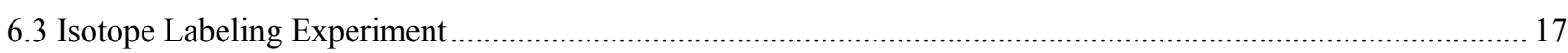

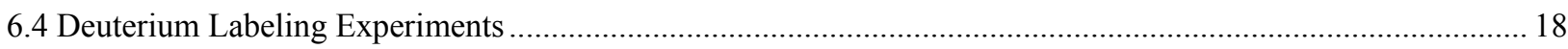

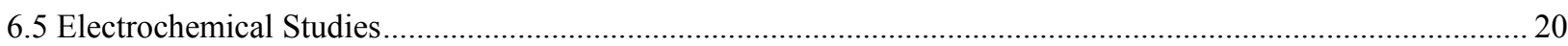

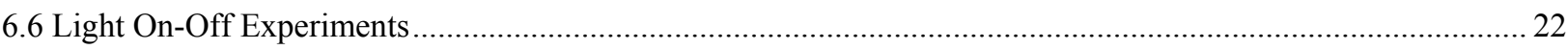

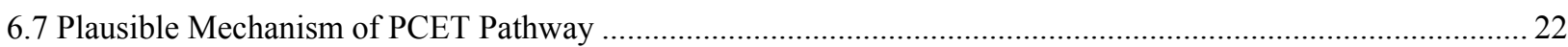

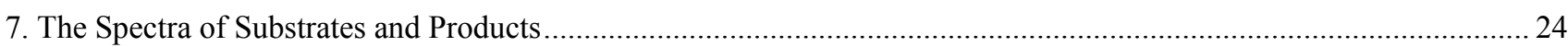

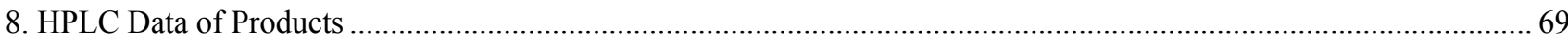




\section{General Information}

Unless otherwise noted, materials were purchased from commercial suppliers and used without further purification. All the solvents were treated according to general methods. Flash column chromatography was performed using 200-300 mesh silica gel. ${ }^{1} \mathrm{H}$ NMR spectra were recorded on 400 or $600 \mathrm{MHz}$ spectrophotometers. Chemical shifts $(\delta)$ are reported in ppm from the resonance of tetramethyl silane as the internal standard (TMS: $0.00 \mathrm{ppm}$ ). Data are reported as follows: chemical shift, multiplicity $(\mathrm{s}=$ singlet, $\mathrm{d}=$ doublet, $\mathrm{t}=$ triplet, $\mathrm{q}=$ quartet, $\mathrm{m}=$ multiplet), coupling constants $(\mathrm{Hz})$ and integration. ${ }^{13} \mathrm{C}$ NMR spectra were recorded on 100 or $150 \mathrm{MHz}$ with complete proton decoupling spectrophotometers. The high resolution mass spectra (HRMS) were measured on Bruker micrOTOF II by ESI. Unless otherwise noted, materials obtained from commercial suppliers were used without further purification.

\section{Preparation of Benzyl Cycloketone Oxime Ethers}

\subsection{Preparation of Benzyl Cycloketone Oxime Ethers 1a-1g, 1k-1q}

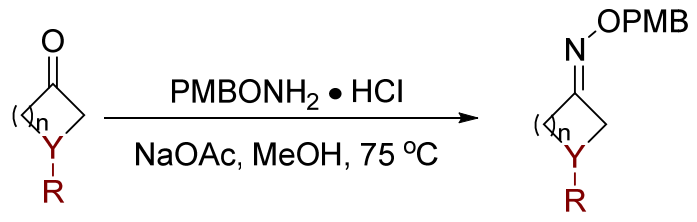

1a-1g, 1k-1q

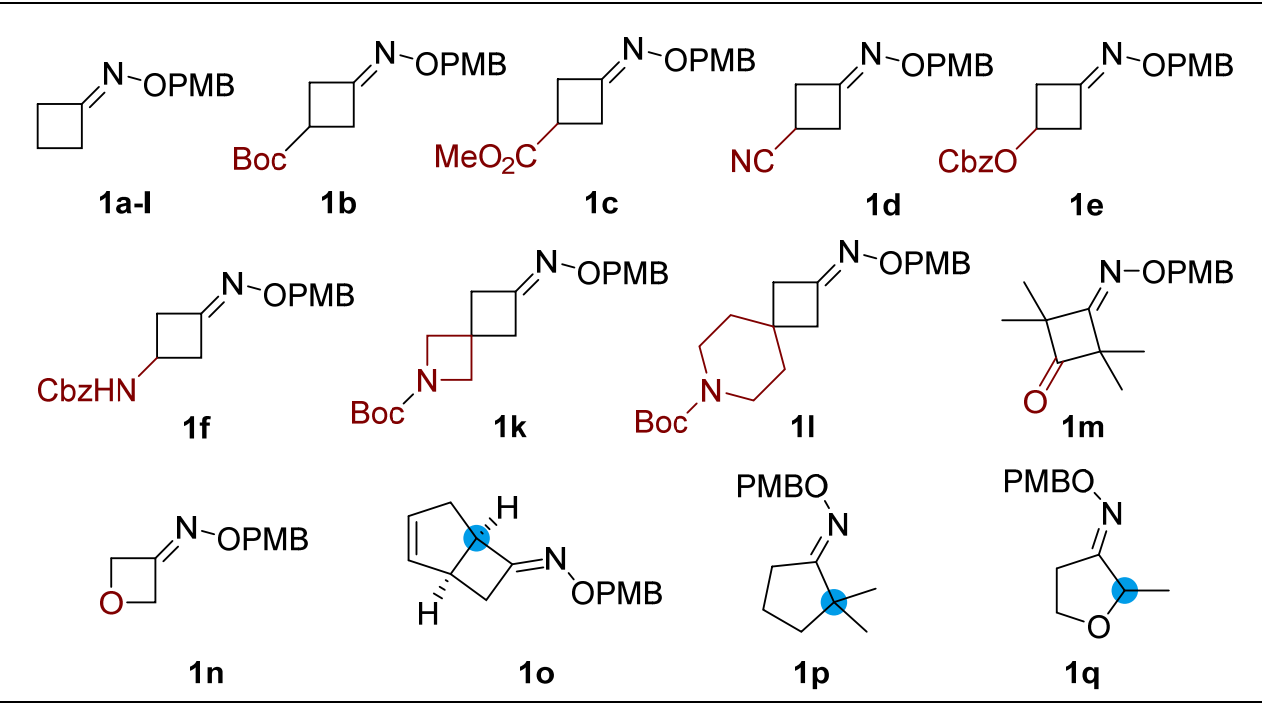

The hydroxylamine hydrochloride was prepared according to the literature.

To hydroxylamine hydrochloride (1.2 equiv) and sodium acetate (1.2 equiv) was added the solution of ketone in $\mathrm{MeOH}(0.3 \mathrm{M})$. The reaction mixture was warmed up to $75{ }^{\circ} \mathrm{C}$ and stirred overnight. The resulting mixture was cooled dawn to room temperature, diluted with brine and EtOAc and extracted with EtOAc. The organic layer was dried over $\mathrm{Na}_{2} \mathrm{SO}_{4}$ and concentrated in vacuo. Purification of the crude product by column chromatography on silica gel provided the oxime esters. 


\subsection{Preparation of Benzyl Cyclobutanone Oxime Ethers 1g-1j}

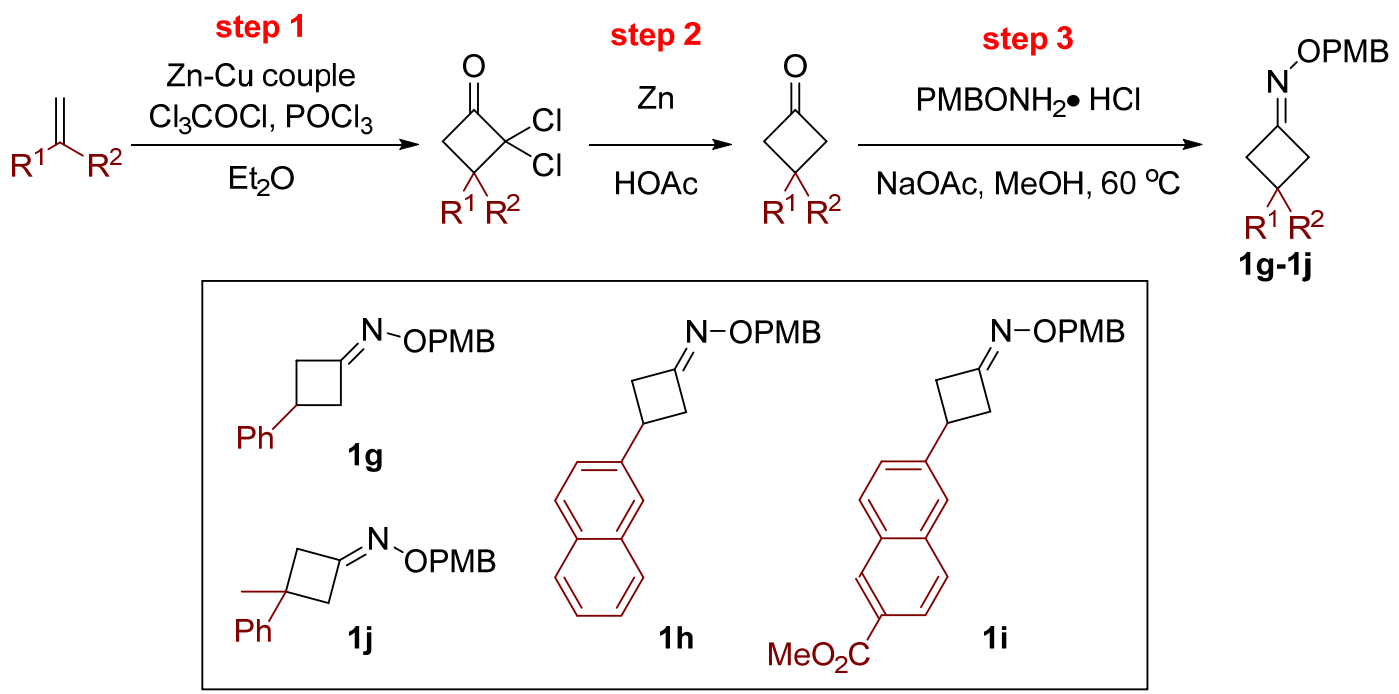

To a $250 \mathrm{~mL}$ three-necked flask under argon were added alkene derivative ${ }^{2}$ (1.0 equiv), zinc-copper couple (3.0 equiv), and anhydrous ether $(0.5 \mathrm{M})$. To this was added a solution of trichloroacetyl chloride (2.0 equiv) and phosphorus oxychloride (1.1 equiv) in ether $(0.5 \mathrm{M})$ over $1 \mathrm{~h}$ through an addition funnel. The suspension was stirred overnight at reflux. The resulting mixture was filtered through a pad of Celite and was washed with ether $(80 \mathrm{~mL})$. The organic solution was successively washed with water $(30 \mathrm{~mL})$, a saturated aqueous solution of $\mathrm{NaHCO}_{3}(30 \mathrm{~mL})$ and brine $(30$ $\mathrm{mL}$ ), and dried over $\mathrm{Na}_{2} \mathrm{SO}_{4}$. Then the solution was filtered, concentrated and used in the next step without further purification.

A mixture of 2,2-dichlorocyclobutanones (1.0 equiv) and zinc dust (4.0 equiv) in acetic acid (10 mL) was stirred at room temperature for $2 \mathrm{~h}$ and then heated at $80{ }^{\circ} \mathrm{C}$ for $5 \mathrm{~h}$. The resulting mixture was allowed to cool to room temperature, then, the solution was diluted with water $(30 \mathrm{~mL})$ and extracted with ether $(3 * 20 \mathrm{~mL})$. The organic phase was washed successively with a saturated solution of aqueous $\mathrm{NaHCO}_{3}(3 * 30 \mathrm{~mL})$, water $(30 \mathrm{~mL})$ and brine $(30 \mathrm{~mL})$, then dried over $\mathrm{Na}_{2} \mathrm{SO}_{4}$ and concentrated in vacuum. The crude material was then purified by flash chromatography with a mixture of petroleum ether and ethyl acetate to afford various cyclobutanones.

The following steps are same as the mentioned above.

\subsection{Spectral Data of Substrates}

\section{cyclobutanone O-(4-methoxybenzyl) oxime 1a-I}

N-OPMB 1a-I was prepared from the corresponding cyclobutanone $(5.0 \mathrm{mmol})$ as colorless oil $(690 \mathrm{mg}, 64 \%) .{ }^{1} \mathrm{H}$ NMR (400 MHz, CDCl $) \delta 7.30(\mathrm{~d}, J=8.28 \mathrm{~Hz}, 2 \mathrm{H}), 6.88(\mathrm{~d}, J=7.90 \mathrm{~Hz}, 2 \mathrm{H}), 4.96(\mathrm{~s}, 2 \mathrm{H}), 3.80(\mathrm{~s}, 3 \mathrm{H})$, $2.91(\mathrm{t}, J=8.72 \mathrm{~Hz}, 3 \mathrm{H}), 2.01-1.93(\mathrm{~m}, 2 \mathrm{H}) .{ }^{13} \mathrm{C} \mathrm{NMR}\left(100 \mathrm{MHz}, \mathrm{CDCl}_{3}\right) \delta 159.3,159.2,130.2,129.8$, 113.8, 77.4, 77.1, 76.8, 75.3, 55.3, 31.7, 31.3, 14.6. HRMS (EI): $\mathrm{m} / \mathrm{z}[\mathrm{M}+\mathrm{Na}]^{+}$calcd for $\mathrm{C}_{12} \mathrm{H}_{15} \mathrm{NNaO}_{2}$ : 228.0995, found: 228.0995 .

\section{tert-butyl 3-(((4-methoxybenzyl)oxy)imino)cyclobutane-1-carboxylate 1b}

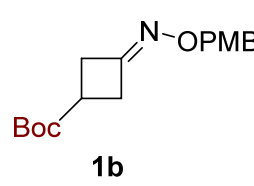

calcd for $\mathrm{C}_{17} \mathrm{H}_{13} \mathrm{NNaO}_{4}$ : 328.1519 , found: 328.1520 .

1b was prepared from the corresponding cyclobutanone $(5.0 \mathrm{mmol})$ as white soild $(1.20 \mathrm{~g}, 80 \%)$. ${ }^{1} \mathrm{H}$ NMR (400 MHz, $\left.\mathrm{CDCl}_{3}\right) \delta 7.29(\mathrm{~d}, J=8.63 \mathrm{~Hz}, 2 \mathrm{H}), 6.88(\mathrm{~d}, J=8.66 \mathrm{~Hz}, 2 \mathrm{H}), 4.97$ (s, 2H), 3.79 (s, $3 \mathrm{H}), 3.12-3.01(\mathrm{~m}, 5 \mathrm{H}), 1.45(\mathrm{~s}, 9 \mathrm{H}) .{ }^{13} \mathrm{C} \mathrm{NMR}\left(100 \mathrm{MHz}, \mathrm{CDCl}_{3}\right) \delta 173.2,159.4,154.3,130.0$, $129.9,113.8,81.0,77.5,77.1,76.8,75.5,55.3,35.5,35.1,32.3,28.0$. HRMS (EI): $\mathrm{m} / \mathrm{z}[\mathrm{M}+\mathrm{Na}]^{+}$ 
methyl 3-(((4-methoxybenzyl)oxy)imino)cyclobutane-1-carboxylate 1c

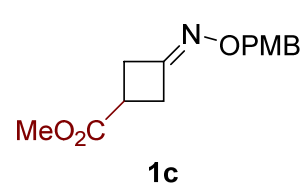

1c was prepared from the corresponding cyclobutanone $(5.0 \mathrm{mmol})$ as colorless oil $(1.02 \mathrm{~g}, 77 \%)$. ${ }^{1} \mathrm{H}$ NMR (400 MHz, $\left.\mathrm{CDCl}_{3}\right) \delta 7.28(\mathrm{~d}, J=8.41 \mathrm{~Hz}, 2 \mathrm{H}), 6.87(\mathrm{~m}, J=8.51 \mathrm{~Hz}, 2 \mathrm{H}), 4.96(\mathrm{~s}, 2 \mathrm{H})$, $3.78(\mathrm{~s}, 3 \mathrm{H}), 3.70(\mathrm{~s}, 3 \mathrm{H}), 3.24-3.08(\mathrm{~m}, 5 \mathrm{H}) .{ }^{13} \mathrm{C} \mathrm{NMR}\left(100 \mathrm{MHz}, \mathrm{CDCl}_{3}\right) \delta 174.4,159.4,153.7$, $129.9,113.8,77.5,77.2,76.9,75.6,55.2,52.2,35.6,35.2,31.2 . \mathrm{HRMS}(\mathrm{EI}): \mathrm{m} / \mathrm{z}[\mathrm{M}+\mathrm{Na}]^{+}$calcd for $\mathrm{C}_{14} \mathrm{H}_{17} \mathrm{NNaO}_{4}$ : 286.1050, found: 286.1054 .

\section{3-(((4-methoxybenzyl)oxy)imino)cyclobutane-1-carbonitrile 1d}

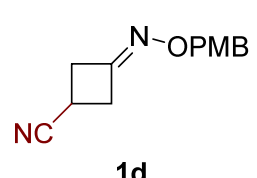

$1 d$

1d was prepared from the corresponding cyclobutanone $(5.0 \mathrm{mmol})$ as colorless oil $(986 \mathrm{mg}, 86 \%)$. ${ }^{1} \mathrm{H}$ NMR (400 MHz, $\mathrm{CDCl}_{3}$ ) $\delta 7.27$ (d, $\left.J=8.41 \mathrm{~Hz}, 2 \mathrm{H}\right), 6.88(\mathrm{~d}, J=8.45 \mathrm{~Hz}, 2 \mathrm{H}), 4.97$ (s, 2H), 3.79 $(\mathrm{s}, 3 \mathrm{H}), 3.37-3.09(\mathrm{~m}, 5 \mathrm{H}) .{ }^{13} \mathrm{C} \mathrm{NMR}\left(100 \mathrm{MHz}, \mathrm{CDCl}_{3}\right) \delta 159.5,150.7,130.1,129.5,121.2,113.9$, 77.5, 77.2, 76.9, 76.0, 55.3, 37.3, 37.0, 16.1. HRMS (EI): $\mathrm{m} / \mathrm{z}[\mathrm{M}+\mathrm{Na}]^{+}$calcd for $\mathrm{C}_{13} \mathrm{H}_{14} \mathrm{~N}_{2} \mathrm{NaO}_{2}$ : 253.0947, found: 253.0948 .

\section{benzyl (3-(((4-methoxybenzyl)oxy)imino)cyclobutyl) carbonate 1e}

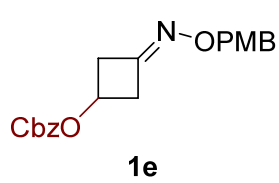

$1 \mathrm{e}$

1e was prepared from the corresponding cyclobutanone $(5.0 \mathrm{mmol})$ as white soild $(1.16 \mathrm{~g}, 65 \%)$. ${ }^{1} \mathrm{H}$ NMR $\left(400 \mathrm{MHz}, \mathrm{CDCl}_{3}\right) \delta 7.37-7.25(\mathrm{~m}, 7 \mathrm{H}), 6.88(\mathrm{~d}, J=8.63 \mathrm{~Hz}, 2 \mathrm{H}), 4.97(\mathrm{~s}, 2 \mathrm{H}), 4.45(\mathrm{~s}$, 2H), $4.23-4.14(\mathrm{~m}, 1 \mathrm{H}), 3.80(\mathrm{~s}, 3 \mathrm{H}), 3.23-3.08(\mathrm{~m}, 2 \mathrm{H}), 2.95-2.82(\mathrm{~m}, 2 \mathrm{H}) .{ }^{13} \mathrm{C}$ NMR $(100$ $\left.\mathrm{MHz}, \mathrm{CDCl}_{3}\right) \delta 159.4,152.7,137.5,130.1,129.9,128.5,128.0,113.8,77.4,77.1,76.8,75.5,71.0$, 67.3, 55.3, 40.1, 39.6. HRMS (EI): $\mathrm{m} / \mathrm{z}[\mathrm{M}+\mathrm{Na}]^{+}$calcd for $\mathrm{C}_{20} \mathrm{H}_{21} \mathrm{NNaO}_{5}: 378.1312$, found: 378.1310 .

\section{benzyl (3-(((4-methoxybenzyl)oxy)imino)cyclobutyl)carbamate 1f}

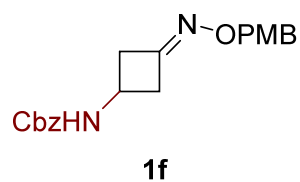

If was prepared from the corresponding cyclobutanone $(5.0 \mathrm{mmol})$ as white soild $(1.13 \mathrm{~g}, 64 \%)$. ${ }^{1} \mathrm{H}$ NMR $\left(400 \mathrm{MHz}, \mathrm{CDCl}_{3}\right) \delta 7.36-7.25(\mathrm{~m}, 5 \mathrm{H}), 7.27$ (d, J=5.02 Hz, 2H), $6.88(\mathrm{~d}, J=8.62 \mathrm{~Hz}$, $2 \mathrm{H}), 5.12-5.02(\mathrm{~m}, 3 \mathrm{H}), 4.97(\mathrm{~s}, 2 \mathrm{H}), 4.27(\mathrm{~s}, 1 \mathrm{H}), 3.80(\mathrm{~s}, 3 \mathrm{H}), 3.38-3.26(\mathrm{~m}, 2 \mathrm{H}), 2.81(\mathrm{t}, J=$ $22.83 \mathrm{~Hz}, 2 \mathrm{H}) .{ }^{13} \mathrm{C} \mathrm{NMR}\left(100 \mathrm{MHz}, \mathrm{CDCl}_{3}\right) \delta 159.4,155.6,152.6,136.2,129.9,128.6,128.6$, 128.3, 128.3, 128.2, 113.8, 77.4, 77.1, 76.8, 75.6, 66.9, 40.8, 40.5, 39.7. HRMS (EI): m/z [M $+\mathrm{Na}]^{+}$calcd for $\mathrm{C}_{20} \mathrm{H}_{22} \mathrm{~N}_{2} \mathrm{NaO}_{4}$ : 377.1472 , found: 377.1472 .

\section{3-phenylcyclobutan-1-one O-(4-methoxybenzyl) oxime 1g}

N-OPMB $1 \mathrm{~g}$ was prepared from the corresponding cyclobutanone $(5.0 \mathrm{mmol})$ as yellow oil $(900 \mathrm{mg}, 64 \%) .{ }^{1} \mathrm{H}$ NMR (400 MHz, $\left.\mathrm{CDCl}_{3}\right) \delta 7.32-7.20(\mathrm{~m}, 7 \mathrm{H}), 6.88(\mathrm{~d}, J=8.46 \mathrm{~Hz}, 2 \mathrm{H}), 5.01(\mathrm{~s}, 2 \mathrm{H}), 3.77(\mathrm{~s}, 3 \mathrm{H})$, $\mathrm{Ph} 1 \mathrm{~g} \quad 3.58-3.50(\mathrm{~m}, 1 \mathrm{H}), 3.41-3.29(\mathrm{~m}, 2 \mathrm{H}), 3.00(\mathrm{dd}, \mathrm{J}=13.00,3.17 \mathrm{~Hz}, 2 \mathrm{H}) .{ }^{13} \mathrm{C} \mathrm{NMR}(100 \mathrm{MHz}$,
$\left.\mathrm{CDCl}_{3}\right) \delta 159.4,155.8,144.2,130.2,130.0,128.7,126.6,126.5,113.9,77.5,77.2,76.9,75.6,55.3,39.7,39.0,32.9$. HRMS (EI): $\mathrm{m} / \mathrm{z}[\mathrm{M}+\mathrm{Na}]^{+}$calcd for $\mathrm{C}_{18} \mathrm{H}_{19} \mathrm{NNaO}_{2}: 304.1308$, found: 304.1311 .

\section{3-(naphthalen-2-yl)cyclobutan-1-one O-(4-methoxybenzyl) oxime $1 \mathrm{~h}$}

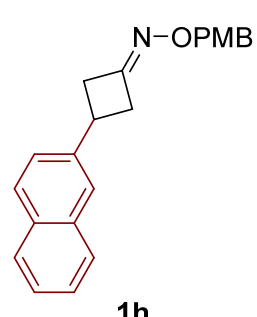

$1 \mathrm{~h}$

1h was prepared from the corresponding cyclobutanone $(5.0 \mathrm{mmol})$ as white soild $(1.16 \mathrm{~g}, 70 \%) .{ }^{1} \mathrm{H}$ NMR (400 MHz, $\left.\mathrm{CDCl}_{3}\right) \delta 7.79(\mathrm{t}, J=8.69 \mathrm{~Hz}, 3 \mathrm{H}), 7.65(\mathrm{~s}, 1 \mathrm{H}), 7.48-7.42(\mathrm{~m}, 2 \mathrm{H}), 7.35(\mathrm{t}, J=$ $9.17 \mathrm{~Hz}, 3 \mathrm{H}), 6.90(\mathrm{~d}, J=8.14 \mathrm{~Hz}, 2 \mathrm{H}), 5.03(\mathrm{~s}, 2 \mathrm{H}), 3.80(\mathrm{~s}, 3 \mathrm{H}), 3.77-3.69(\mathrm{~m}, 1 \mathrm{H}), 3.50-3.38$ $(\mathrm{m}, 2 \mathrm{H}), 3.11(\mathrm{dt}, J=14.63,7.32 \mathrm{~Hz}, 2 \mathrm{H}) .{ }^{13} \mathrm{C} \mathrm{NMR}\left(100 \mathrm{MHz}, \mathrm{CDCl}_{3}\right) \delta 159.3,155.7,141.3,133.2$, $132.1,130.0,129.9,128.4,127.6,126.2,125.6,124.8,124.6,113.7,77.3$, 77.0, 76.7 , 75.4, 55.2, 39.5, 38.8, 33.0. HRMS (EI): $\mathrm{m} / \mathrm{z}[\mathrm{M}+\mathrm{Na}]^{+}$calcd for $\mathrm{C}_{22} \mathrm{H}_{21} \mathrm{NNaO}_{2}: 354.1465$, found: 354.1466 . 


\section{methyl 6-(3-(((4-methoxybenzyl)oxy)imino)cyclobutyl)-2-naphthoate 1i}

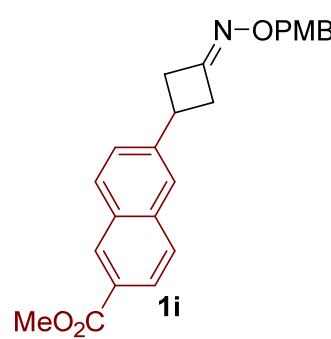

64\%). ${ }^{1} \mathrm{H}$ NMR (400 MHz, $\left.\mathrm{CDCl}_{3}\right) \delta 8.58(\mathrm{~s}, 1 \mathrm{H}), 8.06(\mathrm{~d}, J=8.41 \mathrm{~Hz}, 1 \mathrm{H}), 7.87$ (dd, $J=$ $38.42,8.56 \mathrm{~Hz}, 2 \mathrm{H}), 7.69$ (s, 1H), 7.39 (dd, $J=40.34,8.38 \mathrm{~Hz}, 3 \mathrm{H}), 6.90$ (d, $J=8.25 \mathrm{~Hz}, 2 \mathrm{H})$, $5.03(\mathrm{~s}, 2 \mathrm{H}), 3.98(\mathrm{~s}, 3 \mathrm{H}), 3.82(\mathrm{~s}, 3 \mathrm{H}), 3.79-3.73(\mathrm{~m}, 1 \mathrm{H}), 3.52-3.41(\mathrm{~m}, 2 \mathrm{H}), 3.16-3.08$ $(\mathrm{m}, 2 \mathrm{H}) .{ }^{13} \mathrm{C} \mathrm{NMR}\left(100 \mathrm{MHz}, \mathrm{CDCl}_{3}\right) \delta 167.2,159.4,155.2,144.1,135.5,131.3,130.8,130.1$, 129.9, 129.9, 127.9, 125.8, 125.7, 124.6, 113.8, 77.4, 76.7, 75.6, 55.3, 52.2, 39.4, 38.8, 33.2. HRMS (EI): $\mathrm{m} / \mathrm{z}[\mathrm{M}+\mathrm{Na}]^{+}$calcd for $\mathrm{C}_{24} \mathrm{H}_{23} \mathrm{NNaO}_{4}: 412.1519$, found: 412.1517 .

\section{3-methyl-3-phenylcyclobutan-1-one O-(4-methoxybenzyl) oxime 1j}

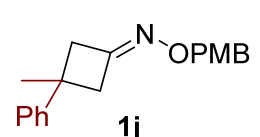

$1 \mathrm{j}$

1j was prepared from the corresponding cyclobutanone $(5.0 \mathrm{mmol})$ as yellow oil $(1.00 \mathrm{~g}, 68 \%)$. ${ }^{1} \mathrm{H}$ NMR (400 MHz, $\left.\mathrm{CDCl}_{3}\right) \delta 7.34-7.30(\mathrm{~m}, 4 \mathrm{H}), 7.22-7.18(\mathrm{~m}, 3 \mathrm{H}), 6.88(\mathrm{~d}, J=8.40 \mathrm{~Hz}, 2 \mathrm{H}), 5.01$ $(\mathrm{s}, 2 \mathrm{H}), 3.78(\mathrm{~s}, 3 \mathrm{H}), 3.23(\mathrm{t}, J=15.77 \mathrm{~Hz}, 2 \mathrm{H}), 2.99(\mathrm{dd}, J=34.02,17.55 \mathrm{~Hz}, 2 \mathrm{H}), 1.49(\mathrm{~s}, 3 \mathrm{H}) .{ }^{13} \mathrm{C}$ NMR $\left(100 \mathrm{MHz}, \mathrm{CDCl}_{3}\right) \delta 159.3,154.9,148.9,130.2,129.8,128.5,126.0,125.3,113.8,77.4,77.1,76.8,75.4,55.3$, 44.9, 44.3, 38.0, 31.0. HRMS (EI): $\mathrm{m} / \mathrm{z}[\mathrm{M}+\mathrm{Na}]^{+}$calcd for $\mathrm{C}_{19} \mathrm{H}_{21} \mathrm{NNaO}_{2}: 318.1465$, found: 318.1468 .

\section{tert-butyl 6-(((4-methoxybenzyl)oxy)imino)-2-azaspiro[3.3]heptane-2-carboxylate $1 \mathrm{k}$}

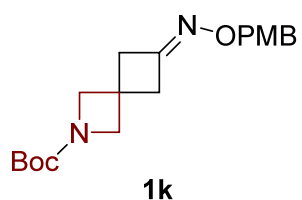

1k was prepared from the corresponding cyclobutanone $(5.0 \mathrm{mmol})$ as white soild $(1.21 \mathrm{~g}, 70 \%)$. ${ }^{1} \mathrm{H}$ NMR (400 MHz, $\left.\mathrm{CDCl}_{3}\right) \delta 7.28(\mathrm{~d}, J=8.45 \mathrm{~Hz}, 2 \mathrm{H}), 6.88(\mathrm{~d}, J=8.45 \mathrm{~Hz}, 2 \mathrm{H}), 4.96(\mathrm{~s}, 2 \mathrm{H})$, 3.97 (s, 4H), 3.79 (s, 3H), 3.07 (s, 4H), 1.43 (s, 9H). $\left.{ }^{13} \mathrm{C} \mathrm{NMR} \mathrm{(100} \mathrm{MHz,} \mathrm{CDCl}_{3}\right) \delta$ 159.4, 156.0, $152.8,129.9,129.9,113.8,79.7,77.5,77.1,76.8,75.6,55.2,42.9,42.3,31.8,28.4$, 22.0. HRMS (EI): $\mathrm{m} / \mathrm{z}[\mathrm{M}+\mathrm{Na}]^{+}$calcd for $\mathrm{C}_{19} \mathrm{H}_{26} \mathrm{~N}_{2} \mathrm{NaO}_{4}: 369.1785$, found: 369.1786 .

\section{tert-butyl 2-(((4-methoxybenzyl)oxy)imino)-7-azaspiro[3.5]nonane-7-carboxylate 11}

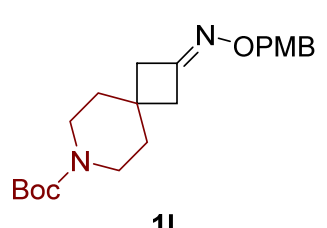

11

1l was prepared from the corresponding cyclobutanone $(5.0 \mathrm{mmol})$ as white soild $(1.27 \mathrm{~g}, 68 \%)$. ${ }^{1} \mathrm{H}$ NMR (400 MHz, $\left.\mathrm{CDCl}_{3}\right) \delta 7.30(\mathrm{~d}, J=8.60 \mathrm{~Hz}, 2 \mathrm{H}), 6.88(\mathrm{~d}, J=8.63 \mathrm{~Hz}, 2 \mathrm{H}), 4.98(\mathrm{~s}, 2 \mathrm{H})$, $3.80(\mathrm{~s}, 3 \mathrm{H}), 3.34(\mathrm{~m}, 4 \mathrm{H}), 2.63(\mathrm{~d}, J=3.46 \mathrm{~Hz}, 4 \mathrm{H}), 1.58(\mathrm{t}, J=5.50 \mathrm{~Hz}, 4 \mathrm{H}), 1.45(\mathrm{~s}, 9 \mathrm{H}) .{ }^{13} \mathrm{C}$ NMR $\left(100 \mathrm{MHz}, \mathrm{CDCl}_{3}\right) \delta 159.3,154.8,154.7,130.0,129.8,113.8,79.5,77.4,77.0,76.7,75.5$, 55.3, 41.8, 41.2, 36.4, 33.2, 28.4. HRMS (EI): $\mathrm{m} / \mathrm{z}[\mathrm{M}+\mathrm{Na}]^{+}$calcd for $\mathrm{C}_{21} \mathrm{H}_{30} \mathrm{~N}_{2} \mathrm{NaO}_{4}$ :

397.2098, found: 397.2103 .

\section{3-(((4-methoxybenzyl)oxy)imino)-2,2,4,4-tetramethylcyclobutan-1-one 1m}

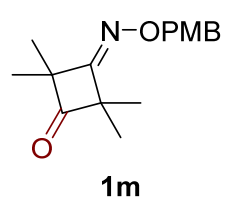

$1 \mathrm{~m}$ was prepared from the corresponding cyclobutanone $(5.0 \mathrm{mmol})$ as white soild $(908 \mathrm{mg}, 66 \%) .{ }^{1} \mathrm{H}$ NMR (400 MHz, $\left.\mathrm{CDCl}_{3}\right) \delta 7.29(\mathrm{~d}, J=8.33 \mathrm{~Hz}, 2 \mathrm{H}), 6.89$ (d, J=8.56 Hz, 2H), 5.02 (s, 2H), 3.82 (s, $3 \mathrm{H}), 1.37$ (d, 12H). ${ }^{13} \mathrm{C}$ NMR $\left(100 \mathrm{MHz}, \mathrm{CDCl}_{3}\right) \delta 216.8,164.9,159.3,129.8,129.6,113.7,77.4,77.0$, 76.7, 75.8, 64.5, 61.9, 55.3, 21.6, 19.8. HRMS (EI): $\mathrm{m} / \mathrm{z}[\mathrm{M}+\mathrm{H}]^{+}$calcd for $\mathrm{C}_{16} \mathrm{H}_{22} \mathrm{NO}_{3}: 276.1594$, found: 276.1592 .

\section{oxetan-3-one O-(4-methoxybenzyl) oxime 1n}

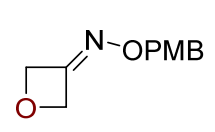

$1 \mathrm{n}$

1n was prepared from the corresponding cyclobutanone $(5.0 \mathrm{mmol})$ as white soild $(1.04 \mathrm{~g}, 80 \%) .{ }^{1} \mathrm{H}$ NMR (400 MHz, $\left.\mathrm{CDCl}_{3}\right) \delta 7.27(\mathrm{~d}, J=8.36 \mathrm{~Hz}, 2 \mathrm{H}), 6.88(\mathrm{~d}, J=8.42 \mathrm{~Hz}, 2 \mathrm{H}), 4.97$ (s, 2H), 4.04 (d, $J$ $=20.74 \mathrm{~Hz}, 4 \mathrm{H}), 3.80(\mathrm{~s}, 3 \mathrm{H}) .{ }^{13} \mathrm{C} \mathrm{NMR}\left(100 \mathrm{MHz}, \mathrm{CDCl}_{3}\right) \delta 159.5,151.6,130.0,129.5,113.9$, 77.5, 77.2, 76.8, 75.9, 55.3, 36.2, 36.0. HRMS (EI): $\mathrm{m} / \mathrm{z}[\mathrm{M}+\mathrm{Na}]^{+}$calcd for $\mathrm{C}_{11} \mathrm{H}_{13} \mathrm{NNaO}_{3}: 230.0788$, found:

230.0788 


\section{(1S,5R,E)-bicyclo[3.2.0]hept-2-en-6-one O-(4-methoxybenzyl) oxime 10}

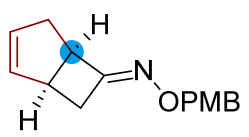

10

10 (mixture, $E / Z=2: 1)$ was prepared from the corresponding cyclobutanone $(5.0 \mathrm{mmol}$ ) as white soild $(827 \mathrm{mg}, 68 \%) .{ }^{1} \mathrm{H}$ NMR $\left(400 \mathrm{MHz}, \mathrm{CDCl}_{3}\right) \delta 7.30-7.25(\mathrm{~m}, 4 \mathrm{H}), 6.89-6.85(\mathrm{~m}, 3 \mathrm{H}), 5.76(\mathrm{~d}$, $J=3.26 \mathrm{~Hz}, 3 \mathrm{H}), 4.96(\mathrm{~s}, 3 \mathrm{H}), 3.79(\mathrm{~s}, 1 \mathrm{H}), 3.79(\mathrm{~s}, 3 \mathrm{H}), 3.75-3.70(\mathrm{~m}, 2 \mathrm{H}), 3.35-3.28(\mathrm{~m}, 2 \mathrm{H})$, $3.11-2.99(\mathrm{~m}, 2 \mathrm{H}), 2.65-2.47(\mathrm{~m}, 5 \mathrm{H}) .{ }^{13} \mathrm{C} \mathrm{NMR}\left(100 \mathrm{MHz}, \mathrm{CDCl}_{3}\right) \delta 162.8,159.3,132.7,132.0$, 131.9, 131.9, 130.3, 130.1, 129.8, 129.7, 113.7, 113.7, 77.4, 77.0, 76.7, 75.4, 75.4, 55.3, 46.5, 46.3, 41.1, 40.0, 38.8, 38.4, 37.8, 35.5. HRMS (EI): $\mathrm{m} / \mathrm{z}[\mathrm{M}+\mathrm{Na}]^{+}$calcd for $\mathrm{C}_{15} \mathrm{H}_{17} \mathrm{NNaO}_{2}: 266.1151$, found: 266.1151 .

\section{(E)-2,2-dimethylcyclopentan-1-one O-(4-methoxybenzyl) oxime 1p}

PMBO 1p was prepared from the corresponding cyclobutanone (5.0 mmol) as colorless oil (794 $\mathrm{mg}, 64 \%) .{ }^{1} \mathrm{H}$ NMR<smiles>CC1(C)CCCC1=N</smiles>
$\left(400 \mathrm{MHz}, \mathrm{CDCl}_{3}\right) \delta 7.30$ (d, $\left.J=8.68 \mathrm{~Hz}, 2 \mathrm{H}\right), 6.87$ (d, $\left.J=8.68 \mathrm{~Hz}, 2 \mathrm{H}\right), 4.99$ (s, 2H), 3.80 (s, 3H), 2.47 (t, $J$ $=7.49 \mathrm{~Hz}, 2 \mathrm{H}), 1.72-1.67(\mathrm{~m}, 2 \mathrm{H}), 1.59-1.56(\mathrm{~m}, 2 \mathrm{H}), 1.14(\mathrm{~s}, 6 \mathrm{H}) .{ }^{13} \mathrm{C} \mathrm{NMR}\left(100 \mathrm{MHz}, \mathrm{CDCl}_{3}\right) \delta 171.3$, $159.1,130.5,129.8,113.6,77.4,77.0,76.7,75.2,55.3,42.0,41.1,27.6,26.6,20.9$. HRMS (EI): $\mathrm{m} / \mathrm{z}$ [M + $\mathrm{Na}]^{+}$calcd for $\mathrm{C}_{15} \mathrm{H}_{21} \mathrm{NNaO}_{2}: 270.1465$, found: 270.1462 .

(E)-2-methyldihydrofuran-3(2H)-one O-(4-methoxybenzyl) oxime 1q

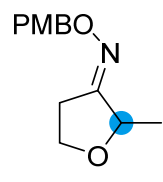

$1 \mathbf{q}$

1q was prepared from the corresponding cyclobutanone $(5.0 \mathrm{mmol})$ as colorless oil $(1.00 \mathrm{~g}, 85 \%) .{ }^{1} \mathrm{H}$ NMR $\left(400 \mathrm{MHz}, \mathrm{CDCl}_{3}\right) \delta 7.30$ (d, $\left.J=8.08 \mathrm{~Hz}, 2 \mathrm{H}\right), 6.88(\mathrm{~d}, J=8.22 \mathrm{~Hz}, 2 \mathrm{H}), 5.02(\mathrm{~s}, 2 \mathrm{H}), 4.28-4.33$ (q, $J=$ $6.09 \mathrm{~Hz}, 1 \mathrm{H}), 4.12-4.06(\mathrm{~m}, 1 \mathrm{H}), 3.83-3.77(\mathrm{~m}, 4 \mathrm{H}), 2.75-2.60(\mathrm{~m}, 2 \mathrm{H}), 1.37$ (d, $J=6.32 \mathrm{~Hz}, 3 \mathrm{H}) .{ }^{13} \mathrm{C}$ NMR $\left(100 \mathrm{MHz}, \mathrm{CDCl}_{3}\right) \delta 163.9,159.3,129.8,113.7,77.3,77.0,76.7,75.7,74.3,65.9,55.2,28.5,18.3$. HRMS (EI): $\mathrm{m} / \mathrm{z}[\mathrm{M}+\mathrm{H}]^{+}$calcd for $\mathrm{C}_{13} \mathrm{H}_{18} \mathrm{NO}_{3}: 236.1281$, found: 236.1280 .

\section{Detailed Optimization of Reaction Conditions and Control Experiments}

\subsection{Optimization of Reaction Conditions}

\section{Table S1. Screen of Photocatalysts under Blue LEDs}

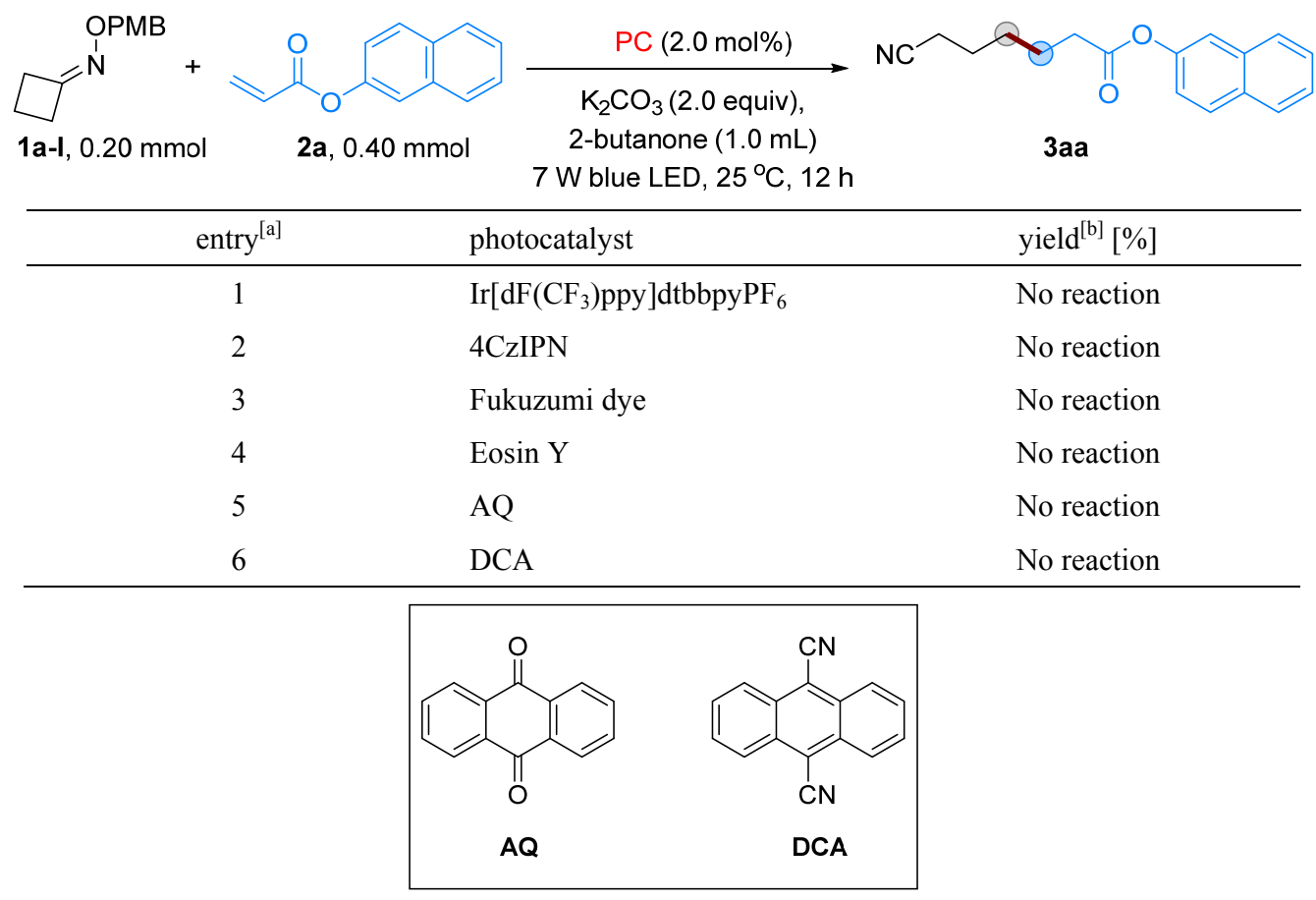


[a] Unless otherwise noted, reactions were carried out with 1a-I (41.06 mg, $0.20 \mathrm{mmol}), 2 \mathrm{a}(79.28 \mathrm{mg}, 0.40 \mathrm{mmol}), \mathrm{PC}(2 \mathrm{~mol} \%)$ and $\mathrm{K}_{2} \mathrm{CO}_{3}$ (55.28 mg, 2.0 equiv) in 2-butanone $(1.0 \mathrm{~mL})$ at $\mathrm{rt}$ under $7 \mathrm{~W}$ blue LEDs [b] Determined by isolated yield.

\section{Table S2. Screen of Organic Photocatalysts under 370 nm LED}

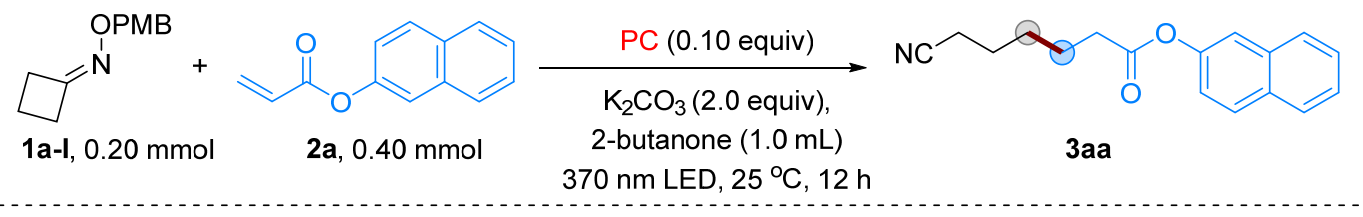<smiles>Cc1ccc2c(c1)C(=O)c1ccccc1C2=O</smiles>

$44 \%$ yield $^{[b]}$<smiles>O=C1c2ccccc2-c2ccccc21</smiles>

No reaction<smiles>Cc1ccc2c(c1)C(=O)c1ccccc1C2=O</smiles>

$31 \%$ yield $^{[b]}$<smiles>O=C(c1ccccc1)c1ccccn1</smiles>

No reaction<smiles>O=C1c2ccccc2C(=O)c2ccccc21</smiles>

$41 \%$ yield $^{[b]}$<smiles>O=C(c1ccccc1)c1ccccc1</smiles>

Not the desired point<smiles>O=C1c2ccccc2C(=O)c2c(Cl)cccc21</smiles>

$31 \%$ yield $^{[b]}$<smiles>N#CC1=C(C#N)C(=O)C(Cl)=C(Cl)C1=O</smiles>

No reaction<smiles></smiles><smiles>O=c1c2ccccc2sc2ccccc12</smiles>

No reaction

[a] Unless otherwise noted, reactions were carried out with 1a-I (41.06 mg, $0.20 \mathrm{mmol}), 2 \mathrm{a}(79.28 \mathrm{mg}, 0.40 \mathrm{mmol}), \mathrm{PC}(0.1 \mathrm{equiv})$ and $\mathrm{K}_{2} \mathrm{CO}_{3}(55.28 \mathrm{mg}, 2.0$ equiv) in 2-butanone $(1.0 \mathrm{~mL})$ at $\mathrm{rt}$ under $370 \mathrm{~nm}$ LED. [b] Determined by isolated yield.

As shown in Table S2, among all the photocatalysts tested, 2-Chloroanthraquinone gave the best results in terms of yield (44\% yield), and was thus selected for further optimization studies.

\section{Table S3. Screen of Ratio of 1a-I and 2a}

2-Chloroanthraquinone

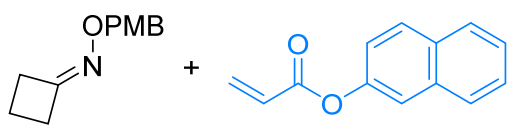

$1 \mathrm{a}-\mathrm{I}, 0.20 \mathrm{mmol}$

2a, X mmol ( 0.10 equiv)
$\mathrm{K}_{2} \mathrm{CO}_{3}(2.0$ equiv),

2-butanone (1.0 mL) $370 \mathrm{~nm}$ LED, $25^{\circ} \mathrm{C}, 12 \mathrm{~h}$

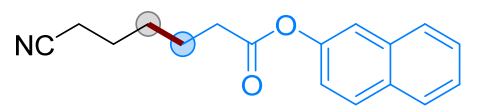

$3 a a$

\begin{tabular}{ccc}
\hline entry $^{[\mathrm{a}]}$ & ratio of 1a-I to $\mathbf{2 a}$ & yield $^{[\mathrm{b}]}[\%]$ \\
\hline 1 & $3: 1$ & 33 \\
2 & $2: 1$ & 42 \\
3 & $1: 1$ & 32 \\
4 & $1: 2$ & 44 \\
5 & $1: 3$ & 29 \\
\hline
\end{tabular}

[a] Unless otherwise noted, reactions were carried out with 1a-I (41.06 mg, $0.20 \mathrm{mmol}), 2 \mathrm{a}$ (x mmol), 2-chloroanthraquinone (24.27 $\mathrm{mg}, 0.10$ equiv) and $\mathrm{K}_{2} \mathrm{CO}_{3}(55.28 \mathrm{mg}, 2.0$ equiv) in 2-butanone $(1.0 \mathrm{~mL})$ at $\mathrm{rt}$ under $370 \mathrm{~nm} \mathrm{LED}$. [b] Determined by isolated yield. As shown in Table S3, among all the photocatalysts tested, a 1:2 ratio gave the best results in terms of yield (44\% yield), and was thus selected for further optimization studies. 
Table S4. Screen of Bases

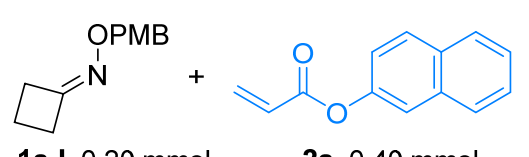

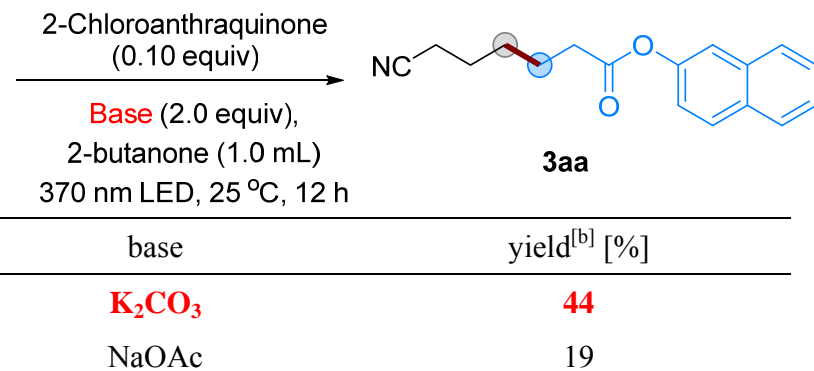

1a-I, $0.20 \mathrm{mmo}$

2a, $0.40 \mathrm{mmol}$

$\mathrm{Cs}_{2} \mathrm{CO}_{3}$

No reaction

3

$\mathrm{Li}_{2} \mathrm{CO}_{3}$

13

5

$\mathrm{Na}_{2} \mathrm{CO}_{3}$

32

6

$\mathrm{K}_{3} \mathrm{PO}_{4}$

14

7

$\mathrm{K}_{2} \mathrm{HPO}_{4}$

22

[a] Unless otherwise noted, reactions were carried out with 1a-I (41.06 mg, $0.20 \mathrm{mmol}), 2 \mathrm{2a}(79.28 \mathrm{mg}, 0.40 \mathrm{mmol})$, 2-chloroanthraquinone (24.27 mg, 0.10 equiv) and base (2.0 equiv) in 2-butanone (1.0 mL) at rt under $370 \mathrm{~nm}$ LED. [b] Determined by isolated yield.

As shown in Table S4, among the bases tested, $\mathrm{k}_{2} \mathrm{CO}_{3}$ gave the best results (44\% yield), and was thus selected for further studies.

Table S5. Screen of Solvents

\begin{tabular}{|c|c|c|c|}
\hline$\underbrace{\text { OPMB }}_{1 \mathrm{a}-\mathrm{I}, 0.20 \mathrm{mmol}} \mathrm{I}^{\mathrm{N}}+$ & $2 \mathrm{a}, 0.40 \mathrm{mmol}$ & $\begin{array}{c}\underset{2-C h l o r o a n t h r a q u i n o n e}{(0.10 \text { equiv })} \\
\underset{\mathrm{K}_{2} \mathrm{CO}_{3}(2.0 \text { equiv }),}{\text { solvent }(1.0 \mathrm{~mL})} \\
370 \mathrm{~nm} \mathrm{LED}, 25^{\circ} \mathrm{C}, 12 \mathrm{~h}\end{array}$ & 3aa \\
\hline & $e{ }^{[a]}$ & solvent & $\operatorname{yield}^{[\mathrm{b}]}[\%]$ \\
\hline & 1 & $\mathrm{CH}_{3} \mathrm{CN}$ & 27 \\
\hline & 2 & $\mathrm{CH}_{2} \mathrm{Cl}_{2}$ & 20 \\
\hline & 3 & DCE & 13 \\
\hline & 4 & toluene & 21 \\
\hline & 5 & DMSO & No reaction \\
\hline & 6 & DMF & 19 \\
\hline & 7 & $\mathrm{CH}_{3} \mathrm{OH}$ & No reaction \\
\hline & 8 & THF & 21 \\
\hline & 9 & 4-methyl-2-pentanone & 15 \\
\hline & 10 & cyclohexanone & 43 \\
\hline & 11 & 2-butanone & 44 \\
\hline
\end{tabular}

[a] Unless otherwise noted, reactions were carried out with 1a-I (41.06 mg, $0.20 \mathrm{mmol}), 2 \mathrm{2a}(79.28 \mathrm{mg}, 0.40 \mathrm{mmol})$, 2-chloroanthraquinone (24.27 mg, 0.10 equiv) and $\mathrm{K}_{2} \mathrm{CO}_{3}(55.28 \mathrm{mg}, 2.0$ equiv) in solvent (1.0 mL) at $\mathrm{rt}$ under $370 \mathrm{~nm} \mathrm{LED}$. [b] Determined by isolated yield.

As show in Table S5, among all the solvents tested, 2-butanone gave the best results in terms of yield (44\% yield), and was thus selected for further optimization studies. 


\section{Table S6. Screen of Concentration}

\begin{tabular}{|c|c|c|c|}
\hline 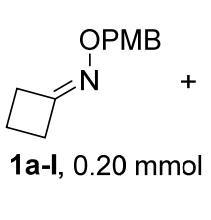 & 2a, $0.40 \mathrm{mmol}$ & $\begin{array}{c}\underset{\text { 2-Chloroanthraquinone }}{\text { (0.10 equiv })} \\
\mathrm{K}_{2} \mathrm{CO}_{3}(2.0 \text { equiv }), \\
\text { 2-butanone }(x \mathrm{~mL}) \\
370 \mathrm{~nm} \mathrm{LED}, 25^{\circ} \mathrm{C}, 12 \mathrm{~h}\end{array}$ & $\prod_{0}$ \\
\hline & entry $^{[a]}$ & concentration $(\mathrm{x} \mathrm{mL})$ & yield $^{[b]}[\%]$ \\
\hline & 1 & 1 & 44 \\
\hline & 2 & 2 & 43 \\
\hline & 3 & 4 & 47 \\
\hline & 4 & 6 & 35 \\
\hline
\end{tabular}

[a] Unless otherwise noted, reactions were carried out with 1a-I (41.06 mg, $0.20 \mathrm{mmol}), 2 \mathrm{2a}(79.28 \mathrm{mg}, 0.40 \mathrm{mmol})$, 2-chloroanthraquinone (24.27 mg, 0.10 equiv) and $\mathrm{K}_{2} \mathrm{CO}_{3}(55.28 \mathrm{mg}, 2.0$ equiv) in solvent ( $\mathrm{x} \mathrm{mL})$ at $\mathrm{rt}$ under $370 \mathrm{~nm}$ LED. [b] Determined by isolated yield.

As shown in Table S6, among the concentration tested, 2-butanone (4 mL) gave the best result in terms of yield (47\% yield) and the optimized reaction condition was confirmed.

\section{Table S7. Screen of Equivalent of PC}

\begin{tabular}{|c|c|c|c|}
\hline$\underbrace{\text { OPMB }}_{1 \mathrm{a}-\mathrm{I}, 0.20 \mathrm{mmol}} \mathrm{I}^{\mathrm{N}}+$ & $2 \mathrm{a}, 0.40 \mathrm{mmol}$ & $\begin{array}{c}\text { 2-Chloroanthraquinone } \\
\text { (x equiv) }\end{array}$ & 3aа \\
\hline & entry $^{[a]}$ & equivalent & yield $^{[b]}[\%]$ \\
\hline & 1 & 0.1 & 43 \\
\hline & 2 & 0.2 & 47 (59\% after $24 \mathrm{~h})$ \\
\hline & 3 & 0.5 & 67(recovery of PC: 88\%) \\
\hline & 4 & 1.0 & 54 \\
\hline
\end{tabular}

[a] Unless otherwise noted, reactions were carried out with 1a-I (41.06 mg, $0.20 \mathrm{mmol}), 2 \mathrm{2a}(79.28 \mathrm{mg}, 0.40 \mathrm{mmol})$, 2-chloroanthraquinone (X equiv) and $\mathrm{K}_{2} \mathrm{CO}_{3}(55.28 \mathrm{mg}, 2.0$ equiv) in 2-butanone (4.0 mL) at $\mathrm{rt}$ under $370 \mathrm{~nm}$ LED. [b] Determined by isolated yield.

As shown in Table S7, among the concentration tested, 2-chloroanthraquinone ( $24.27 \mathrm{mg}, 0.50$ equiv) gave the best result in terms of yield (67\% yield) and the optimized reaction condition was confirmed.

\section{Table S8. Screen of Protecting Group}

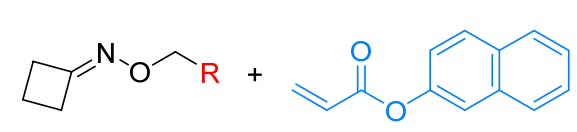

1a, $0.20 \mathrm{mmol}$

2a, $0.40 \mathrm{mmol}$

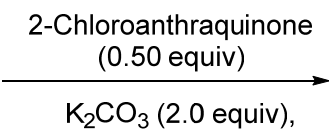

2-butanone $(4.0 \mathrm{~mL})$ $370 \mathrm{~nm}$ LED, $25^{\circ} \mathrm{C}, 12 \mathrm{~h}$

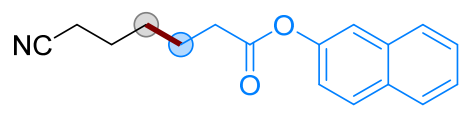

3aa

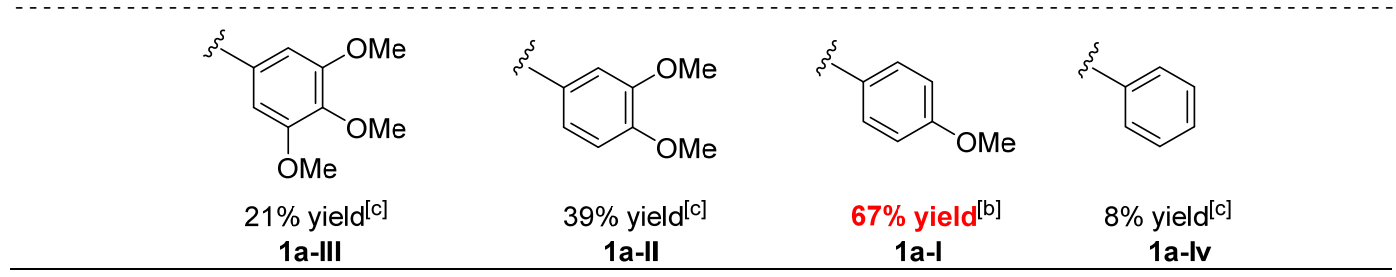


[a] Unless otherwise noted, reactions were carried out with 1a (41.06 mg, $0.20 \mathrm{mmol}), 2 \mathrm{2a}(79.28 \mathrm{mg}, 0.40 \mathrm{mmol})$, 2-chloroanthraquinone (24.27 mg, 0.50 equiv) and $\mathrm{K}_{2} \mathrm{CO}_{3}(55.28 \mathrm{mg}, 2.0$ equiv) in 2-butanone (4.0 mL) at $\mathrm{rt}$ under $370 \mathrm{~nm} \mathrm{LED}$. [b] Isolated yield. [c] ${ }^{1} \mathrm{H}$ NMR yield with 1,3,5-trimethoxybenzene as an internal standard.

As shown in Table S8, among the concentration tested, $p$ - $\mathrm{MeOC}_{4} \mathrm{H}_{4}$ group gave the best result in terms of yield $(67 \%$ yield) and the optimized reaction condition was confirmed.

\subsection{Control Experiments for Synthesis of 3aa}

\section{Table S9. Control Experiments}

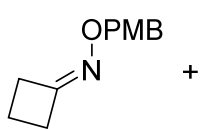

1a-I, $0.20 \mathrm{mmol}$

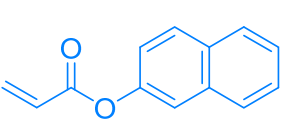

2a, $0.40 \mathrm{mmol}$

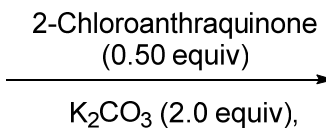

2-butanone $(4.0 \mathrm{~mL})$ $370 \mathrm{~nm}$ LED, $25^{\circ} \mathrm{C}, 12 \mathrm{~h}$

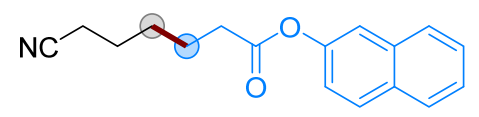

3aa

\begin{tabular}{ccccc}
\hline entry $^{a}$ & hv & photocatalyst & base & yield $^{b}(\%)$ \\
\hline 1 & - & + & + & No reaction \\
2 & + & - & + & No reaction \\
3 & + & + & - & 22 \\
4 & + & + & + & 67 \\
\hline
\end{tabular}

[a] Unless otherwise noted, reactions were carried out with 1a-I (41.06 mg, $0.20 \mathrm{mmol}), 2 \mathrm{2a}(79.28 \mathrm{mg}, 0.40 \mathrm{mmol})$, 2-chloroanthraquinone (24.27 mg, 0.50 equiv) and $\mathrm{K}_{2} \mathrm{CO}_{3}(55.28 \mathrm{mg}, 2.0$ equiv) in 2-butanone (4.0 mL) at $\mathrm{rt}$ under $370 \mathrm{~nm} \mathrm{LED}$. [b] Determined by isolated yield.

The results of Table $S 9$ reveal that reaction is indeed a photocatalytic process.

\section{General Procedure and Spectral Data of Products}

\subsection{General Procedure for Synthesis of 3}

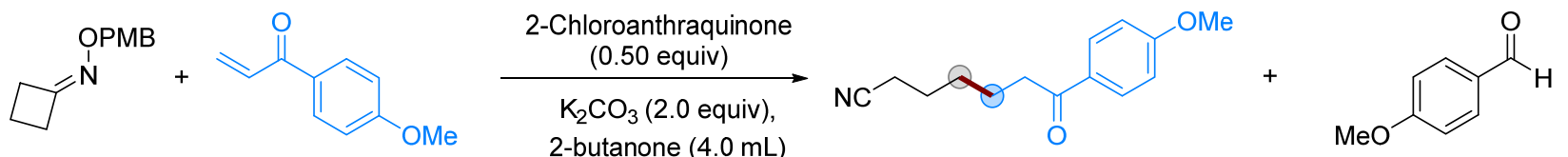

$1 \mathrm{a}-\mathrm{I}, 0.20 \mathrm{mmol}$

2b, $0.40 \mathrm{mmol}$

$370 \mathrm{~nm}$ LED, $25^{\circ} \mathrm{C}, 12 \mathrm{~h}$

3ab, $32.84 \mathrm{mg}, 70 \%$ yield

6, $25.6 \mathrm{mg}, 94 \%$ yield

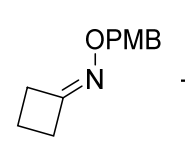

$1 \mathrm{a}-\mathrm{I}, 0.20 \mathrm{mmo}$

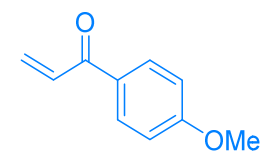

2b, $0.40 \mathrm{mmol}$

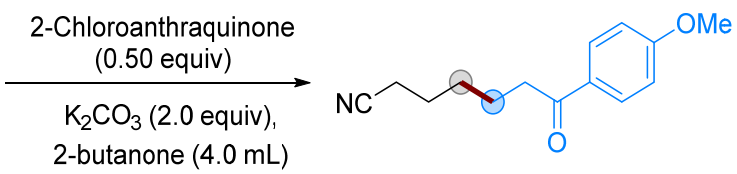

$390 \mathrm{~nm}$ LED, $25^{\circ} \mathrm{C}, 12 \mathrm{~h}$

$3 \mathrm{ab}, 32.38 \mathrm{mg}, 70 \%$ yield

In a dried sealed tube, 1a-I (41.06 mg, $0.20 \mathrm{mmol}), 2 \mathbf{b}(64.88 \mathrm{mg}, 0.40 \mathrm{mmol})$, 2-chloroanthraquinone $(24.27 \mathrm{mg}, 0.50$ equiv) and $\mathrm{K}_{2} \mathrm{CO}_{3}(55.28 \mathrm{mg}, 2.0$ equiv) were dissolved in 2-butanone (4.0 mL) under a Ar atmosphere. Then, the resulting mixture was degassed via "freeze-pump-thaw" procedure (3 times). After that, the solution was stirred at a distance of $\sim 5 \mathrm{~cm}$ from a $370 \mathrm{~nm}$ LED (or $390 \mathrm{~nm}$ LED) at room temperature about $12 \mathrm{~h}$ until the reaction was completed as monitored by TLC analysis. The crude product was purified by flash chromatography on silica gel 
(petroleum ether/ethylacetate 5:1) directly to give the aldehyde in 94\% yield and the desired product 3ab in $71 \%$ yield (or $70 \%$ yield) as a colorless oil.

\subsection{Spectral Data of Products}

\section{7-(4-methoxyphenyl)-7-oxoheptanenitrile 3ab}

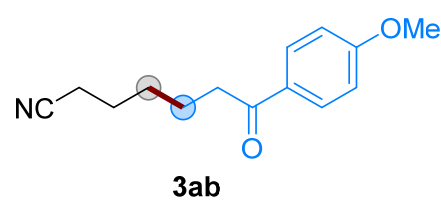

$46.2 \mathrm{mg}$, colorless oil, $71 \%$ yield. ${ }^{1} \mathrm{H}$ NMR $\left(400 \mathrm{MHz}, \mathrm{CDCl}_{3}\right) \delta(\mathrm{ppm}) 7.93(\mathrm{~d}, J=$ $8.86 \mathrm{~Hz}, 2 \mathrm{H}), 6.93(\mathrm{~d}, J=8.87 \mathrm{~Hz}, 2 \mathrm{H}), 3.87(\mathrm{~s}, 3 \mathrm{H}), 2.95(\mathrm{t}, J=7.22 \mathrm{~Hz}, 2 \mathrm{H}), 2.37(\mathrm{t}$, $J=7.09 \mathrm{~Hz}, 2 \mathrm{H}), 1.78-1.68(\mathrm{~m}, 4 \mathrm{H}), 1.57-1.51(\mathrm{~m}, 2 \mathrm{H}) .{ }^{13} \mathrm{C}$ NMR $(100 \mathrm{MHz}$, $\left.\mathrm{CDCl}_{3}\right) \delta(\mathrm{ppm}) \delta 198.4,163.5,130.3,129.9,119.7,113.8,55.5,37.7,28.4,25.3,23.4$, 17.1. IR (in KBr): $3450,3129,2940,1673,1601,1257,1172 \mathrm{~cm}^{-1}$. HRMS (EI): $\mathrm{m} / \mathrm{z}[\mathrm{M}+\mathrm{Na}]^{+}$calcd for $\mathrm{C}_{14} \mathrm{H}_{17} \mathrm{NNaO}_{2}$ : 254.1151, found: 254.1150 .

\section{tert-butyl 2-(cyanomethyl)-6-(4-methoxyphenyl)-6-oxohexanoate 3bb}

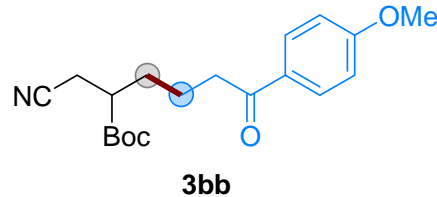

$47.7 \mathrm{mg}$, colorless oil, $72 \%$ yield. ${ }^{1} \mathrm{H}$ NMR $\left(400 \mathrm{MHz}, \mathrm{CDCl}_{3}\right) \delta(\mathrm{ppm}) 7.98(\mathrm{~d}, J=$ $8.80 \mathrm{~Hz}, 2 \mathrm{H}), 6.98(\mathrm{~d}, J=8.79 \mathrm{~Hz}, 2 \mathrm{H}), 3.92(\mathrm{~s}, 3 \mathrm{H}), 3.01$ (t, $J=5.35 \mathrm{~Hz}, 2 \mathrm{H}), 2.74-$ $2.58(\mathrm{~m}, 3 \mathrm{H}), 1.88-1.76(\mathrm{~m}, 4 \mathrm{H}), 1.53(\mathrm{~s}, 9 \mathrm{H}) .{ }^{13} \mathrm{C} \mathrm{NMR}\left(100 \mathrm{MHz}, \mathrm{CDCl}_{3}\right) \delta(\mathrm{ppm})$ $197.8,171.7,163.5,130.3,129.8,117.9,113.8,113.7,82.2,77.4,55.5,42.3,37.5,31.1$, 28.0, 21.2, 19.5. IR (in KBr): $3437,3129,2975,1726,1602,1162 \mathrm{~cm}^{-1}$. HRMS (EI): $\mathrm{m} / \mathrm{z}[\mathrm{M}+\mathrm{Na}]^{+}$calcd for $\mathrm{C}_{19} \mathrm{H}_{25} \mathrm{NnaO}_{4}$ : 354.1676, found: 354.1673 .

\section{methyl 2-(cyanomethyl)-6-(4-methoxyphenyl)-6-oxohexanoate 3cb}

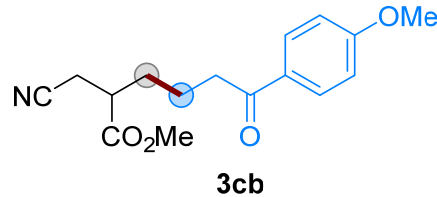

$43.1 \mathrm{mg}$, colorless oil, $74 \%$ yield. ${ }^{1} \mathrm{H}$ NMR $\left(400 \mathrm{MHz}, \mathrm{CDCl}_{3}\right) \delta(\mathrm{ppm}) 7.93(\mathrm{~d}, J=$ $8.91 \mathrm{~Hz}, 2 \mathrm{H}), 6.94$ (d, J=8.89 Hz, 2H), 3.87 (s, 3H), 3.75 (s, 3H), $2.99-2.59$ (m, 5H), $1.85-1.76(\mathrm{~m}, 4 \mathrm{H}) .{ }^{13} \mathrm{C}$ NMR $\left(100 \mathrm{MHz}, \mathrm{CDCl}_{3}\right) \delta(\mathrm{ppm}) 197.7,172.9,163.5,130.2$, 129.7, 117.7, 113.7, 55.4, 52.4, 41.4, 37.4, 30.8, 21.0, 19.2. IR (in KBr): 3451, 3129, 2953, 1736, 1673, $1173 \mathrm{~cm}^{-1}$. HRMS (EI): $\mathrm{m} / \mathrm{z}[\mathrm{M}+\mathrm{Na}]^{+}$calcd for $\mathrm{C}_{16} \mathrm{H}_{19} \mathrm{NNaO}_{4}: 312.1206$, found: 312.1210 .

\section{2-(4-(4-methoxyphenyl)-4-oxobutyl)succinonitrile 3db}

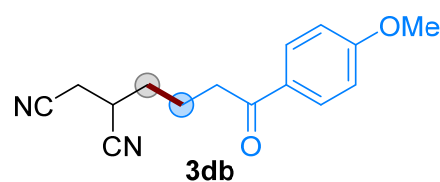

$3 \mathrm{db}$

$37.6 \mathrm{mg}$, colorless oil, 73\% yield. ${ }^{1} \mathrm{H}$ NMR $\left(400 \mathrm{MHz}, \mathrm{CDCl}_{3}\right) \delta(\mathrm{ppm}) 7.93(\mathrm{~d}, J=$ $8.91 \mathrm{~Hz}, 2 \mathrm{H}), 6.95(\mathrm{~d}, J=8.91 \mathrm{~Hz}, 2 \mathrm{H}), 3.88(\mathrm{~s}, 3 \mathrm{H}), 3.03(\mathrm{dt}, J=14.20,6.54 \mathrm{~Hz}, 3 \mathrm{H})$, $2.78(\mathrm{~d}, J=6.50 \mathrm{~Hz}, 2 \mathrm{H}), 2.05-1.85(\mathrm{~m}, 4 \mathrm{H}) .{ }^{13} \mathrm{C} \mathrm{NMR}\left(100 \mathrm{MHz}, \mathrm{CDCl}_{3}\right) \delta(\mathrm{ppm}) \delta$ $197.2,163.6,130.2,129.5,118.8,115.6,113.8,55.5,36.7,30.9,28.4,21.0,20.9$. IR (in KBr): $3418,3133,1751,1395,1285,1145 \mathrm{~cm}^{-1}$. HRMS (EI): $\mathrm{m} / \mathrm{z}[\mathrm{M}+\mathrm{Na}]^{+}$calcd for $\mathrm{C}_{15} \mathrm{H}_{16} \mathrm{~N}_{2} \mathrm{NaO}_{2}: 279.1104$, found: 279.1104

\section{benzyl (1-cyano-6-(4-methoxyphenyl)-6-oxohexan-2-yl) carbonate 3eb}

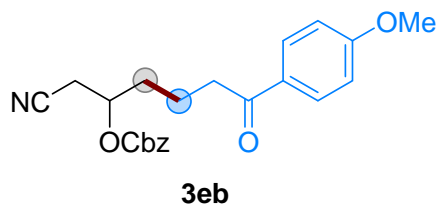

$51.4 \mathrm{mg}$, colorless oil, $67 \%$ yield. ${ }^{1} \mathrm{H}$ NMR $\left(400 \mathrm{MHz}, \mathrm{CDCl}_{3}\right) \delta(\mathrm{ppm}) 7.91(\mathrm{~d}, J=$ $8.92 \mathrm{~Hz}, 2 \mathrm{H}), 7.36-7.34(\mathrm{~m}, 5 \mathrm{H}), 6.93(\mathrm{~d}, J=8.90 \mathrm{~Hz}, 2 \mathrm{H}), 4.67-4.55(\mathrm{~m}, 2 \mathrm{H}), 3.87$ (s, 3H), $3.75-3.72(\mathrm{~m}, 1 \mathrm{H}), 2.93(\mathrm{t}, J=6.26 \mathrm{~Hz}, 2 \mathrm{H}), 2.59(\mathrm{t}, J=5.22 \mathrm{~Hz}, 2 \mathrm{H}), 1.87-$ $1.72(\mathrm{~m}, 4 \mathrm{H}) .{ }^{13} \mathrm{C}$ NMR $\left(100 \mathrm{MHz}, \mathrm{CDCl}_{3}\right) \delta(\mathrm{ppm}) 198.2,163.5,137.5,134.1,130.3$, 128.6, 128.0, 127.9, 117.6, 113.8, 74.3, 71.9, 55.5, 37.6, 33.8, 22.9, 19.8. IR (in KBr): 3447, 3131, 1670, 1398, 1171, $1090 \mathrm{~cm}^{-1}$. HRMS (EI): $\mathrm{m} / \mathrm{z}[\mathrm{M}+\mathrm{Na}]^{+}$calcd for $\mathrm{C}_{22} \mathrm{H}_{23} \mathrm{NNaO}_{5}: 404.1468$, found: 404.1468 . 
benzyl (1-cyano-6-(4-methoxyphenyl)-6-oxohexan-2-yl)carbamate 3fb<smiles></smiles>

$54.7 \mathrm{mg}$, colorless oil, $72 \%$ yield. ${ }^{1} \mathrm{H}$ NMR (400 MHz, $\left.\mathrm{CDCl}_{3}\right) \delta(\mathrm{ppm}) 7.92(\mathrm{~d}, 2 \mathrm{H})$, $7.37-7.32(\mathrm{~m}, 5 \mathrm{H}), 6.93(\mathrm{~d}, J=8.78 \mathrm{~Hz}, 2 \mathrm{H}), 5.19(\mathrm{~d}, J=7.52 \mathrm{~Hz}, 1 \mathrm{H}), 5.11(\mathrm{~s}, 2 \mathrm{H})$, $3.87(\mathrm{~s}, 3 \mathrm{H}), 2.99-2.59(\mathrm{~m}, 4 \mathrm{H}), 1.88-1.72(\mathrm{~m}, 4 \mathrm{H}) .{ }^{13} \mathrm{C} \mathrm{NMR}\left(100 \mathrm{MHz}, \mathrm{CDCl}_{3}\right) \delta$ (ppm) 198.0, 163.6, 155.8, 136.1, 130.3, 129.8, 128.6, 128.3, 128.1, 117.2, 113.8, 67.1, 55.5, 47.9, 37.1, 32.8, 23.8, 20.2. IR (in KBr): 3414, 1713, 1603, 1523, 1256, $1173 \mathrm{~cm}^{-1}$.

HRMS (EI): $\mathrm{m} / \mathrm{z}[\mathrm{M}+\mathrm{Na}]^{+}$calcd for $\mathrm{C}_{22} \mathrm{H}_{22} \mathrm{~N}_{2} \mathrm{NaO}_{4}: 403.1628$, found: 403.1629 .

\section{7-(4-methoxyphenyl)-7-oxo-3-phenylheptanenitrile 3gb}

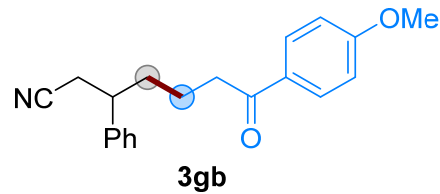

$41.9 \mathrm{mg}$, colorless oil, $68 \%$ yield. ${ }^{1} \mathrm{H}$ NMR $\left(400 \mathrm{MHz}, \mathrm{CDCl}_{3}\right) \delta(\mathrm{ppm}) 7.88(\mathrm{~d}, J=$ $8.75 \mathrm{~Hz}, 2 \mathrm{H}), 7.37-7.23$ (m, 5H), 6.91 (d, $J=8.74 \mathrm{~Hz}, 2 \mathrm{H}), 3.86$ (s, 3H), $3.04-2.87$ (m, 3H), $2.62(\mathrm{~d}, 2 \mathrm{H}), 1.92-1.62(\mathrm{~m}, 4 \mathrm{H}) .{ }^{13} \mathrm{C}$ NMR $\left(100 \mathrm{MHz}, \mathrm{CDCl}_{3}\right) \delta(\mathrm{ppm})$ 198.2, 163.4, 141.3, 130.2, 129.8, 128.9, 127.5, 127.2, 118.5, 113.7, 55.4, 42.2, 37.6, 34.4, 25.2, 22.0. IR (in KBr): $3430,3131,2934,1673,1402,1172 \mathrm{~cm}^{-1}$. HRMS (EI): $\mathrm{m} / \mathrm{z}[\mathrm{M}+\mathrm{H}]^{+}$calcd for $\mathrm{C}_{20} \mathrm{H}_{22} \mathrm{NO}_{2}$ : 308.1645, found: 308.1642 .

\section{7-(4-methoxyphenyl)-3-(naphthalen-2-yl)-7-oxoheptanenitrile 3hb}

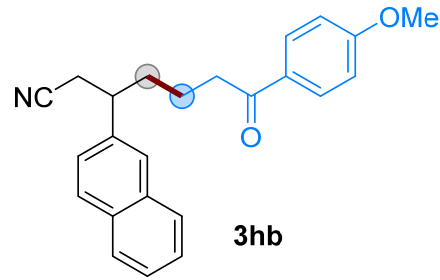

$47.9 \mathrm{mg}$, colorless oil, $67 \%$ yield. ${ }^{1} \mathrm{H}$ NMR $\left(400 \mathrm{MHz}, \mathrm{CDCl}_{3}\right) \delta(\mathrm{ppm}) 7.85-7.81(\mathrm{~m}$, $5 \mathrm{H}), 7.69(\mathrm{~s}, 1 \mathrm{H}), 7.52-7.46(\mathrm{~m}, 2 \mathrm{H}), 7.42-7.34(\mathrm{~m}, 1 \mathrm{H}), 6.86(\mathrm{~d}, J=7.99 \mathrm{~Hz}, 2 \mathrm{H})$, $3.83(\mathrm{~s}, 3 \mathrm{H}), 3.19-3.11(\mathrm{~m}, 1 \mathrm{H}), 2.95-2.82(\mathrm{~m}, 2 \mathrm{H}), 2.70(\mathrm{~d}, J=6.85 \mathrm{~Hz}, 2 \mathrm{H}), 2.04-$ $1.92(\mathrm{~m}, 2 \mathrm{H}), 1.73-1.59(\mathrm{~m}, 2 \mathrm{H}) .{ }^{13} \mathrm{C} \mathrm{NMR}\left(100 \mathrm{MHz}, \mathrm{CDCl}_{3}\right) \delta(\mathrm{ppm}) 198.2,163.3$, 138.6, 133.4, 132.7, 130.2, 129.7, 128.8, 127.7, 127.6, 126.4, 126.3, 125.9, 124.7, 118.5, 113.6, 55.4, 42.3, 37.6, 34.3, 25.1, 22.0. IR (in KBr): 3437, 3130, 2359, 1670, 1397,

$1171 \mathrm{~cm}^{-1}$. HRMS (EI): $\mathrm{m} / \mathrm{z}[\mathrm{M}+\mathrm{Na}]^{+}$calcd for $\mathrm{C}_{24} \mathrm{H}_{23} \mathrm{NNaO}_{2}: 380.1621$, found: 380.1619 .

\section{methyl 6-(1-cyano-6-(4-methoxyphenyl)-6-oxohexan-2-yl)-2-naphthoate 3ib}

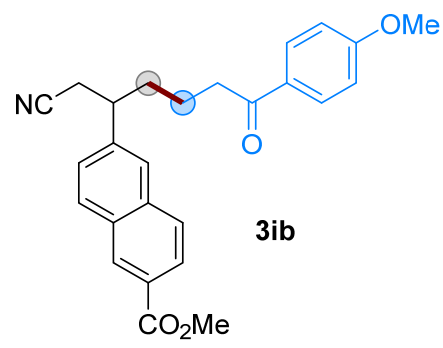

$68.1 \mathrm{mg}$, colorless oil, $85 \%$ yield. ${ }^{1} \mathrm{H}$ NMR $\left(400 \mathrm{MHz}, \mathrm{CDCl}_{3}\right) \delta(\mathrm{ppm}) 8.58(\mathrm{~s}, 1 \mathrm{H})$, 8.07 (dd, $J=8.55,1.60 \mathrm{~Hz}, 1 \mathrm{H}), 7.95(\mathrm{~d}, J=8.50 \mathrm{~Hz}, 1 \mathrm{H}), 7.86(\mathrm{dd}, J=8.62,3.95$ $\mathrm{Hz}, 3 \mathrm{H}), 7.74$ (s, 1H), 7.43 (d, $J=8.60 \mathrm{~Hz}, 1 \mathrm{H}), 6.88(\mathrm{~d}, J=8.49 \mathrm{~Hz}, 2 \mathrm{H}), 3.98$ (s, $3 \mathrm{H}), 3.84(\mathrm{~s}, 3 \mathrm{H}), 3.23-3.16(\mathrm{~m}, 1 \mathrm{H}), 2.99-2.85(\mathrm{~m}, 2 \mathrm{H}), 2.73(\mathrm{~d}, J=6.86 \mathrm{~Hz}, 2 \mathrm{H})$, $2.04-1.94(\mathrm{~m}, 2 \mathrm{H}), 1.76-1.61(\mathrm{~m}, 2 \mathrm{H}) .{ }^{13} \mathrm{C}$ NMR $\left(100 \mathrm{MHz}, \mathrm{CDCl}_{3}\right) \delta(\mathrm{ppm}) 198.1$, $167.2,163.5,141.4,135.6,131.9,130.8,130.4,130.2,129.8,128.1,127.5,126.4$, $125.8,125.7,118.3,113.7,55.5,52.3,42.6,37.6,34.3,25.1,22.1$. IR (in KBr): 3430 , 3130, 2947, 1717, 1401, $1174 \mathrm{~cm}^{-1}$. HRMS (EI): $\mathrm{m} / \mathrm{z}[\mathrm{M}+\mathrm{Na}]^{+}$calcd for $\mathrm{C}_{26} \mathrm{H}_{25} \mathrm{NNaO}_{4}: 438.1676$, found: 438,1674.

\section{7-(4-methoxyphenyl)-3-methyl-7-oxo-3-phenylheptanenitrile 3jb}

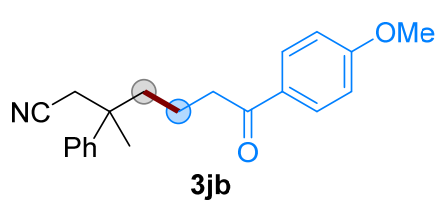

$43.1 \mathrm{mg}$, colorless oil, $67 \%$ yield. ${ }^{1} \mathrm{H}$ NMR $\left(400 \mathrm{MHz}, \mathrm{CDCl}_{3}\right) \delta(\mathrm{ppm}) 7.87(\mathrm{~d}, J=$ $8.88 \mathrm{~Hz}, 2 \mathrm{H}), 7.38-7.32(\mathrm{~m}, 5 \mathrm{H}), 6.91(\mathrm{~d}, J=8.89 \mathrm{~Hz}, 2 \mathrm{H}), 3.86(\mathrm{~s}, 3 \mathrm{H}), 2.86(\mathrm{t}, J$ $=7.13 \mathrm{~Hz}, 2 \mathrm{H}), 2.67(\mathrm{~s}, 2 \mathrm{H}), 2.00-1.78(\mathrm{~m}, 2 \mathrm{H}), 1.62-1.56(\mathrm{~m}, 4 \mathrm{H}), 1.50-1.41$ $(\mathrm{m}, 1 \mathrm{H}) .{ }^{13} \mathrm{C}$ NMR $\left(100 \mathrm{MHz}, \mathrm{CDCl}_{3}\right) \delta(\mathrm{ppm}) 198.2,163.5,143.9,130.3,130.0$, 128.8, 126.9, 125.8, 118.2, 113.8, 55.5, 41.2, 40.2, 38.2, 31.7, 25.0, 19.1. IR (in KBr): 3129, 1673, 1601, 1400, 1256, $1171 \mathrm{~cm}^{-1}$. HRMS (EI): $\mathrm{m} / \mathrm{z}[\mathrm{M}+\mathrm{Na}]^{+}$calcd for $\mathrm{C}_{21} \mathrm{H}_{23} \mathrm{NNaO}_{2}: 344.1621$, found: 344.1619 .

\section{tert-butyl 3-(cyanomethyl)-3-(4-(4-methoxyphenyl)-4-oxobutyl)azetidine-1 carboxylate 3kb}

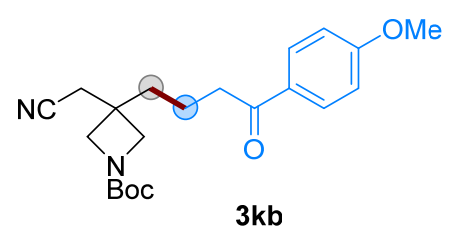

$49.7 \mathrm{mg}$, colorless oil, 67\% yield. ${ }^{1} \mathrm{H}$ NMR (400 $\left.\mathrm{MHz}, \mathrm{CDCl}_{3}\right) \delta(\mathrm{ppm}) 7.94(\mathrm{~d}, J=$ $8.97 \mathrm{~Hz}, 2 \mathrm{H}), 6.95(\mathrm{~d}, J=8.95 \mathrm{~Hz}, 2 \mathrm{H}), 3.88(\mathrm{~s}, 3 \mathrm{H}), 3.80-3.70$ (m, 4H), 3.00 (t, $J=$ 
$6.74 \mathrm{~Hz}, 2 \mathrm{H}), 2.69(\mathrm{~s}, 2 \mathrm{H}), 1.83-1.69(\mathrm{~m}, 4 \mathrm{H}), 1.44(\mathrm{~s}, 9 \mathrm{H}) .{ }^{13} \mathrm{C}$ NMR $\left(100 \mathrm{MHz}, \mathrm{CDCl}_{3}\right) \delta(\mathrm{ppm}) 197.7,163.6,156.2$, 130.3, 129.8, 117.1, 113.8, 80.0, 55.5, 37.6, 36.0, 35.6, 28.3, 28.2, 25.7, 18.6. IR (in KBr): 3130, 2968, 1696, 1602, 1400, $1169 \mathrm{~cm}^{-1}$. HRMS (EI): $\mathrm{m} / \mathrm{z}[\mathrm{M}+\mathrm{Na}]^{+}$calcd for $\mathrm{C}_{21} \mathrm{H}_{28} \mathrm{~N}_{2} \mathrm{NaO}_{4}: 395.1941$, found: 395.1938 .

\section{tert-butyl 4-(cyanomethyl)-4-(4-(4-methoxyphenyl)-4-oxobutyl)piperidine-1-carboxylate 3lb}<smiles>COc1ccc(C(=O)COCC2(CC#N)CCN(C(=O)OC(C)(C)C)CC2)cc1</smiles>

$52.1 \mathrm{mg}$, colorless oil, $65 \%$ yield. ${ }^{1} \mathrm{H}$ NMR $\left(400 \mathrm{MHz}, \mathrm{CDCl}_{3}\right) \delta(\mathrm{ppm}) 7.94(\mathrm{~d}, J=$ $8.80 \mathrm{~Hz}, 2 \mathrm{H}), 6.94(\mathrm{~d}, J=8.78 \mathrm{~Hz}, 2 \mathrm{H}), 3.88(\mathrm{~s}, 3 \mathrm{H}), 3.46-3.49(\mathrm{~m}, 3 \mathrm{H}), 2.98(\mathrm{t}, J=$ $6.72 \mathrm{~Hz}, 2 \mathrm{H}), 2.41(\mathrm{~s}, 2 \mathrm{H}), 1.74-1.45(\mathrm{~m}, 18 \mathrm{H}) .{ }^{13} \mathrm{C} \mathrm{NMR}\left(100 \mathrm{MHz}, \mathrm{CDCl}_{3}\right) \delta(\mathrm{ppm})$ 198.0, 163.5, 154.7, 130.2, 129.9, 117.5, 113.8, 79.7, 55.5, 38.0, 35.8, 34.3, 34.1, 28.4, 26.1, 17.5. IR (in KBr): 3420, 3130, 2361, 1681, 1400, $1169 \mathrm{~cm}^{-1}$. HRMS (EI): $\mathrm{m} / \mathrm{z}$ [M $+\mathrm{Na}]^{+}$calcd for $\mathrm{C}_{23} \mathrm{H}_{32} \mathrm{~N}_{2} \mathrm{NaO}_{4}: 423.2254$, found: 423.2249 .

\section{7-(4-methoxyphenyl)-2,2,4,4-tetramethyl-3,7-dioxoheptanenitrile 3mb}

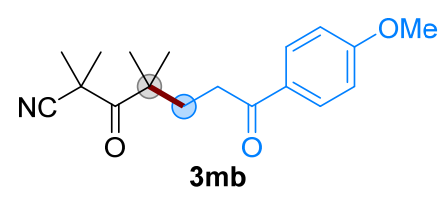

$38.2 \mathrm{mg}$, colorless oil, $63 \%$ yield. ${ }^{1} \mathrm{H}$ NMR $\left(400 \mathrm{MHz}, \mathrm{CDCl}_{3}\right) \delta(\mathrm{ppm}) 7.95(\mathrm{~d}, J=$ $8.92 \mathrm{~Hz}, 2 \mathrm{H}), 6.94(\mathrm{~d}, J=8.88 \mathrm{~Hz}, 2 \mathrm{H}), 3.87(\mathrm{~s}, 3 \mathrm{H}), 2.89(\mathrm{t}, 2 \mathrm{H}), 2.16(\mathrm{t}, 2 \mathrm{H}), 1.57$ (s, $6 \mathrm{H}), 1.47$ (s, 6H). ${ }^{13} \mathrm{C}$ NMR $\left(100 \mathrm{MHz}, \mathrm{CDCl}_{3}\right) \delta(\mathrm{ppm}) 208.1,197.8,163.4,130.3$, $129.7,123.2,113.7,55.5,49.3,40.1,34.1,33.7,26.7,24.4$. IR (in KBr): 3450,3131 , 2975, 1675, 1398, $1672 \mathrm{~cm}^{-1}$. HRMS (EI): $\mathrm{m} / \mathrm{z}[\mathrm{M}+\mathrm{Na}]^{+}$calcd for $\mathrm{C}_{18} \mathrm{H}_{23} \mathrm{NNaO}_{3}: 324.1570$, found: 324.1564 .

\section{2-(4-(4-methoxyphenyl)-4-oxobutoxy)acetonitrile 3nb}

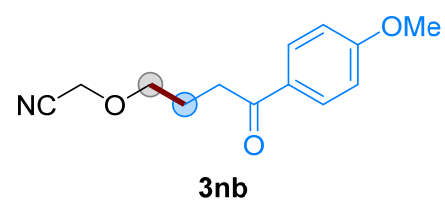

$37.3 \mathrm{mg}$, colorless oil, $81 \%$ yield. ${ }^{1} \mathrm{H}$ NMR (400 MHz, $\left.\mathrm{CDCl}_{3}\right) \delta(\mathrm{ppm}) 7.95(\mathrm{~d}, 2 \mathrm{H})$, $6.94(\mathrm{~d}, J=8.66 \mathrm{~Hz}, 2 \mathrm{H}), 4.25(\mathrm{~s}, 2 \mathrm{H}), 3.87(\mathrm{~s}, 3 \mathrm{H}), 3.72-3.63(\mathrm{~m}, 2 \mathrm{H}), 3.04(\mathrm{t}, J=$ $7.10 \mathrm{~Hz}, 2 \mathrm{H}), 2.10-2.04(\mathrm{~m}, 2 \mathrm{H}) .{ }^{13} \mathrm{C} \mathrm{NMR}\left(100 \mathrm{MHz}, \mathrm{CDCl}_{3}\right) \delta(\mathrm{ppm}) 197.8,163.4$, 130.2, 129.8, 116.0, 113.7, 71.0, 56.2, 55.4, 34.2, 23.7. IR (in KBr): 3454, 3130, 2941 , 1674, 1602, $1255 \mathrm{~cm}^{-1}$. HRMS (EI): $\mathrm{m} / \mathrm{z}[\mathrm{M}+\mathrm{Na}]^{+}$calcd for $\mathrm{C}_{13} \mathrm{H}_{15} \mathrm{NNaO}_{3}: 256.0944$, found: 256.0945 .

\section{2-(4-(4-methoxyphenyl)-4-oxobutyl)cyclopent-3-ene-1-carbonitrile 3ob}

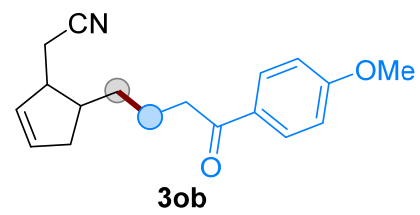

$39.2 \mathrm{mg}$, colorless oil, $73 \%$ yield. ${ }^{1} \mathrm{H}$ NMR $\left(400 \mathrm{MHz}, \mathrm{CDCl}_{3}\right) \delta(\mathrm{ppm}) 7.94(\mathrm{~d}, J=8.93$ $\mathrm{Hz}, 2 \mathrm{H}), 6.94(\mathrm{~d}, J=8.93 \mathrm{~Hz}, 2 \mathrm{H}), 5.86-5.83(\mathrm{~m}, 1 \mathrm{H}), 5.65-5.63(\mathrm{~m}, 1 \mathrm{H}), 3.87(\mathrm{~s}, 3 \mathrm{H})$, $2.97(\mathrm{t}, J=7.44 \mathrm{~Hz}, 2 \mathrm{H}), 2.71-2.65(\mathrm{~m}, 2 \mathrm{H}), 2.51-2.36(\mathrm{~m}, 2 \mathrm{H}), 2.11-1.94(\mathrm{~m}, 3 \mathrm{H})$, $1.81-1.73(\mathrm{~m}, 1 \mathrm{H}) .{ }^{13} \mathrm{C}$ NMR $\left(100 \mathrm{MHz}, \mathrm{CDCl}_{3}\right) \delta(\mathrm{ppm}) 198.4,163.4,132.5,130.7$, 130.3, 129.8, 118.8, 113.7, 55.5, 48.2, 43.1, 38.7, 36.3, 29.6, 22.8. IR (in KBr): 3417 , 3133, 2926,1671, 1257, $1173 \mathrm{~cm}^{-1}$. HRMS (EI): $\mathrm{m} / \mathrm{z}[\mathrm{M}+\mathrm{Na}]^{+}$calcd for $\mathrm{C}_{17} \mathrm{H}_{19} \mathrm{NNaO}_{2}: 292.1308$, found: 292.1307 .

\section{8-(4-methoxyphenyl)-5,5-dimethyl-8-oxooctanenitrile 3pb}

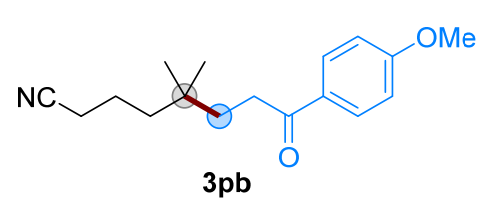

$34.7 \mathrm{mg}$, colorless oil, 63\% yield. ${ }^{1} \mathrm{H}$ NMR $\left(400 \mathrm{MHz}, \mathrm{CDCl}_{3}\right) \delta(\mathrm{ppm}) 7.94(\mathrm{~d}, J=$ $8.78 \mathrm{~Hz}, 2 \mathrm{H}), 6.94(\mathrm{~d}, 2 \mathrm{H}), 3.88(\mathrm{~s}, 3 \mathrm{H}), 2.87(\mathrm{t}, 2 \mathrm{H}), 2.34(\mathrm{t}, J=6.95 \mathrm{~Hz}, 2 \mathrm{H}), 1.69$ $-1.60(\mathrm{~m}, 4 \mathrm{H}), 1.42-1.38(\mathrm{~m}, 2 \mathrm{H}), 0.95(\mathrm{~s}, 6 \mathrm{H}) .{ }^{13} \mathrm{C} \mathrm{NMR}\left(100 \mathrm{MHz}, \mathrm{CDCl}_{3}\right) \delta$ (ppm) 199.2, 163.4, 130.3, 130.0, 119.8, 113.8, 55.5, 41.0, 35.9, 33.2, 32.6, 26.8, 20.4, 18.0. IR (in KBr): 3417, 3136, 2959, 1672, 1397, $1172 \mathrm{~cm}^{-1}$. HRMS (EI): m/z [M + Na] calcd for $\mathrm{C}_{17} \mathrm{H}_{23} \mathrm{NNaO}_{2}$ : 296.1621, found: 296.1620 .

\section{3-((5-(4-methoxyphenyl)-5-oxopentan-2-yl)oxy)propanenitrile 3qb}

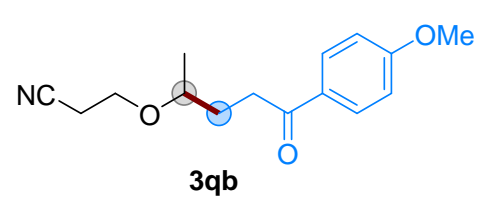

$29.8 \mathrm{mg}$, colorless oil, 57\% yield. ${ }^{1} \mathrm{H}$ NMR $\left(400 \mathrm{MHz}, \mathrm{CDCl}_{3}\right) \delta(\mathrm{ppm}) 7.97(\mathrm{~d}, J=$ $8.94 \mathrm{~Hz}, 2 \mathrm{H}), 6.93(\mathrm{~d}, J=8.93 \mathrm{~Hz}, 2 \mathrm{H}), 3.86(\mathrm{~s}, 3 \mathrm{H}), 3.81-3.71(\mathrm{~m}, 1 \mathrm{H}), 3.61-$ $3.53(\mathrm{~m}, 2 \mathrm{H}), 3.13-3.00(\mathrm{~m}, 2 \mathrm{H}), 2.56(\mathrm{t}, J=6.60 \mathrm{~Hz}, 2 \mathrm{H}), 1.98-1.86(\mathrm{~m}, 2 \mathrm{H})$, 
$1.21(\mathrm{~d}, J=6.13 \mathrm{~Hz}, 3 \mathrm{H}) .{ }^{13} \mathrm{C} \mathrm{NMR}\left(100 \mathrm{MHz}, \mathrm{CDCl}_{3}\right) \delta(\mathrm{ppm}) 198.8,163.4,130.4,130.0,118.2,113.7,75.5,63.0,55.5$, 33.8, 31.0, 19.6, 19.3. IR (in KBr): 3129, 2967, 1672, 1600, 1258, $1101 \mathrm{~cm}^{-1}$. HRMS (EI): $\mathrm{m} / \mathrm{z}[\mathrm{M}+\mathrm{Na}]^{+}$calcd for $\mathrm{C}_{15} \mathrm{H}_{19} \mathrm{NNaO}_{3}$ : 284.1257, found: 284.1257 .

\section{naphthalen-2-yl 6-cyano-5-(naphthalen-2-yl)hexanoate 3aa}

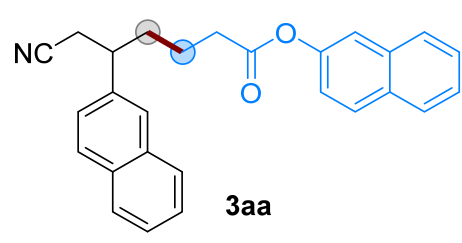

$52.7 \mathrm{mg}$, colorless oil, $67 \%$ yield. ${ }^{1} \mathrm{H}$ NMR $\left(400 \mathrm{MHz}, \mathrm{CDCl}_{3}\right) \delta$ (ppm) $7.83-7.76$ (m, 3H), $7.54-7.40(\mathrm{~m}, 3 \mathrm{H}), 7.20$ (d, $J=10.77 \mathrm{~Hz}, 1 \mathrm{H}), 2.53(\mathrm{t}, J=7.38 \mathrm{~Hz}, 2 \mathrm{H})$, $2.22(\mathrm{t}, J=6.97 \mathrm{~Hz}, 2 \mathrm{H}), 1.73-1.41(\mathrm{~m}, 6 \mathrm{H}) .{ }^{13} \mathrm{C} \mathrm{NMR}\left(100 \mathrm{MHz}, \mathrm{CDCl}_{3}\right) \delta(\mathrm{ppm})$ 171.6, 148.0, 133.4, 131.1, 129.1, 127.5, 127.3, 126.3, 125.4, 120.9, 119.4, 118.2, 33.6, 27.7, 24.7, 23.7, 16.6. IR (in KBr): 2939, 2245, 1720, 1755, $1131,750 \mathrm{~cm}^{-1}$. HRMS (EI): $\mathrm{m} / \mathrm{z}$ [M $+\mathrm{Na}]^{+}$calcd for $\mathrm{C}_{27} \mathrm{H}_{23} \mathrm{NNaO}_{2}$ : 416.1621, found: 416.1624 .

\section{methyl 6-cyano-2-methyl-5-(naphthalen-2-yl)hexanoate 3hc}

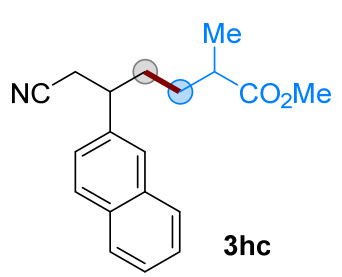

$59.0 \mathrm{mg}$, colorless oil, $64 \%$ yield (1:1 dr). Data of both isomers: ${ }^{1} \mathrm{H}$ NMR $\left(400 \mathrm{MHz}, \mathrm{CDCl}_{3}\right) \delta$ (ppm) $7.84(\mathrm{~m}, 8 \mathrm{H}), 7.66(\mathrm{~s}, 3 \mathrm{H}), 7.52-7.46(\mathrm{~m}, 5 \mathrm{H}), 7.33(\mathrm{~d}, J=8.51 \mathrm{~Hz}, 2 \mathrm{H}), 3.65(\mathrm{~s}, 8 \mathrm{H})$, $3.15-3.05(\mathrm{~m}, 2 \mathrm{H}), 2.70-2.67(\mathrm{~m}, 5 \mathrm{H}), 2.48-2.39(\mathrm{~m}, 2 \mathrm{H}), 1.88(\mathrm{dt}, J=12.32,5.67 \mathrm{~Hz}$, $5 \mathrm{H}), 1.54-1.26(\mathrm{~m}, 4 \mathrm{H}), 1.11-1.09(\mathrm{~m}, 8 \mathrm{H}) .{ }^{13} \mathrm{C} \mathrm{NMR}\left(100 \mathrm{MHz}, \mathrm{CDCl}_{3}\right) \delta(\mathrm{ppm}) 176.7$, 176.6, 138.6, 138.5, 133.5, 132.8, 128.9, 127.8, 127.7, 126.4, 126.4, 126.0, 124.6, 118.4, 51.7, $42.5,42.3,39.3,39.1,32.5,32.2,31.4,31.3,25.3,25.2,17.3$, 17.1. IR (in KBr): 3417,3133 , 1731, 1635, 1396, $1163 \mathrm{~cm}^{-1}$. HRMS (EI): $\mathrm{m} / \mathrm{z}[\mathrm{M}+\mathrm{Na}]^{+}$calcd for $\mathrm{C}_{19} \mathrm{H}_{21} \mathrm{NNaO}_{2}: 318.1465$, found: 318.1463 .

\section{phenyl 6-cyano-2-methyl-5-(naphthalen-2-yl)hexanoate 3hd}

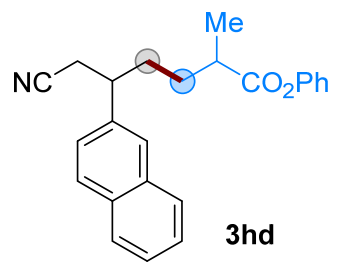

$52.1 \mathrm{mg}$, colorless oil, $73 \%$ yield (1:4 dr). ${ }^{1} \mathrm{H}$ NMR $\left(400 \mathrm{MHz}, \mathrm{CDCl}_{3}\right) \delta(\mathrm{ppm}) 7.87-7.69(\mathrm{~m}$, $4 \mathrm{H}$, major + minor $), 7.50-7.48(\mathrm{~m}, 2 \mathrm{H}$, major + minor $), 7.38-7.20(\mathrm{~m}, 4 \mathrm{H}$, major + minor $)$, 7.02 (dd, $J=8.23,2.09 \mathrm{~Hz}, 2 \mathrm{H}$, major + minor), $3.20-3.14$ (m, 1H, major), $3.13-3.10(\mathrm{~m}$, $1 \mathrm{H}$, minor), $2.76-2.69(\mathrm{~m}, 2 \mathrm{H}$, major), $2.67-2.54(\mathrm{~m}, 1 \mathrm{H}$, minor), $2.08-1.96(\mathrm{~m}, 2 \mathrm{H}$, major + minor), $1.82-1.39$ (m, 3H, major + minor), 1.26 (dd, $J=7.07,2.29 \mathrm{~Hz}, 3 \mathrm{H}$, major + minor). ${ }^{13} \mathrm{C} \mathrm{NMR}\left(100 \mathrm{MHz}, \mathrm{CDCl}_{3}\right) \delta$ (ppm, major + minor) $174.7,174.6,150.5,138.4,138.3,133.4,132.8,129.4,128.9,127.7$, 127.7, 126.4, 126.4, 126.0, 125.8, 124.6, 121.4, 118.4, 42.5, 42.3, 39.4, 39.4, 32.4, 32.2, 31.2, 31.2, 25.3, 25.2, 17.3, 17.0. IR (in KBr): 3130, 2936, 1752, 1393, 1193, $749 \mathrm{~cm}^{-1}$. HRMS (EI): $\mathrm{m} / \mathrm{z}[\mathrm{M}+\mathrm{Na}]^{+}$calcd for $\mathrm{C}_{24} \mathrm{H}_{23} \mathrm{NNaO}_{2}: 380.1621$, found: 380.1620 .

\section{naphthalen-2-yl 6-cyano-2-methyl-5-(naphthalen-2-yl)hexanoate 3he}

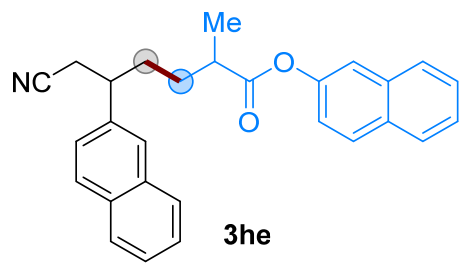

$58.9 \mathrm{mg}$, colorless oil, $72 \%$ yield (1:6 dr). Data of both isomers: ${ }^{1} \mathrm{H}$ NMR $(400 \mathrm{MHz}$, $\left.\mathrm{CDCl}_{3}\right) \delta(\mathrm{ppm}) 7.86-7.69(\mathrm{~m}, 9 \mathrm{H}$, major + minor $), 7.49-7.33(\mathrm{~m}, 7 \mathrm{H}$, major + minor), $7.15(\mathrm{~d}, 1 \mathrm{H}), 3.20-3.10(\mathrm{~m}, 1 \mathrm{H}$, major + minor), $2.79-2.76(\mathrm{~m}, 1 \mathrm{H}$, minor $)$, $2.73-2.68(\mathrm{~m}, 3 \mathrm{H}$, major), $2.11-1.94(\mathrm{~m}, 3 \mathrm{H}$, major + minor $), 1.85-1.39(\mathrm{~m}, 3 \mathrm{H}$, major + minor), $1.29\left(\mathrm{~d}, \mathrm{~J}=6.89 \mathrm{~Hz}, 4 \mathrm{H}\right.$, major + minor). ${ }^{13} \mathrm{C} \mathrm{NMR}(100 \mathrm{MHz}$, $\left.\mathrm{CDCl}_{3}\right) \delta(\mathrm{ppm}$, major + minor) $174.8,167.2,148.3,141.3,141.2,135.6,133.8,132.0$, $131.5,130.9,130.5,129.5,128.1,127.8,127.7,126.7,126.4,125.9,125.8,125.6,121.1,118.5,118.3,60.5,52.3,42.6$, 42.5, 39.5, 32.4, 32.3, 31.3, 25.2, 25.1, 21.1, 17.4, 17.1, 14.3. IR (in KBr): 3418, 3133, 1751, 1395, 1285, $1145 \mathrm{~cm}^{-1}$. HRMS (EI): $\mathrm{m} / \mathrm{z}[\mathrm{M}+\mathrm{Na}]^{+}$calcd for $\mathrm{C}_{28} \mathrm{H}_{25} \mathrm{NNaO}_{2}: 430.1778$, found: 430.1779 .

\section{3-(naphthalen-2-yl)-7-oxo-7-phenylheptanenitrile 3hf}

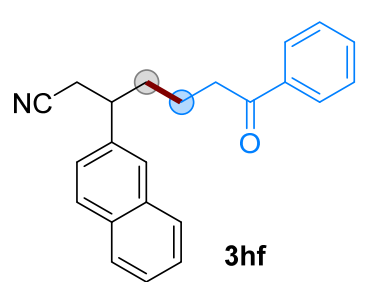

$46.1 \mathrm{mg}$, colorless oil, 71\% yield. ${ }^{1} \mathrm{H}$ NMR (400 MHz, $\left.\mathrm{CDCl}_{3}\right) \delta(\mathrm{ppm}) 7.89-7.81(\mathrm{~m}, 5 \mathrm{H})$, $7.70(\mathrm{~s}, 1 \mathrm{H}), 7.55-7.35(\mathrm{~m}, 7 \mathrm{H}), 3.21-3.13(\mathrm{~m}, 1 \mathrm{H}), 3.04-2.89(\mathrm{~m}, 2 \mathrm{H}), 2.71(\mathrm{~d}, J=6.92$ 
$\mathrm{Hz}, 2 \mathrm{H}), 2.05-1.92(\mathrm{~m}, 2 \mathrm{H}), 1.76-1.63(\mathrm{~m}, 2 \mathrm{H}) .{ }^{13} \mathrm{C} \mathrm{NMR}\left(100 \mathrm{MHz}, \mathrm{CDCl}_{3}\right) \delta(\mathrm{ppm})$ 199.5, 138.6, 136.6, 133.4, 133.0, 132.7, 128.8, 128.5, 127.9, 127.7, 127.6, 126.4, 126.3, 125.9, 124.7, 118.5, 42.3, 38.0, 34.3, 25.1, 21.8. IR (in $\mathrm{KBr}$ ): $3454,3130,2931,1681,1398,748 \mathrm{~cm}^{-1}$. HRMS (EI): $\mathrm{m} / \mathrm{z}[\mathrm{M}+\mathrm{Na}]^{+}$calcd for $\mathrm{C}_{23} \mathrm{H}_{21} \mathrm{NNaO}: 350.1515$, found: 350.1519 .

\section{3-(naphthalen-2-yl)-7-oxo-7-(p-tolyl)heptanenitrile 3hg}

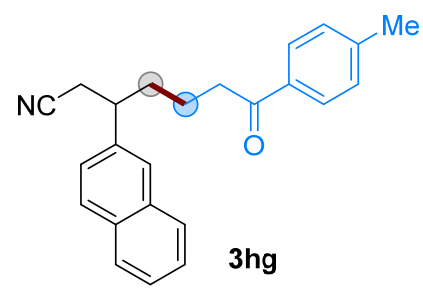

$42.8 \mathrm{mg}$, colorless oil, $63 \%$ yield. ${ }^{1} \mathrm{H}$ NMR $\left(400 \mathrm{MHz}, \mathrm{CDCl}_{3}\right) \delta(\mathrm{ppm}) 7.85-7.76(\mathrm{~m}$, 5H), $7.69(\mathrm{~s}, 1 \mathrm{H}), 7.50-7.44(\mathrm{~m}, 2 \mathrm{H}), 7.35(\mathrm{~d}, J=8.43 \mathrm{~Hz}, 1 \mathrm{H}), 7.20(\mathrm{~d}, J=7.91 \mathrm{~Hz}$, 2H), $3.19-3.12(\mathrm{~m}, 1 \mathrm{H}), 3.00-2.87(\mathrm{~m}, 2 \mathrm{H}), 2.70(\mathrm{~d}, J=6.90 \mathrm{~Hz}, 2 \mathrm{H}), 2.38(\mathrm{~s}, 3 \mathrm{H})$, $2.00-1.93(\mathrm{~m}, 2 \mathrm{H}), 1.76-1.64(\mathrm{~m}, 2 \mathrm{H}) .{ }^{13} \mathrm{C} \mathrm{NMR}\left(100 \mathrm{MHz}, \mathrm{CDCl}_{3}\right) \delta(\mathrm{ppm}) 199.3$, $143.9,138.7,134.3,133.5,132.8,129.3,128.9,128.1,127.8,127.7,126.4,126.4,126.0$, $124.8,118.6,42.4,38.0,34.4,25.2$, 22.0, 21.7. IR (in KBr): 3421, 3130, 2931, 1678, 1399, $815 \mathrm{~cm}^{-1}$. HRMS (EI): $\mathrm{m} / \mathrm{z}[\mathrm{M}+\mathrm{Na}]^{+}$calcd for $\mathrm{C}_{24} \mathrm{H}_{23} \mathrm{NNaO}$ : 364.1672, found: 364.1666 .

\section{3-(naphthalen-2-yl)-7-oxo-6,7-diphenylheptanenitrile 3hh}

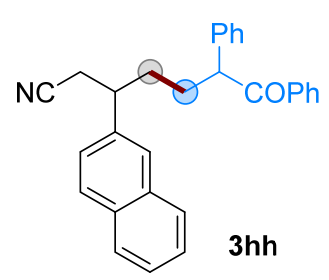

$63.9 \mathrm{mg}$, colorless oil, $79 \%$ yield $(1: 1.3 \mathrm{dr}) .{ }^{1} \mathrm{H}$ NMR $\left(400 \mathrm{MHz}, \mathrm{CDCl}_{3}\right) \delta(\mathrm{ppm}) 7.88-7.85(\mathrm{~m}$, $5 \mathrm{H}$, major + minor $), 7.83-7.78(\mathrm{~m}, 4 \mathrm{H}$, major + minor $), 7.76-7.74(\mathrm{~m}, 2 \mathrm{H}$, major + minor $)$, $7.65-7.57(\mathrm{~d}, 2 \mathrm{H}$, major + minor), $7.49-7.41(\mathrm{~m}, 7 \mathrm{H}$, major + minor), $7.34-7.15(\mathrm{~m}, 19 \mathrm{H}$, major + minor), $4.50-4.48(\mathrm{~m}, 1 \mathrm{H}$, minor $), 4.47-4.45(\mathrm{~m}, 1 \mathrm{H}$, major $), 3.14-3.03(\mathrm{~m}, 2 \mathrm{H}$, major + minor $), 2.61-2.58(\mathrm{~m}, 4 \mathrm{H}$, major + minor $), 2.18-1.69(\mathrm{~m}, 9 \mathrm{H}$, major + minor $) .{ }^{13} \mathrm{C}$ NMR $\left(100 \mathrm{MHz}, \mathrm{CDCl}_{3}\right) \delta$ (ppm, major + minor) 199.4, 199.3, 139.2, 138.9, 138.6, 138.6, 136.5, 136.5, 133.5, 133.5, 133.0, 133.0, 132.8, 132.8, 129.1, 129.0, 128.9, 128.9, 128.7, 128.7, 128.6, 128.2, 128.1, $127.9,127.8,127.7,127.7,127.3,126.5,126.4,126.0,124.8,124.8,118.5,53.6,53.3,42.6,42.5,32.8,32.6,31.8,31.6$, 25.4, 25.2. IR (in KBr): $3058,2929,1678,1396,749,698 \mathrm{~cm}^{-1}$. HRMS (EI): $\mathrm{m} / \mathrm{z}[\mathrm{M}+\mathrm{Na}]^{+}$calcd for $\mathrm{C}_{29} \mathrm{H}_{25} \mathrm{NNaO}$ : 426.1828, found: 426.1827 .

\section{3-(naphthalen-2-yl)-7-oxononanenitrile 3hi}

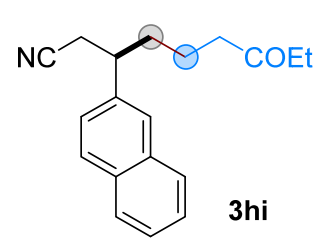

$33.2 \mathrm{mg}$, colorless oil, $59 \%$ yield. ${ }^{1} \mathrm{H}$ NMR $\left(400 \mathrm{MHz}, \mathrm{CDCl}_{3}\right) \delta(\mathrm{ppm}) 7.85-7.80(\mathrm{~m}, 3 \mathrm{H})$, $7.67(\mathrm{~s}, 1 \mathrm{H}), 7.51-7.45(\mathrm{~m}, 2 \mathrm{H}), 7.33(\mathrm{dd}, J=8.48,1.74 \mathrm{~Hz}, 1 \mathrm{H}), 3.15-3.08(\mathrm{~m}, 1 \mathrm{H}), 2.69$ (d, $2 \mathrm{H}), 2.42-2.33(\mathrm{~m}, 4 \mathrm{H}), 1.91-1.83(\mathrm{~m}, 2 \mathrm{H}), 1.58-1.44(\mathrm{~m}, 2 \mathrm{H}), 1.01(\mathrm{t}, J=7.33 \mathrm{~Hz}, 3 \mathrm{H}) .{ }^{13} \mathrm{C}$ NMR $\left(100 \mathrm{MHz}, \mathrm{CDCl}_{3}\right) \delta(\mathrm{ppm}) 211.0,138.7,133.5,132.8,128.9,127.8,127.7,126.4,126.3$, 126.0, 124.7, 118.5, 42.4, 41.8, 35.9, 34.3, 25.1, 21.5, 7.8. IR (in KBr): 3130, 1709, 1397, 1112 , $820,753 \mathrm{~cm}^{-1}$. HRMS (EI): $\mathrm{m} / \mathrm{z}[\mathrm{M}+\mathrm{Na}]^{+}$calcd for $\mathrm{C}_{19} \mathrm{H}_{21} \mathrm{NNaO}: 320.1515$, found: 320.1516 .

\section{6-cyano-5-(naphthalen-2-yl)-N-phenyl-2-(p-tolyl)hexanamide 3hj}

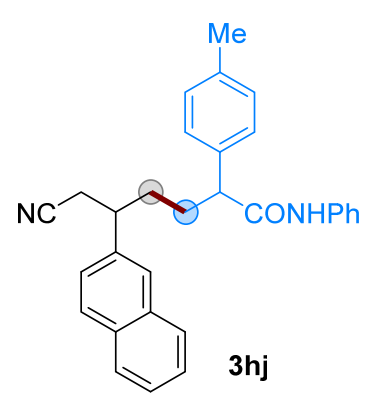

$37.4 \mathrm{mg}$, colorless oil, $61 \%$ yield (1:1.3 dr). Data of both isomers: ${ }^{1} \mathrm{H}$ NMR $(400 \mathrm{MHz}$, $\left.\mathrm{CDCl}_{3}\right) \delta(\mathrm{ppm}) 7.83-7.75(\mathrm{~m}, 3 \mathrm{H}), 7.61(\mathrm{~d}, J=20.16 \mathrm{~Hz}, 1 \mathrm{H}), 7.48-7.33(\mathrm{~m}, 5 \mathrm{H}), 7.23-$ $7.02(\mathrm{~m}, 7 \mathrm{H}), 3.40(\mathrm{t}, J=7.10 \mathrm{~Hz}, 1 \mathrm{H}), 3.12-3.05(\mathrm{~m}, 1 \mathrm{H}), 2.62(\mathrm{t}, 2 \mathrm{H}), 2.32(\mathrm{~s}, 3 \mathrm{H}), 2.24-$ $1.72(\mathrm{~m}, 4 \mathrm{H}) .{ }^{13} \mathrm{C}$ NMR $\left(100 \mathrm{MHz}, \mathrm{CDCl}_{3}\right) \delta(\mathrm{ppm}) 171.3,171.3,138.6,138.5,137.7,137.7$, $137.6,137.5,136.0,135.7,133.5,132.8,129.9,129.9,128.9,128.9,128.9,127.9,127.9$, $127.8,127.8,127.8,127.7,126.4,126.4,126.4,126.0,126.0,124.8,124.8,124.3,119.7$, $119.7,118.5,118.5,53.6,53.6,42.5,42.5,32.9,32.8,30.9,30.7,25.4,25.0,21.1$. IR (in $\mathrm{KBr}): 3100,1678,1531,1398,1290,748 \mathrm{~cm}^{-1}$. HRMS (EI): $\mathrm{m} / \mathrm{z}[\mathrm{M}+\mathrm{Na}]^{+}$calcd for $\mathrm{C}_{30} \mathrm{H}_{28} \mathrm{~N}_{2} \mathrm{NaO}: 455.2094$, found: 455.2092 .

\section{methyl 6-cyano-2-methylene-5-(naphthalen-2-yl)hexanoate 3hk}




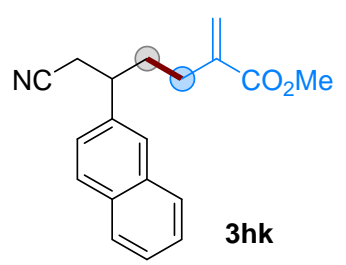

$37.6 \mathrm{mg}$, colorless oil, 64\% yield. ${ }^{1} \mathrm{H}$ NMR $\left(400 \mathrm{MHz}, \mathrm{CDCl}_{3}\right) \delta(\mathrm{ppm}) 7.87-7.82(\mathrm{~m}, 3 \mathrm{H})$, $7.69(\mathrm{~s}, 1 \mathrm{H}), 7.51-7.46(\mathrm{~m}, 2 \mathrm{H}), 7.35$ (d, J = 8.41 Hz, 1H), $6.15(\mathrm{~s}, 1 \mathrm{H}), 5.49$ (s, 1H), 3.72 (s, $3 \mathrm{H}), 3.18-3.11(\mathrm{~m}, 1 \mathrm{H}), 2.70(\mathrm{~d}, J=6.89 \mathrm{~Hz}, 2 \mathrm{H}), 2.11-2.05(\mathrm{~m}, 4 \mathrm{H}) .{ }^{13} \mathrm{C} \mathrm{NMR}(100 \mathrm{MHz}$, $\left.\mathrm{CDCl}_{3}\right) \delta(\mathrm{ppm}) 167.3,139.5,138.4,133.5,132.9,129.0,127.8,127.7,126.5,126.4,126.1$, 125.7, 124.8, 118.4, 51.9, 41.9, 33.4, 29.9, 25.3. IR (in KBr): 3130, 1717, 1633, 1397, 1197 , $820 \mathrm{~cm}^{-1}$. HRMS (EI): $\mathrm{m} / \mathrm{z}[\mathrm{M}+\mathrm{Na}]^{+}$calcd for $\mathrm{C}_{19} \mathrm{H}_{19} \mathrm{NNaO}_{2}: 316.1308$, found: 316.1309 .

\section{Preparative Utility of the Methodology}

\subsection{0 mmol Reaction}

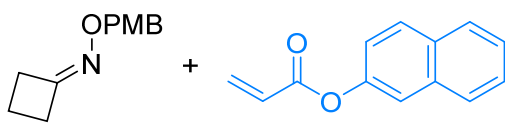

1a-I, $1.0 \mathrm{mmo}$

2a, $2.0 \mathrm{mmol}$

\section{2-Chloroanthraquinone ( 0.50 equiv) \\ $\mathrm{K}_{2} \mathrm{CO}_{3}$ (2.0 equiv),}

2-butanone (20.0 mL)

$370 \mathrm{~nm}$ LED, $25^{\circ} \mathrm{C}, 12 \mathrm{~h}$

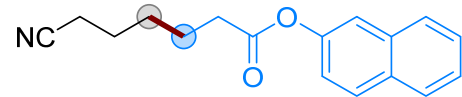

3aa

In a dried sealed tube, 1a-I (205.30mg, $1.0 \mathrm{mmol}), 2 \mathbf{a}(396.14 \mathrm{mg}, 2.0 \mathrm{mmol}), 2$-chloroanthraquinone (0.50 equiv) and $\mathrm{K}_{2} \mathrm{CO}_{3}$ (2.0 equiv) were dissolved in 2-butanone $(20.0 \mathrm{~mL})$ under a $\mathrm{Ar}$ atmosphere. Then, the resulting mixture was degassed via 'freeze-pump-thaw' procedure ( 3 times). After that, the solution was stirred at a distance of $\sim 5 \mathrm{~cm}$ from a $370 \mathrm{~nm}$ LED at room temperature about $12 \mathrm{~h}$ until the reaction was completed as monitored by TLC analysis. The crude product was purified by flash chromatography on silica gel (petroleum ether/ethylacetate 5:1) directly to give the desired product 3aa in $69 \%$ yield (184.46 mg) as a colorless oil.

\subsection{Gram-scale reaction}

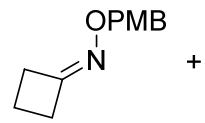

1a-I, $5.0 \mathrm{mmol}$

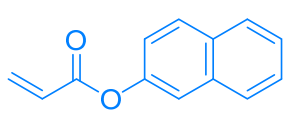

2a, $10.0 \mathrm{mmol}$

\section{2-Chloroanthraquinone ( 0.50 equiv) \\ $\mathrm{K}_{2} \mathrm{CO}_{3}$ (2.0 equiv),}

2-butanone (100.0 mL)

$370 \mathrm{~nm}$ LED, $25^{\circ} \mathrm{C}, 12 \mathrm{~h}$

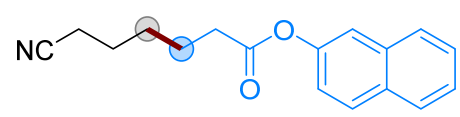

3aa

In a dried sealed tube, 1a-I (1.03 g, $5.0 \mathrm{mmol}), 2 \mathbf{b}(1.98 \mathrm{~g}, 10.0 \mathrm{mmol})$, 2-Chloroanthraquinone (0.50 equiv) and $\mathrm{K}_{2} \mathrm{CO}_{3}$ (2.0 equiv) were dissolved in 2-butanone $(100.0 \mathrm{~mL})$ under a Ar atmosphere. Then, the resulting mixture was degassed via 'freeze-pump-thaw' procedure (3 times). After that, the solution was stirred at a distance of $\sim 5 \mathrm{~cm}$ from a $370 \mathrm{~nm}$ LED at room temperature about $12 \mathrm{~h}$ until the reaction was completed as monitored by TLC analysis. The crude product was purified by flash chromatography on silica gel (petroleum ether/ethylacetate 5:1) directly to give the desired product 3aa in $64 \%$ yield $(0.85 \mathrm{~g})$ as a colorless oil. ${ }^{1} \mathrm{H}$ NMR $\left(400 \mathrm{MHz}, \mathrm{CDCl}_{3}\right) \delta(\mathrm{ppm}) 7.86-7.82(\mathrm{~m}, 4 \mathrm{H}), 7.51-7.44(\mathrm{~m}$, $3 \mathrm{H}), 2.35(\mathrm{dt}, J=41.98,7.22 \mathrm{~Hz}, 4 \mathrm{H}), 1.73-1.62(\mathrm{~m}, 4 \mathrm{H}), 1.51-1.44(\mathrm{~m}, 2 \mathrm{H}) .{ }^{13} \mathrm{C} \mathrm{NMR}\left(100 \mathrm{MHz}, \mathrm{CDCl}_{3}\right) \delta(\mathrm{ppm})$ 174.1, 149.4, 134.7, 132.4, 129.4, 127.7, 127.6, 126.4, 126.1, 119.8, 118.9, 117.7, 34.4, 28.1, 25.8, 25.4, 18.4. HRMS (EI): $\mathrm{m} / \mathrm{z}[\mathrm{M}+\mathrm{Na}]^{+}$calcd for $\mathrm{C}_{17} \mathrm{H}_{17} \mathrm{NNaO}_{2}: 290.1151$, found: 290.1151 .

\subsection{Alkynylation of cyanoalkyl radicals}<smiles>COc1cc(CON=C2CCC2)cc(OC)c1OC</smiles>

1a-III, $0.2 \mathrm{mmol}$

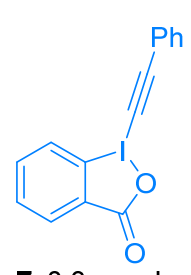

$7,0.3 \mathrm{mmol}$

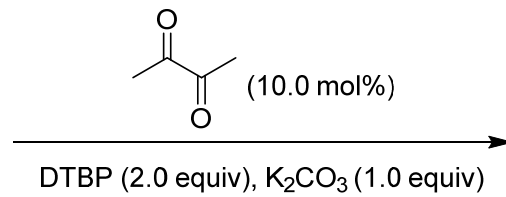

DCE (0.05 M), $390 \mathrm{~nm}$ Light , $16 \mathrm{~h}$

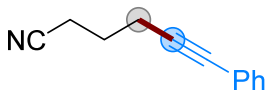


In a dried sealed tube, 1a-III (53.06 mg, $0.2 \mathrm{mmol}), 7$ (104.44 mg, $0.3 \mathrm{mmol}$ ), DTBP (2.0 equiv), 2,3-Butanedione (10.0 mol\%) and $\mathrm{K}_{2} \mathrm{CO}_{3}$ (1.0 equiv) were dissolved in DCE $(4.0 \mathrm{~mL})$. Then, the resulting mixture was degassed via 'freeze-pump-thaw' procedure ( 3 times). After that, the solution was stirred at a distance of $\sim 5 \mathrm{~cm}$ from a $390 \mathrm{~nm}$ LED at room temperature about $16 \mathrm{~h}$ until the reaction was completed as monitored by TLC analysis. The crude product was purified by flash chromatography on silica gel (petroleum ether/ethylacetate 10:1) directly to give the desired product 8 in $68 \%$ yield $(23.01 \mathrm{mg})$ as a colorless oil. ${ }^{1} \mathrm{H}$ NMR $\left(400 \mathrm{MHz}, \mathrm{CDCl}_{3}\right) \delta(\mathrm{ppm}) 7.41-7.39(\mathrm{~m}, 2 \mathrm{H}), 7.31-7.29(\mathrm{~m}, 3 \mathrm{H})$, $2.63-2.56(\mathrm{~m}, 4 \mathrm{H}), 2.01-1.94(\mathrm{~m}, 2 \mathrm{H}) .{ }^{13} \mathrm{C} \mathrm{NMR}\left(100 \mathrm{MHz}, \mathrm{CDCl}_{3}\right) \delta(\mathrm{ppm}) 132.1,128.9,128.6,123.7,119.8,87.5$, 83.0, 25.2, 19.1, 16.8. HRMS (EI): $\mathrm{m} / \mathrm{z}[\mathrm{M}+\mathrm{Na}]^{+}$calcd for $\mathrm{C}_{12} \mathrm{H}_{11} \mathrm{NNa}$ : 192.0784, found: 192.0784 .

\section{The Mechanism Studies and Spectral Data of Products}

\subsection{TEMPO Trapping Experiment}

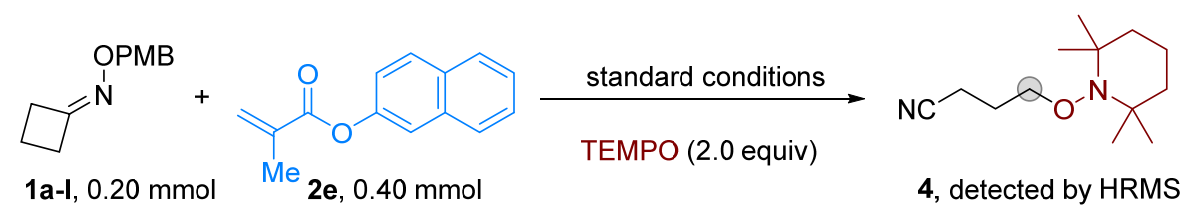

In a dried sealed tube, 1a-I $(41.06 \mathrm{mg}, 0.20 \mathrm{mmol}), \mathbf{2 e}(84.90 \mathrm{mg}, 0.40 \mathrm{mmol}), 2$-chloroanthraquinone (24.27 $\mathrm{mg}, 0.50$ equiv) and $\mathrm{K}_{2} \mathrm{CO}_{3}(55.28 \mathrm{mg}, 2.0$ equiv) were dissolved in 2-butanone $(4.0 \mathrm{~mL})$ under a Ar atmosphere. Then TEMPO (2.0 equiv) was added into system. Then, the resulting mixture was degassed via 'freeze-pump-thaw' procedure ( 3 times). After that, the solution was stirred at a distance of $\sim 5 \mathrm{~cm}$ from a $370 \mathrm{~nm}$ LED at room temperature about $12 \mathrm{~h}$ until the reaction was completed as monitored by TLC analysis. The crude product was purified by flash chromatography on silica gel (petroleum ether/ethylacetate 5:1) directly to give the desired product 3ae with trace yield. The corresponding trapping product 4 was detected by HRMS. HRMS (EI): $\mathrm{m} / \mathrm{z}[\mathrm{M}+\mathrm{H}]^{+}$calcd for $\mathrm{C}_{13} \mathrm{H}_{25} \mathrm{~N}_{2} \mathrm{O}: 225.1961$, found: 225.1960 .

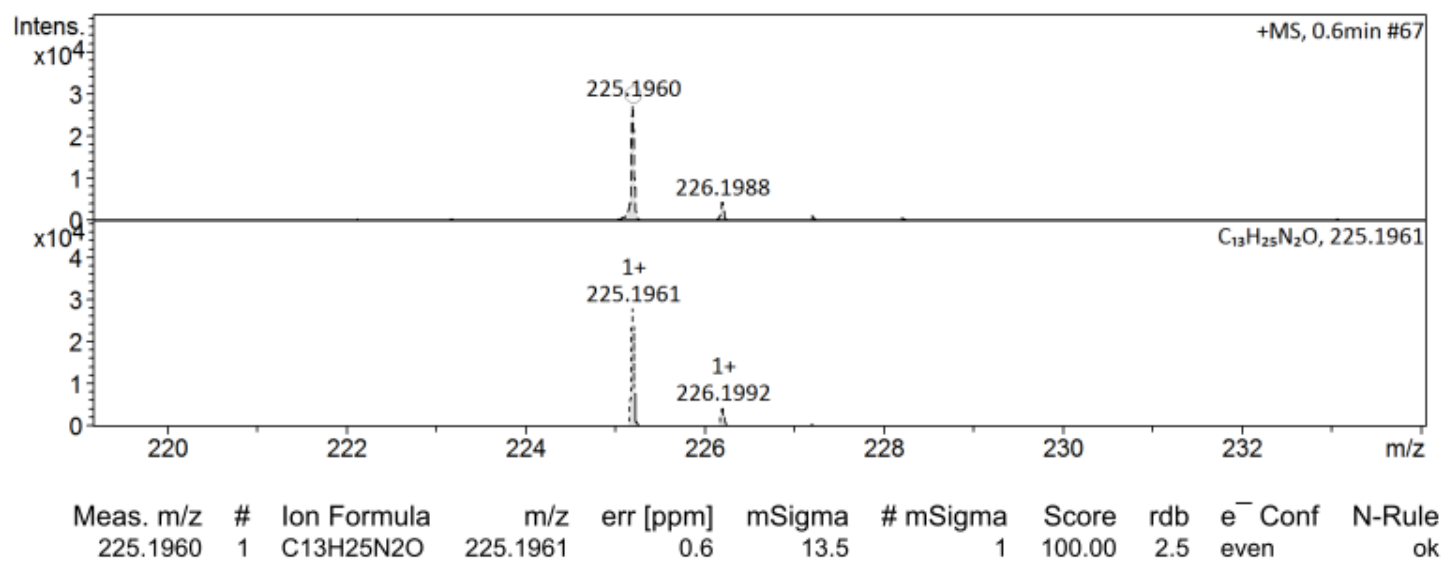

\subsection{PhSeSePh Trapping Experiment}

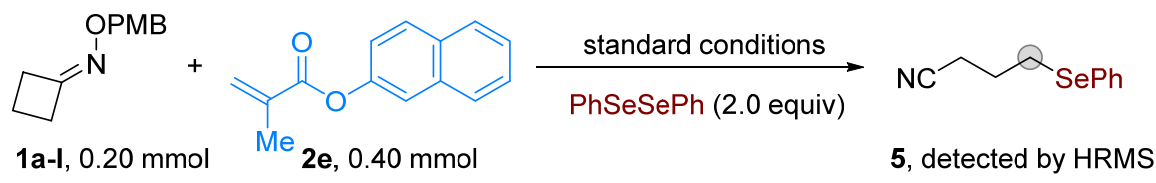

In a dried sealed tube, 1a-I $(41.06 \mathrm{mg}, 0.20 \mathrm{mmol}), \mathbf{2 e}(84.90 \mathrm{mg}, 0.40 \mathrm{mmol}), 2$-chloroanthraquinone (24.27 $\mathrm{mg}, 0.50$ equiv) and $\mathrm{K}_{2} \mathrm{CO}_{3}(55.28 \mathrm{mg}, 2.0$ equiv) were dissolved in 2-butanone $(4.0 \mathrm{~mL})$ under a Ar atmosphere. Then TEMPO (2.0 equiv) was added into system. Then, the resulting mixture was degassed via 'freeze-pump-thaw' procedure ( 3 times). 
After that, the solution was stirred at a distance of $\sim 5 \mathrm{~cm}$ from a $370 \mathrm{~nm}$ LED at room temperature about $12 \mathrm{~h}$ until the reaction was completed as monitored by TLC analysis. The crude product was purified by flash chromatography on silica gel (petroleum ether/ethylacetate 5:1) directly to give the desired product 3ae with trace yield. The corresponding trapping product 5 was detected by HRMS. HRMS (EI): $\mathrm{m} / \mathrm{z}[\mathrm{M}+\mathrm{Na}]^{+}$calcd for $\mathrm{C}_{10} \mathrm{H}_{11} \mathrm{NNaSe}$ : 247.9949, found: 247.9950.

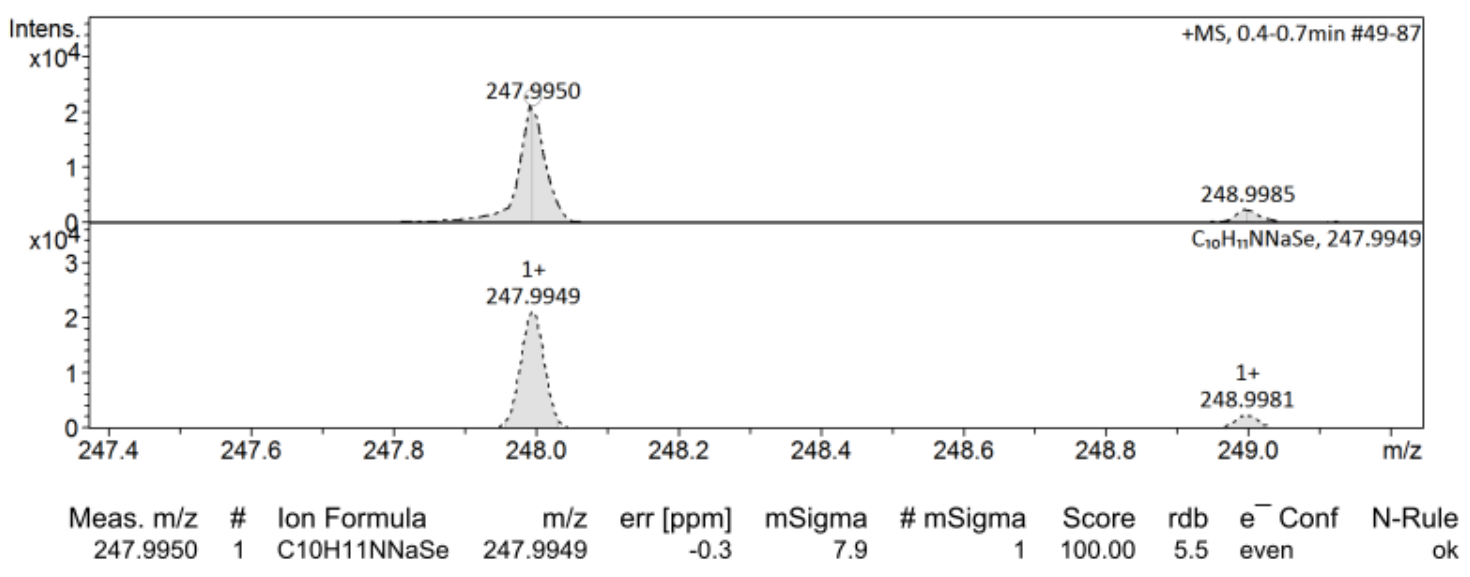

\subsection{Isotope Labeling Experiment}

The $\left[\mathbf{D}_{2}\right]-\mathbf{1 a}-\mathbf{I}$ was synthesized from benzenemethan- $d_{2}$-ol according to the literature. ${ }^{[3]}$

Deuterium incorporation was detected to be over $99 \%$ by ${ }^{1} \mathrm{H} \mathrm{NMR}$ in $\mathrm{CDCl}_{3}$.

${ }^{1} \mathrm{H}$ NMR (400 MHz, CDCl3) spectra of [D $]$-1a-I

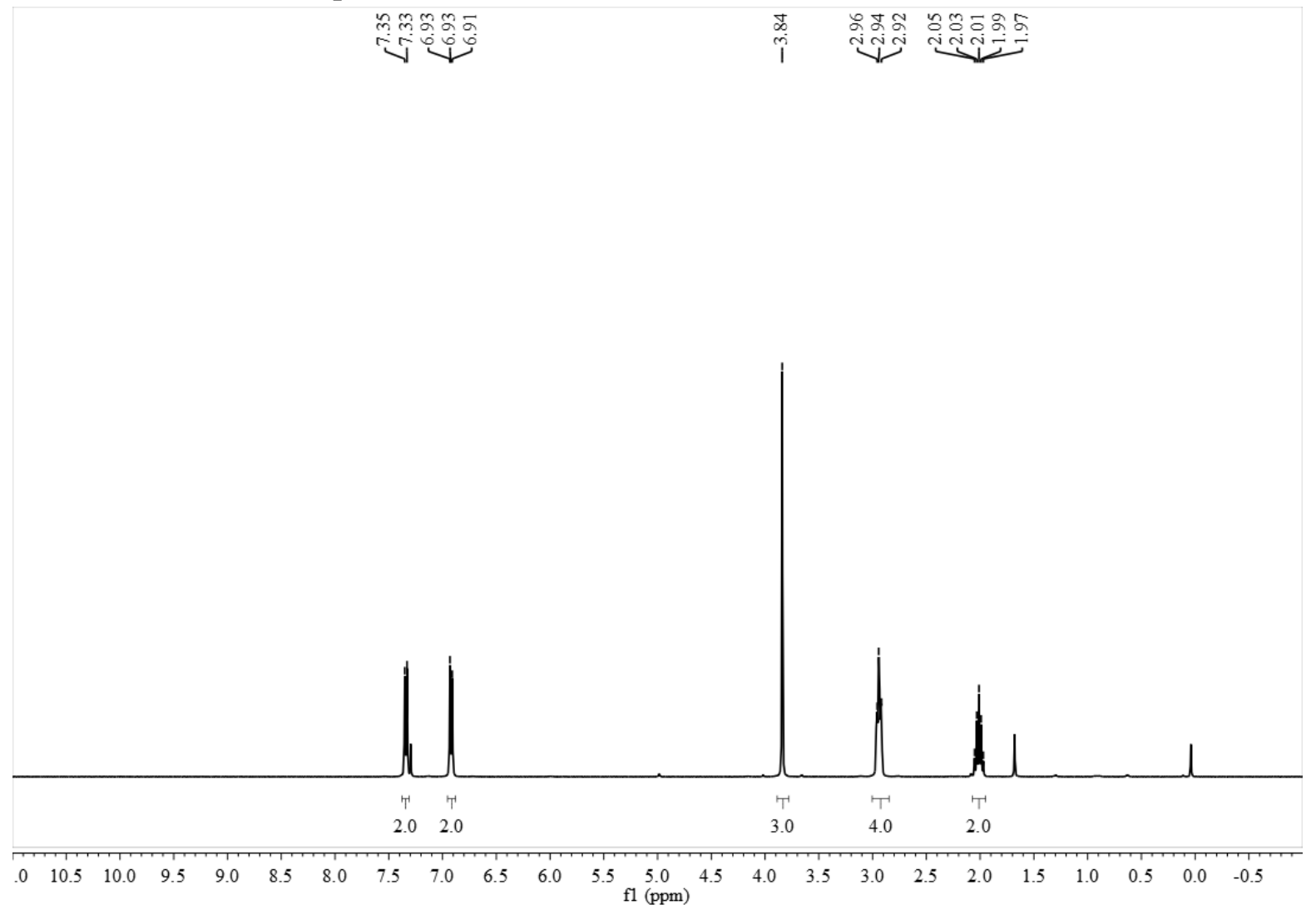




\section{Competitive Experiment}

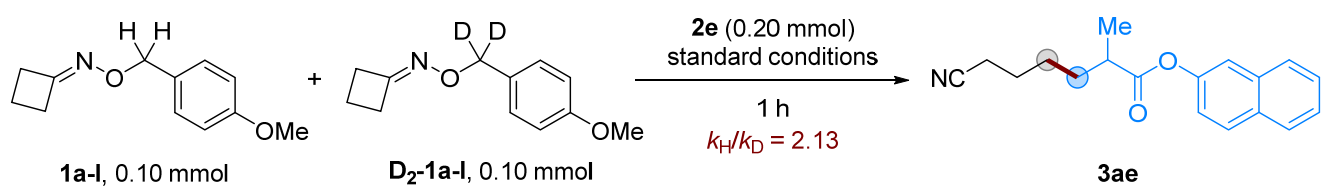

\section{Parallel Experiment}

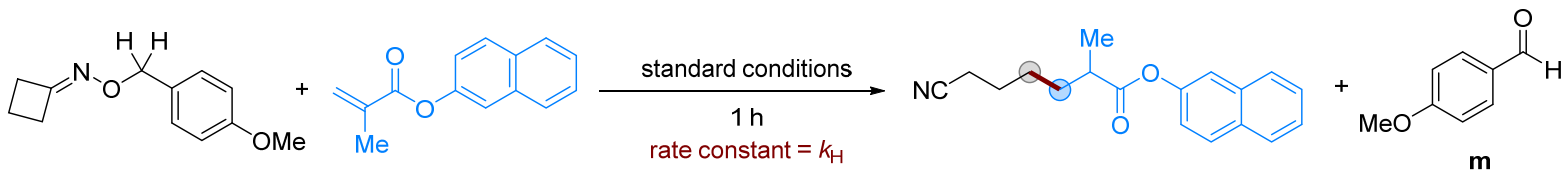

1a-I, $0.20 \mathrm{mmol}$ $2 e, 0.40 \mathrm{mmol}$<smiles>[2H]C([2H])(ON=C1CCC1)c1ccc(OC)cc1</smiles>

$\mathrm{D}_{\mathbf{2}}-1 \mathrm{a}-\mathrm{I}, 0.20 \mathrm{mmol}$

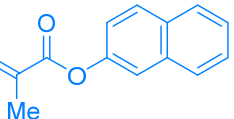

$2 \mathrm{e}, 0.40 \mathrm{mmol}$ rate constant $=k_{H}$<smiles>CC(OCCC#N)C(=O)Oc1ccc2ccccc2c1</smiles>

3ae

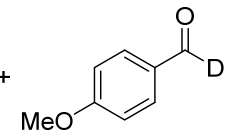

$\mathrm{m}_{\mathbf{D}}$

\section{Intermolecular Competitive Kinetic Isotope Effect (KIE) Experiment}

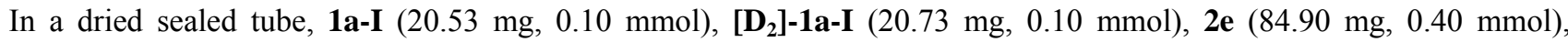
2-chloroanthraquinone (24.27 mg, 0.50 equiv) and $\mathrm{K}_{2} \mathrm{CO}_{3}(55.28 \mathrm{mg}, 2.0$ equiv) were dissolved in 2-butanone (4.0 mL) under a Ar atmosphere. Then, the resulting mixture was degassed via 'freeze-pump-thaw' procedure ( 3 times). After that, the solution was stirred at a distance of $\sim 5 \mathrm{~cm}$ from a $370 \mathrm{~nm}$ LED at room temperature about $1 \mathrm{~h}$. After that, the solvent was concentrated in vacuo and residual starting material was characterized by ${ }^{1} \mathrm{H}$ NMR spectra. Peak areas according to ${ }^{1} \mathrm{H}-\mathrm{NMR}$ were compared to give the ratio $(0.72: 0.28)$ of $\left[\mathbf{D}_{\mathbf{2}}\right] \mathbf{- 1} \mathbf{- 1}-\mathbf{I}$ to $\mathbf{1 a - I}$ in residual material. $k_{\mathrm{H}} / k_{\mathrm{D}}$ was calculated using the following expression ${ }^{[4]}: k_{\mathrm{H}} / k_{\mathrm{D}}=(\mathrm{M} / 2-0.48 \mathrm{~m}) /(\mathrm{M} / 2-0.52 \mathrm{~m})$

$\mathrm{M}, \mathrm{m}$ represent the amount of deuterated oxime ether $\left[\mathbf{D}_{2}\right]-\mathbf{1} \mathbf{a}-\mathbf{I}$ and oxime ether $\mathbf{1 a}-\mathbf{I}$ in reaction staring material and residual material, respectively. Here, $\mathrm{M}=41.26, \mathrm{~m}=18.61$, which corresponds to $k_{\mathrm{H}} / k_{\mathrm{D}}=2.13$.

\section{Intermolecular Parallel Kinetic Isotope Effect (KIE) experiment:}

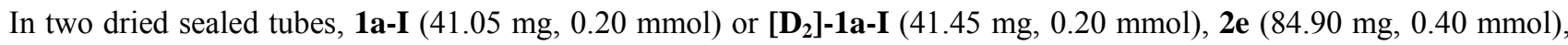
2-chloroanthraquinone (24.27 mg, 0.50 equiv) and $\mathrm{K}_{2} \mathrm{CO}_{3}$ (55.28 mg, 2.0 equiv) were dissolved in 2-butanone (4.0 mL) under a Ar atmosphere. Then, the resulting mixture was degassed via 'freeze-pump-thaw' procedure ( 3 times). After that, the solution was stirred at a distance of $\sim 5 \mathrm{~cm}$ from a $370 \mathrm{~nm}$ LED at room temperature about $1 \mathrm{~h}$. After that, the solvent were concentrated in vacuo and the residue were purified by chromatography column on silica gel to give the corresponding products 3ae (24.40 mg for 1a-I and $11.19 \mathrm{mg}$ for $\left.\left[\mathbf{D}_{2}\right]-\mathbf{1 a}-\mathbf{I}\right) . k_{\mathrm{H}} / k_{\mathrm{D}}$ was calculated using the following expression: $k_{\mathrm{H}} / k_{\mathrm{D}}=\mathrm{m} / \mathrm{m}_{\mathrm{D}}$

$\mathrm{m}, \mathrm{m}_{\mathrm{D}}$ represent the amount of corresponding aldehyde with $\mathbf{1 a - I}$ or $\left[\mathbf{D}_{\mathbf{2}}\right] \mathbf{- 1} \mathbf{- 1}-\mathbf{I}$ as starting material, respectively. Here, $\mathrm{m}$ $=24.40 \mathrm{mg}, \mathrm{m}_{\mathrm{D}}=11.19 \mathrm{mg}$, which corresponds to $k_{\mathrm{H}} / k_{\mathrm{D}}=2.18$.

\subsection{Deuterium Labeling Experiments}

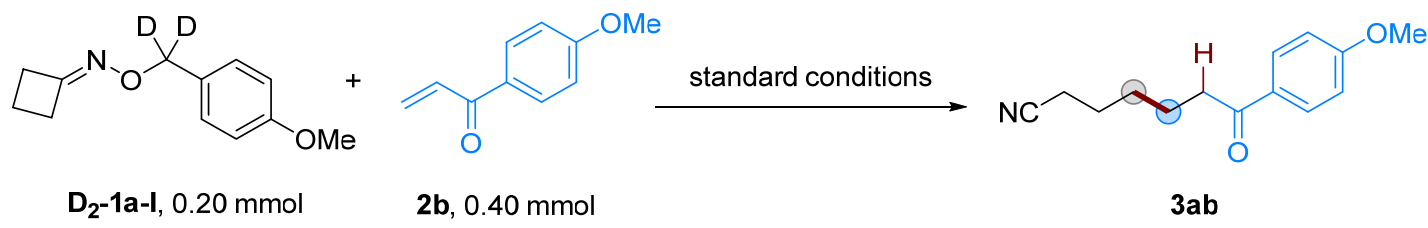

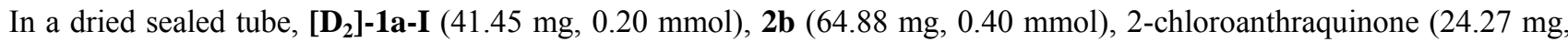
0.50 equiv) and $\mathrm{K}_{2} \mathrm{CO}_{3}$ (55.28 mg, 2.0 equiv) were dissolved in 2-butanone (4.0 mL) under a Ar atmosphere. Then, the 
resulting mixture was degassed via 'freeze-pump-thaw' procedure (3 times). After that, the solution was stirred at a distance of $\sim 5 \mathrm{~cm}$ from a $370 \mathrm{~nm}$ LED at room temperature about $12 \mathrm{~h}$ until the reaction was completed as monitored by TLC analysis. The crude product was purified by flash chromatography on silica gel (petroleum ether/ethylacetate 5:1) directly to give the desired product 3ab with $72 \%$ yield as a colorless oil.

No deuterated product 3ab was detected by ${ }^{1} \mathrm{H}-\mathrm{NMR}$, which suggests the hydrogen of product 3ab does not come from the benzylic hydrogen of the oxime ethers $\left[\mathbf{D}_{2}\right]-\mathbf{1} \mathbf{a}-\mathbf{I}$.

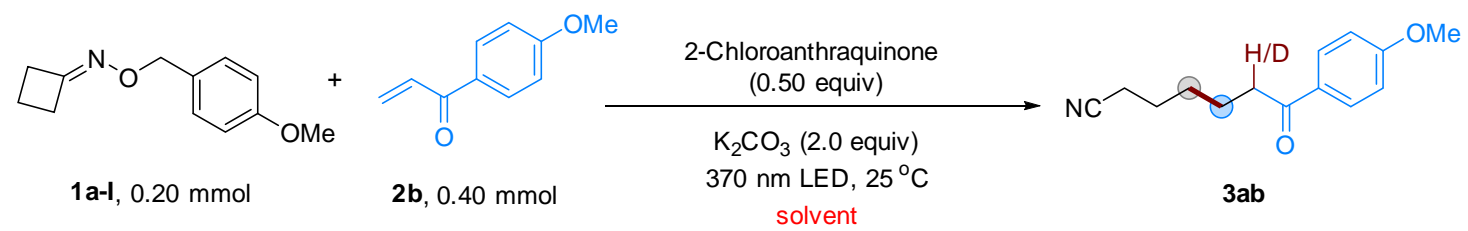

$$
\begin{aligned}
& \text { solvent } A \text { : acetone- } D_{6}(4.0 \mathrm{~mL}) \\
& \text { solvent } \mathrm{B}: \mathrm{D}_{2} \mathrm{O}(4.0 \text { equiv) + 2-butanone }(4.0 \mathrm{~mL}) \\
& \text { solvent } \mathrm{C}: \mathrm{D}_{2} \mathrm{O}\left(4.0 \text { equiv) + acetone- } \mathrm{D}_{6}(4.0 \mathrm{~mL})\right.
\end{aligned}
$$

In a dried sealed tube, 1a-I (41.06 mg, $0.20 \mathrm{mmol}), 2 \mathbf{b}(64.88 \mathrm{mg}, 0.40 \mathrm{mmol})$, 2-chloroanthraquinone (24.27 mg, 0.50 equiv) and $\mathrm{K}_{2} \mathrm{CO}_{3}(55.28 \mathrm{mg}, 2.0$ equiv) were dissolved in solvent $\mathrm{A}$ or $\mathrm{B}$ or $\mathrm{C}$ under a $\mathrm{Ar}$ atmosphere. Then, the resulting mixture was degassed via 'freeze-pump-thaw' procedure (3 times). After that, the solution was stirred at a distance of $\sim 5 \mathrm{~cm}$ from a $370 \mathrm{~nm}$ LED at room temperature about $12 \mathrm{~h}$ until the reaction was completed as monitored by TLC analysis. The crude product was purified by flash chromatography on silica gel (petroleum ether/ethylacetate 5:1) directly to give the desired product 3ab as a colorless oil.

Deuterated product 3ab was detected by ${ }^{1} \mathrm{H}-\mathrm{NMR}$, which suggests the hydrogen of product 3ab may come from the solvent $(40 \% \mathrm{D}$ when using solvent $\mathrm{A})$ and the trace amount of the system $(30 \% \mathrm{D}$ when using solvent $\mathrm{B}, 90 \% \mathrm{D}$ when using solvent $\mathrm{C})$.

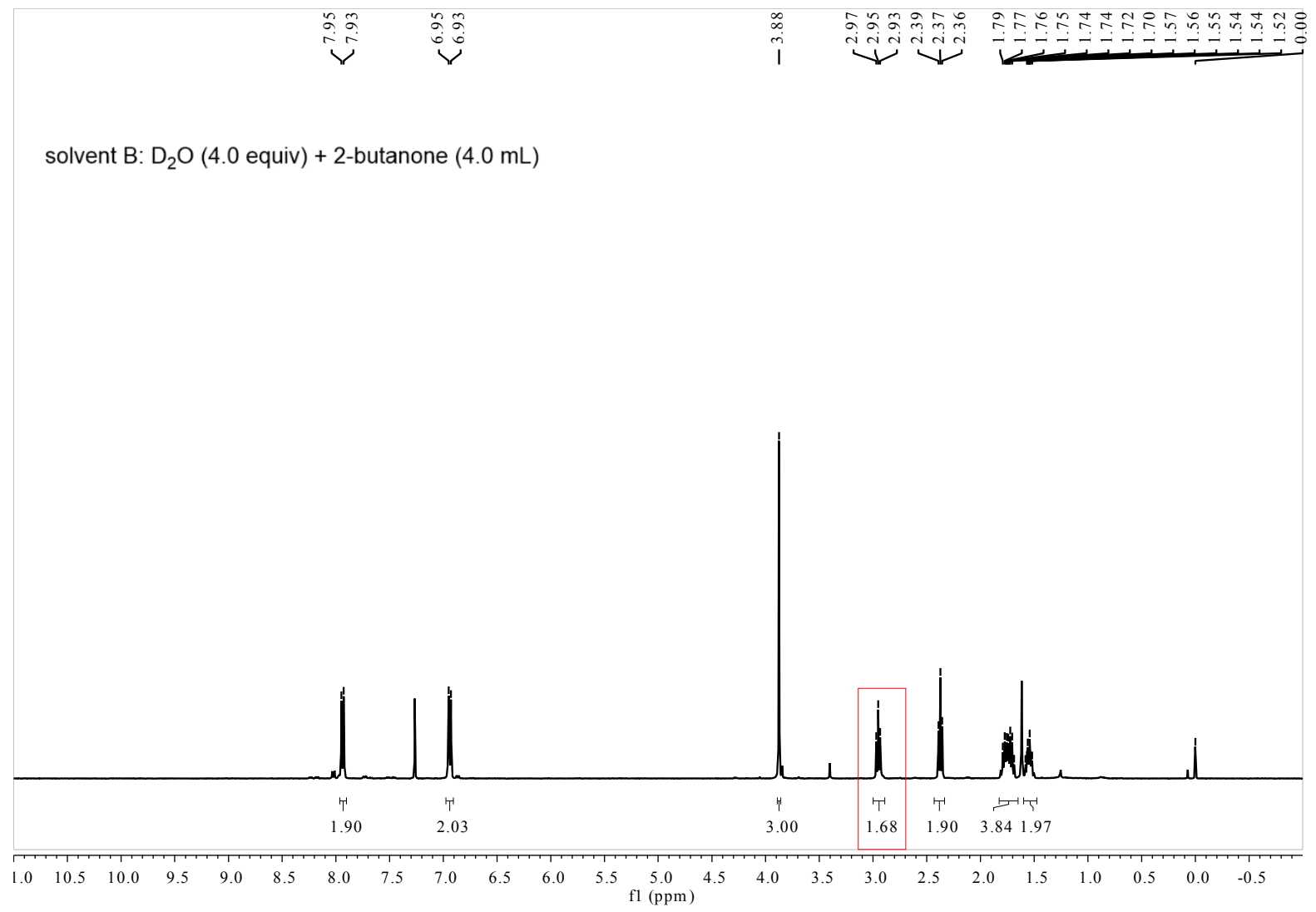




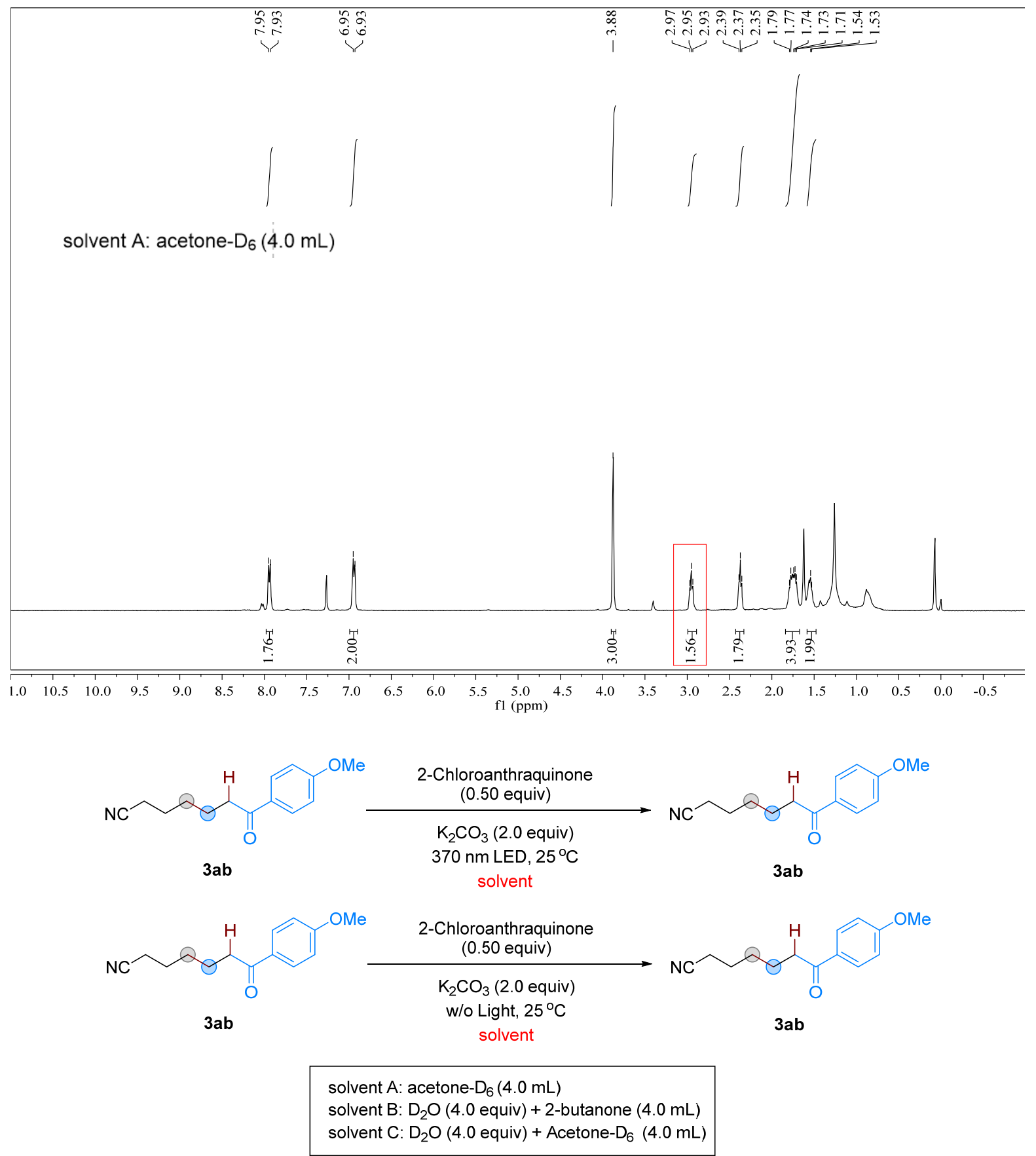

In four dried sealed tubes, 3ab (23.13 mg, $0.10 \mathrm{mmol})$, 2-chloroanthraquinone ( 0.50 equiv) and $\mathrm{K}_{2} \mathrm{CO}_{3}(2.0$ equiv) were dissolved in solvents protected by Ar under above four conditions. After that, the solution was stirred about $12 \mathrm{~h}$. Then, the solvent was concentrated in vacuo and the residual was characterized by ${ }^{1} \mathrm{H}$ NMR spectra.

No deuterated product $3 \mathrm{ab}$ was detected by ${ }^{1} \mathrm{H}-\mathrm{NMR}$, which suggests the hydrogen transfer process does not take place between products.

\subsection{Electrochemical Studies}

The oxidation potential of 1-Cl-AQ was $+1.9 \mathrm{~V}$ vs. FC/FC $\left(+2.3 \mathrm{~V}\right.$ vs. SCE) according to the literature. ${ }^{[1]}$

The cyclic voltammetry experiment was performed, and the oxidation potential of substrates with different protecting groups was determined as follows. The electrochemical measurements were carried out by a computer-controlled electrochemical analyzer. Cyclic voltammetry was performed in a three-electrode cell (volume $30 \mathrm{~mL}$; MeCN as solvent, $n-\mathrm{Bu}_{4} \mathrm{PF}_{6}(0.1 \mathrm{M})$ as the supporting electrolyte, $0.1 \mathrm{M}$ concentration of the tested compound) with glassy carbon 
(diameter $3 \mathrm{~mm}$ ) as the working electrode, Pt wire as the auxiliary electrode, and saturated calomel electrode as the reference electrode. The scan speed was $100 \mathrm{mV} \cdot \mathrm{s}^{-1}$.

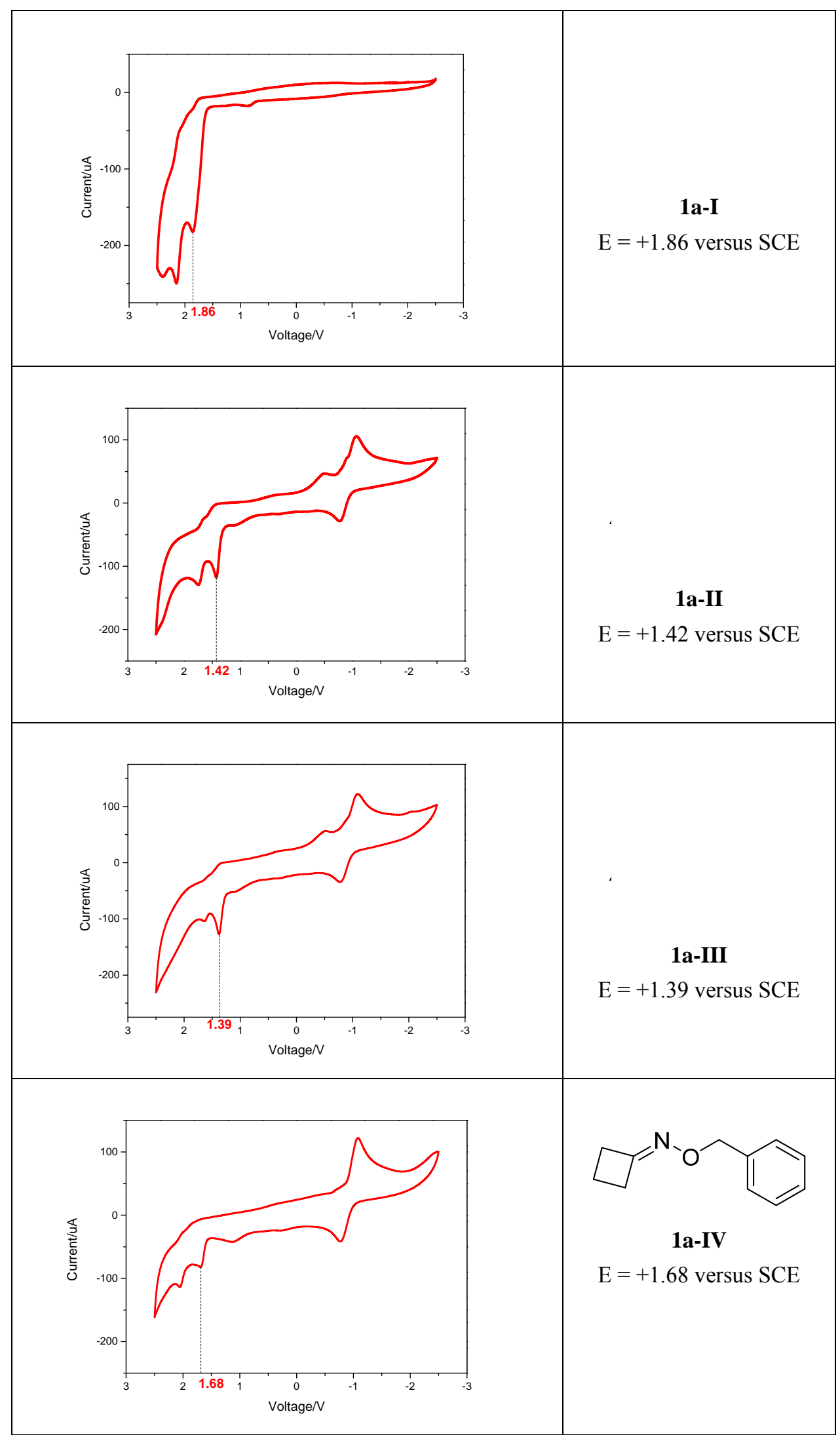




\subsection{Light On-Off Experiments}

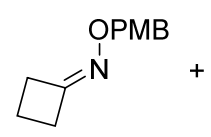

1a-I, $0.2 \mathrm{mmol}$

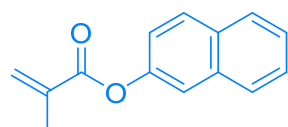

2e, $0.4 \mathrm{mmol}$

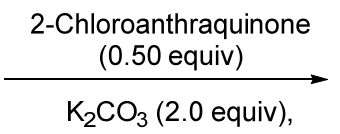

2-butanone $(4.0 \mathrm{~mL})$

$370 \mathrm{~nm}$ LED, $25^{\circ} \mathrm{C}$

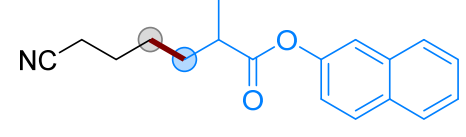

3ae

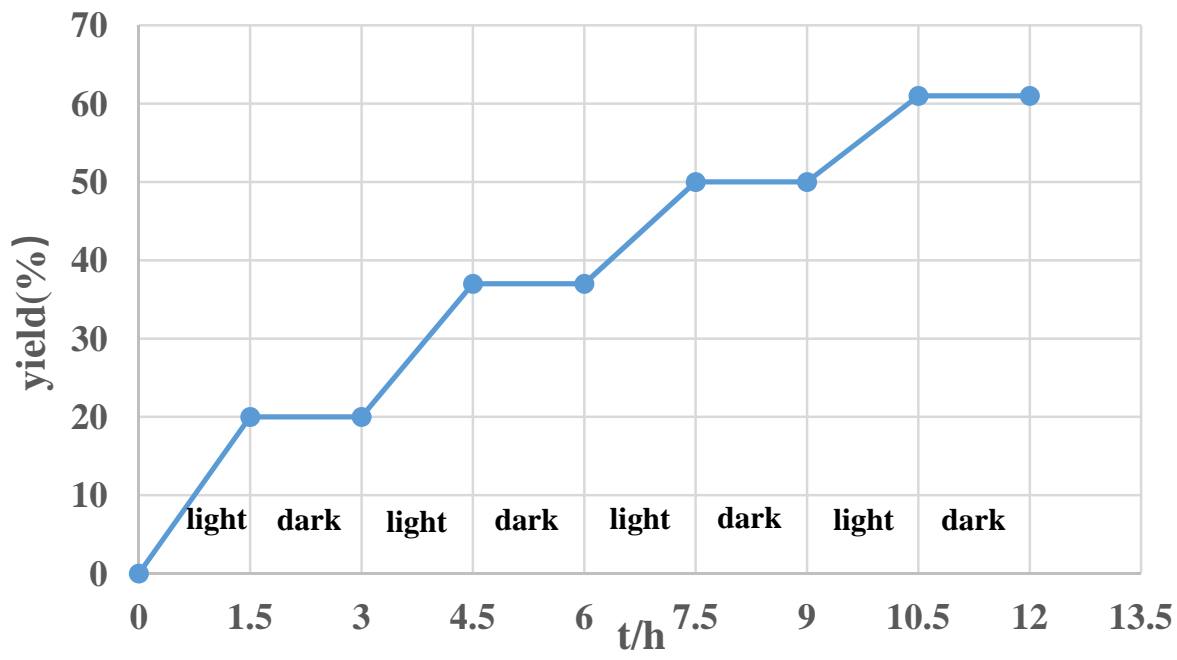

The yield of 3ae was determined by ${ }^{1} \mathrm{H}$ NMR using 1,3,5-trimethoxybenzene as an internal standard. The results reveal that a radical chain process is not the major reaction pathway, while it can not be completely ruled out at the current stage.

\subsection{Plausible Mechanism of PCET Pathway}

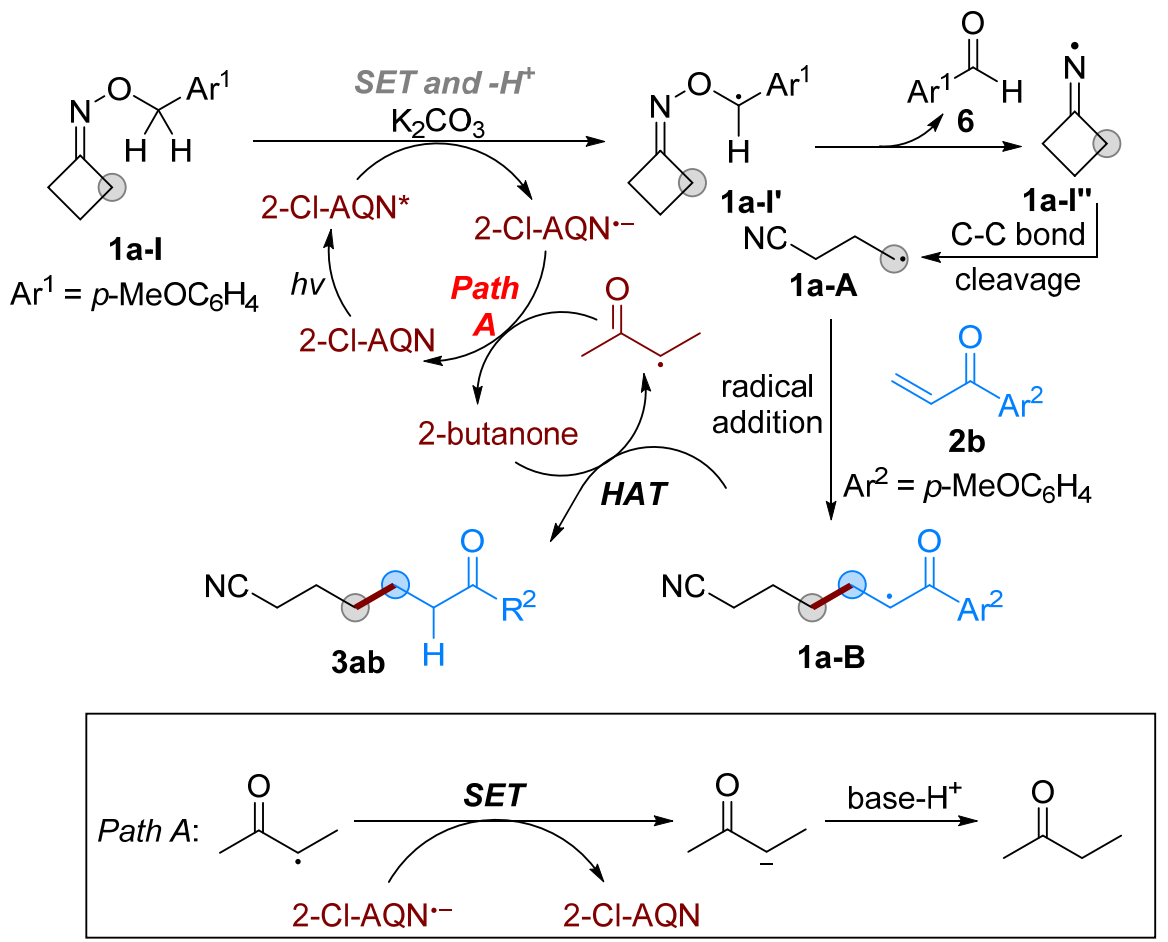




\section{Reference:}

[1] Usami, K., Yamaguchi, E., Tada, N., Itoh, A., Org. Lett. 2018, 20, 5714.

[2] Wang, P.-Z., Yu, X.-Y., Li, C.-Y., He, B.-Q., Chen, J.-R., Xiao, W.-J., Chem. Commun. 2018, 54, 9925.

[3] Yin, G.-Y., Wu, Y.-C., Liu, G.-S., J. Am. Chem. Soc. 2010, 34, 11978.

[4] Gu, Y.-R., Duan, X.-H., Lin, Y., Guo, L.-N., Org. Lett. 2017, 19, 5908. 


\section{The Spectra of Substrates and Products}

${ }^{1} \mathrm{H}$ NMR (400 MHz, CDCl 3$)$ and ${ }^{13} \mathrm{C}$ NMR (100 MHz, $\left.\mathrm{CDCl}_{3}\right)$ spectra of substrate 1a
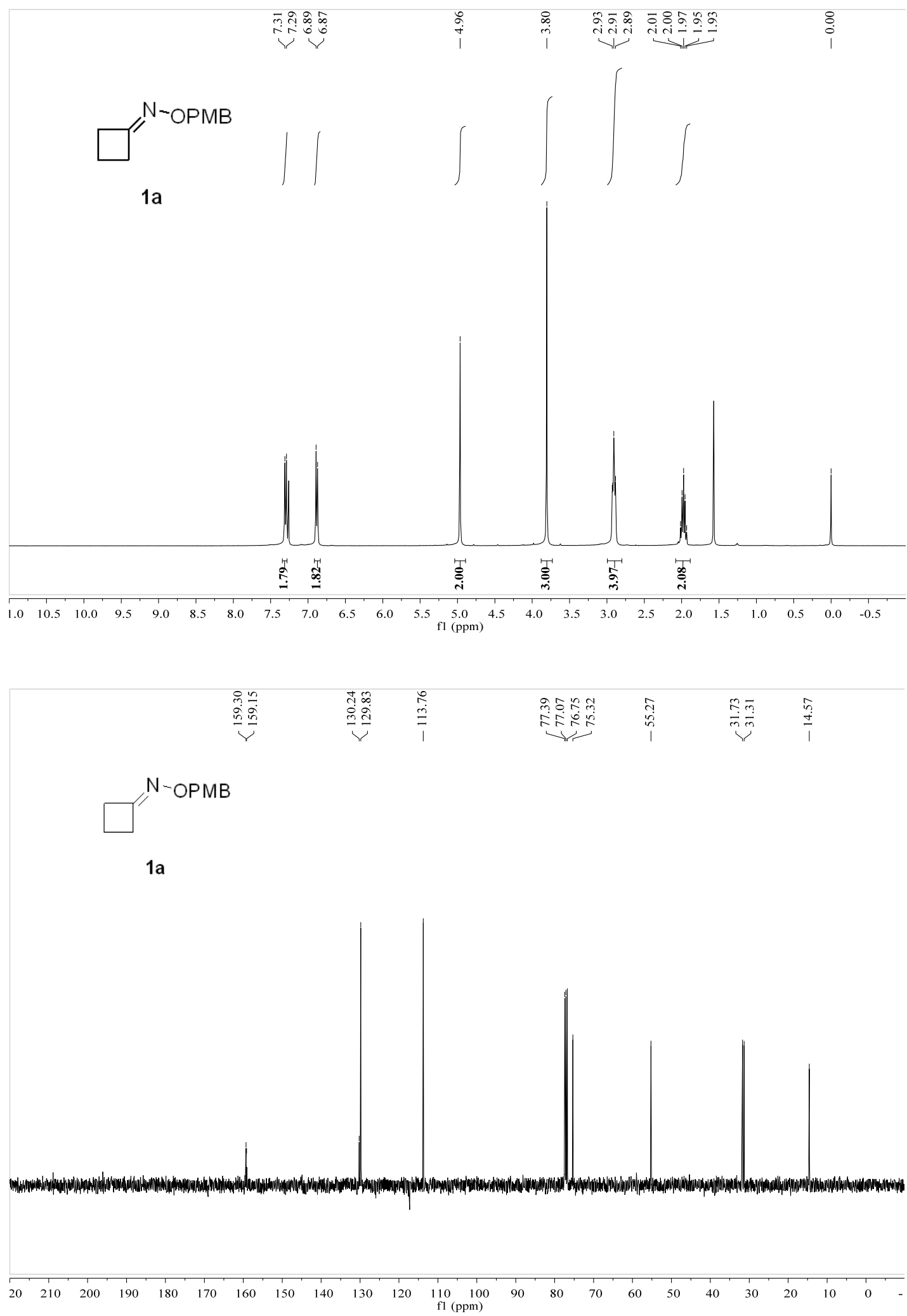
${ }^{1} \mathrm{H}$ NMR (400 MHz, $\left.\mathrm{CDCl}_{3}\right)$ and ${ }^{13} \mathrm{C}$ NMR (100 MHz, $\left.\mathrm{CDCl}_{3}\right)$ spectra of substrate $1 \mathrm{~b}$
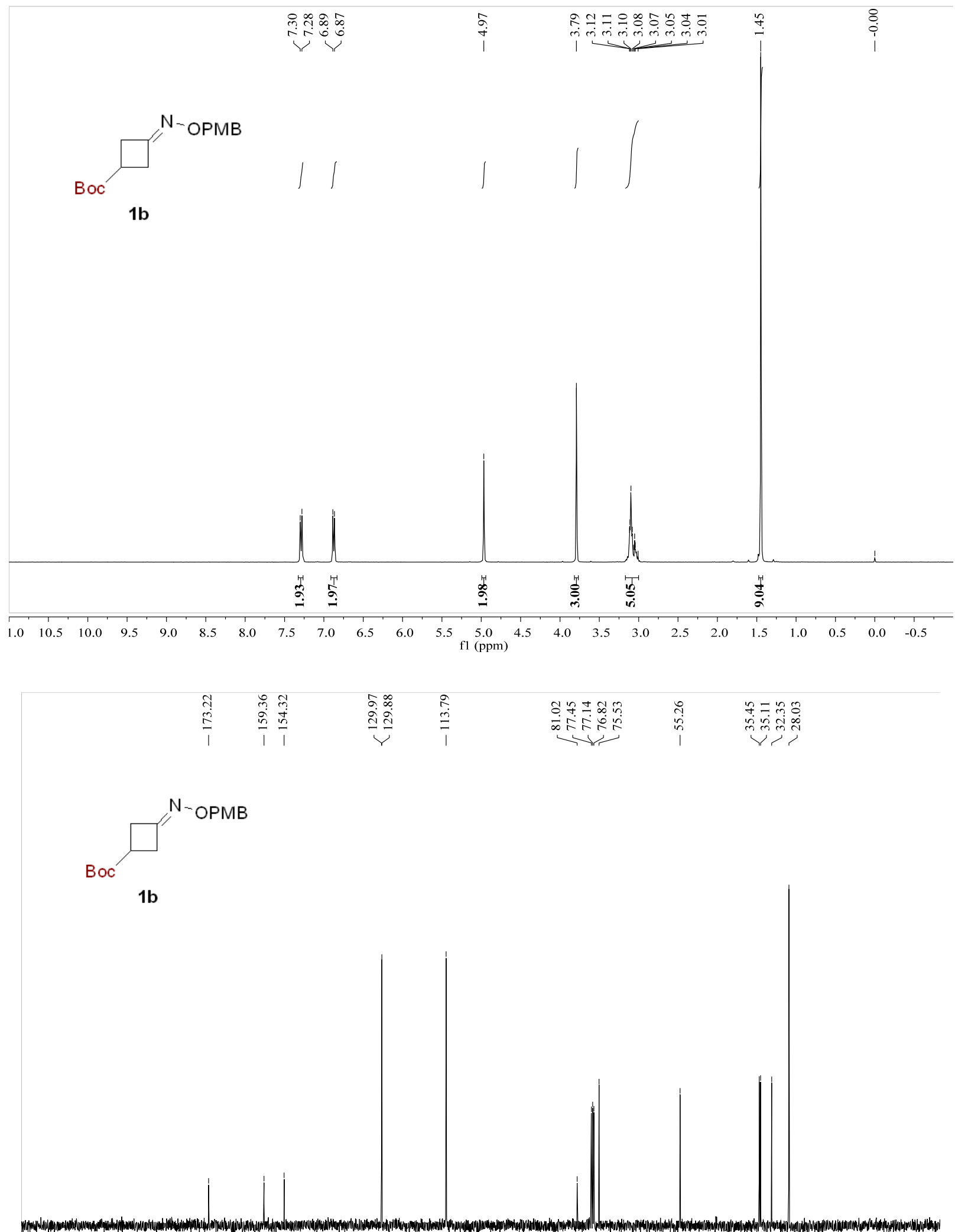

$\begin{array}{llllllllllllllllllllllllllllllll}20 & 210 & 200 & 190 & 180 & 170 & 160 & 150 & 140 & 130 & 120 & 110 & 100 & 90 & 80 & 70 & 60 & 50 & 40 & 30 & 20 & 10 & 0 & -\end{array}$ 
${ }^{1} \mathrm{H}$ NMR (400 MHz, $\left.\mathrm{CDCl}_{3}\right)$ and ${ }^{13} \mathrm{C}$ NMR (100 MHz, $\left.\mathrm{CDCl}_{3}\right)$ spectra of substrate 1c
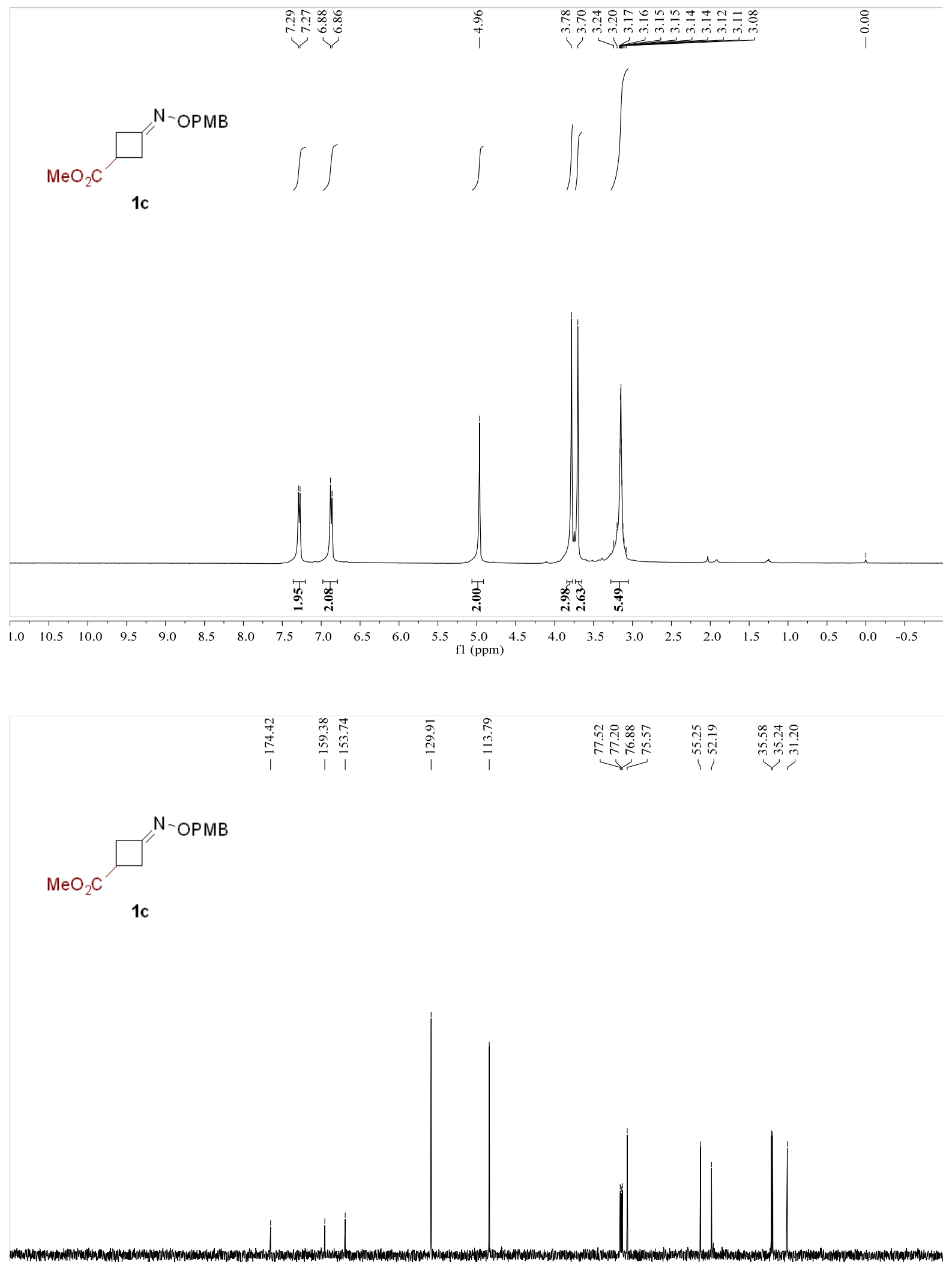

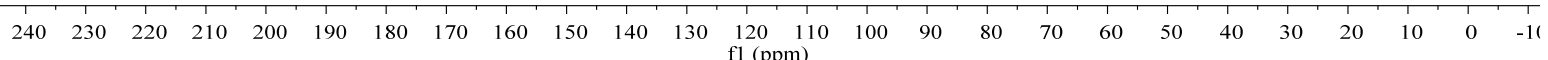


${ }^{1} \mathrm{H}$ NMR (400 MHz, $\left.\mathrm{CDCl}_{3}\right)$ and ${ }^{13} \mathrm{C}$ NMR (100 MHz, $\left.\mathrm{CDCl}_{3}\right)$ spectra of substrate 1d
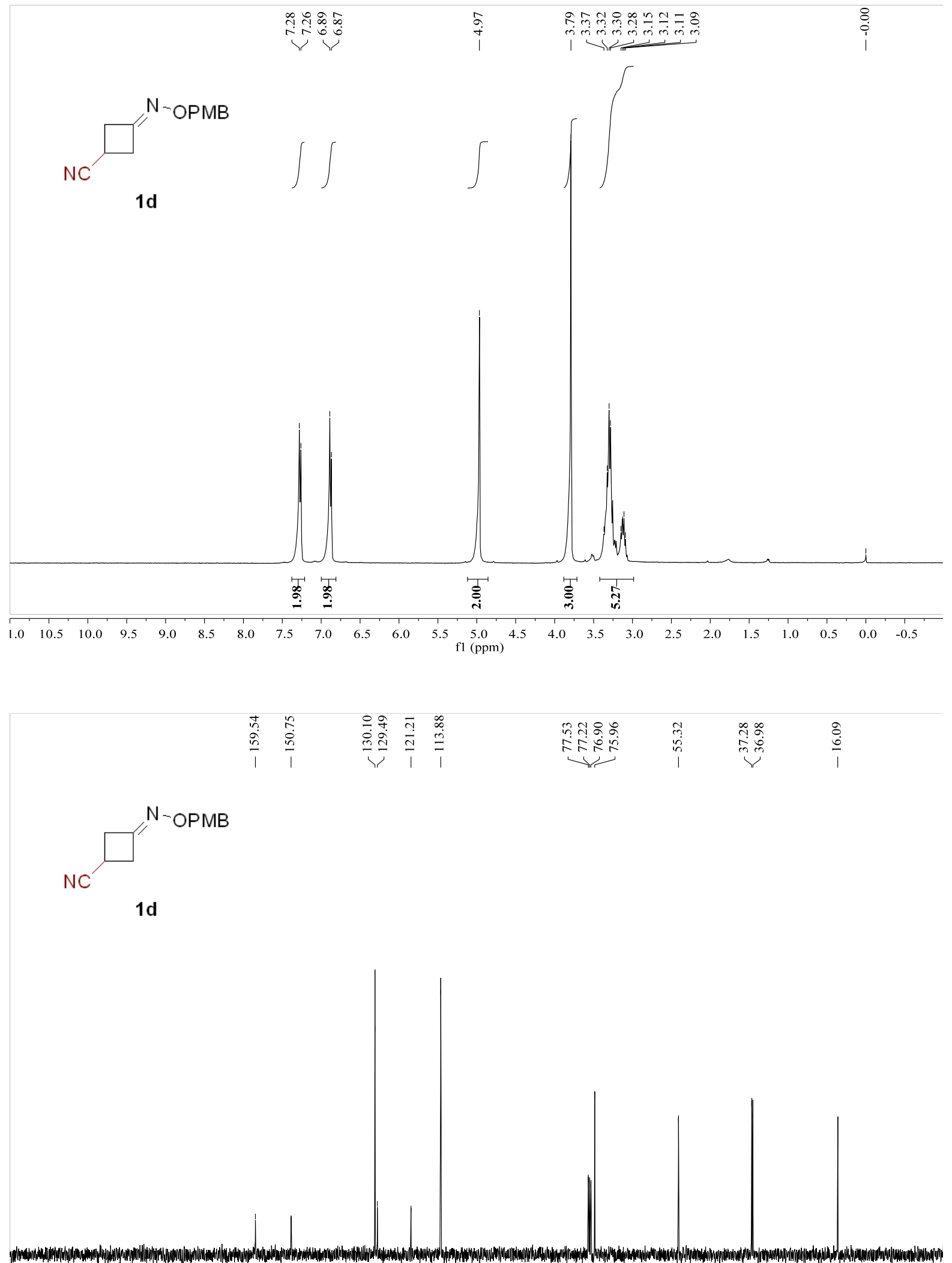

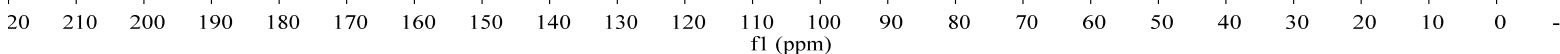


${ }^{1} \mathrm{H}$ NMR (400 MHz, $\left.\mathrm{CDCl}_{3}\right)$ and ${ }^{13} \mathrm{C}$ NMR (100 MHz, $\left.\mathrm{CDCl}_{3}\right)$ spectra of substrate 1e
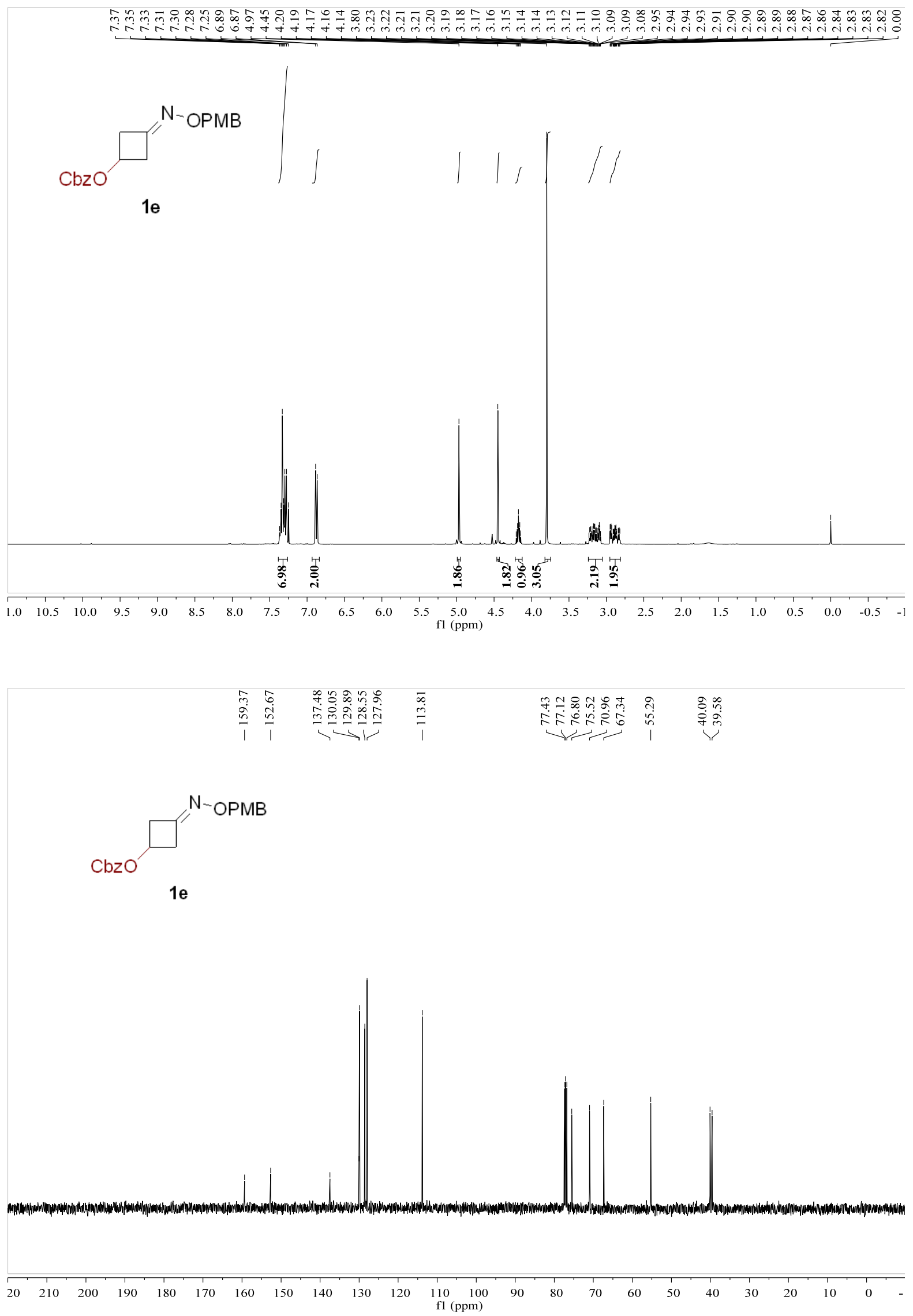
${ }^{1} \mathrm{H}$ NMR (400 MHz, $\left.\mathrm{CDCl}_{3}\right)$ and ${ }^{13} \mathrm{C}$ NMR (100 MHz, $\left.\mathrm{CDCl}_{3}\right)$ spectra of substrate if
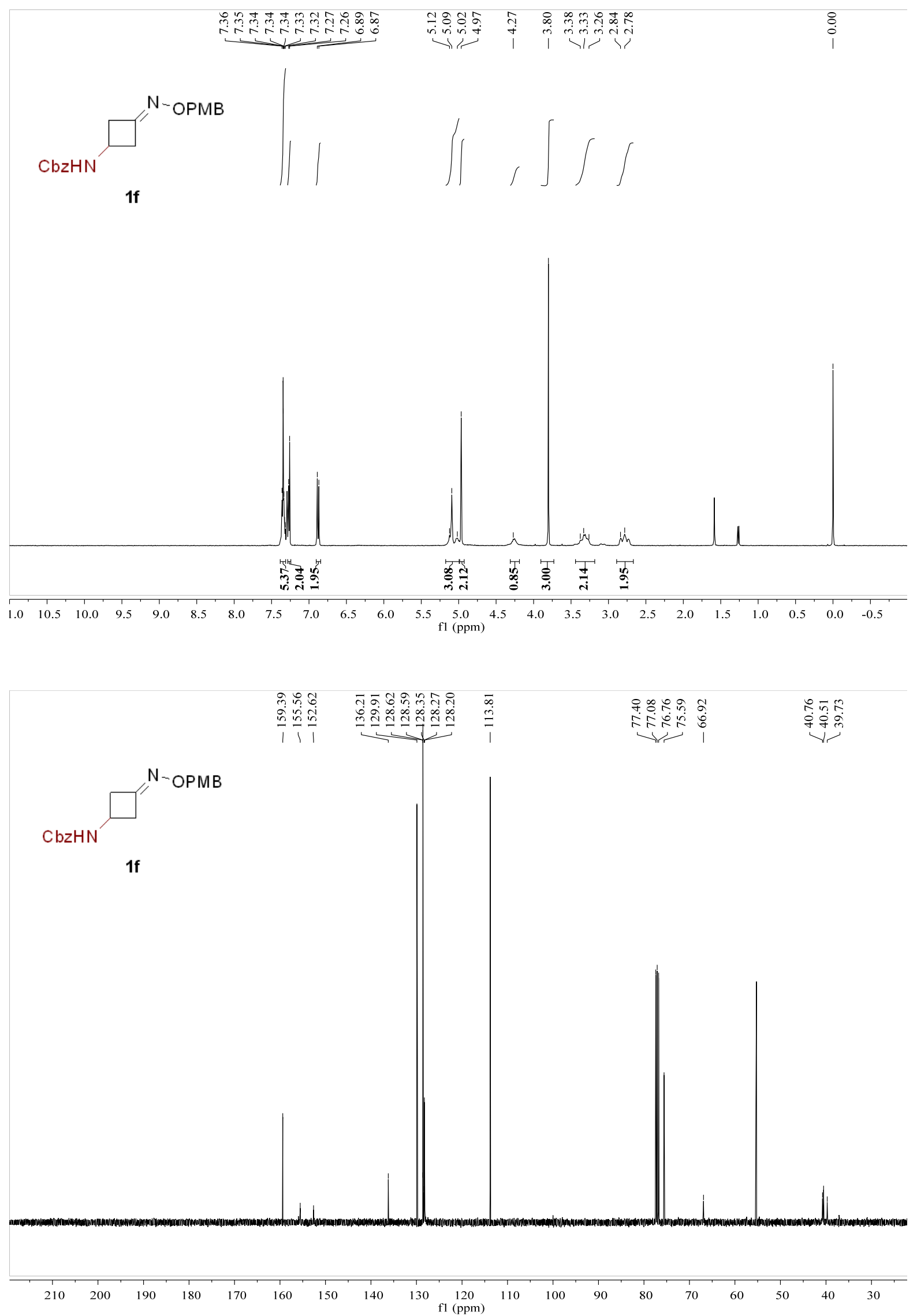
${ }^{1} \mathrm{H}$ NMR (400 MHz, $\left.\mathrm{CDCl}_{3}\right)$ and ${ }^{13} \mathrm{C}$ NMR (100 MHz, $\left.\mathrm{CDCl}_{3}\right)$ spectra of substrate $1 \mathrm{~g}$
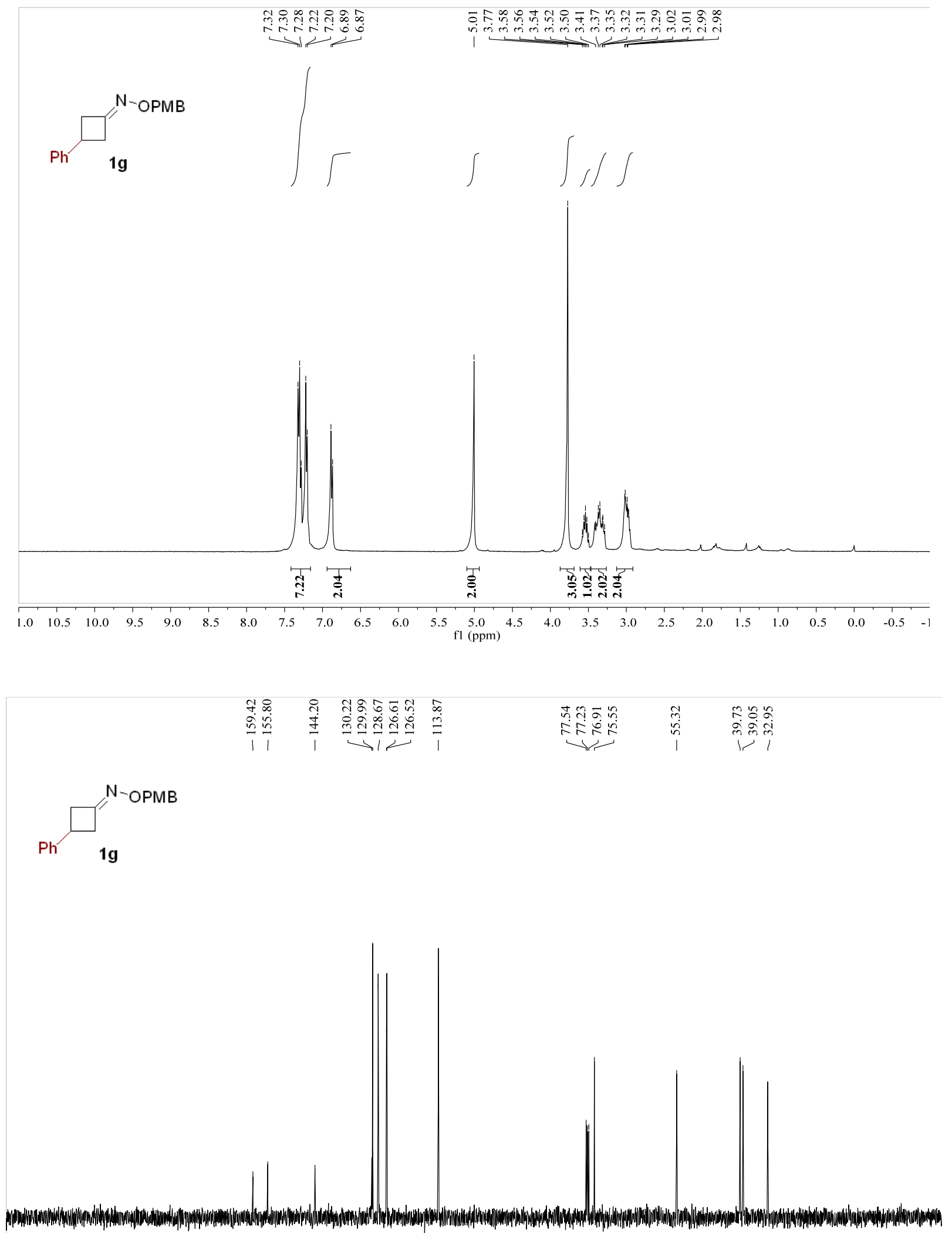

$\begin{array}{lllllllllllllllllllllllll}20 & 210 & 200 & 190 & 180 & 170 & 160 & 150 & 140 & 130 & 120 & \begin{array}{c}110 \\ \mathrm{f} 1(\mathrm{ppm})\end{array} & 100 & 80 & 70 & 60 & 50 & 40 & 30 & 20 & 10 & 0 & -\end{array}$ 
${ }^{1} \mathrm{H}$ NMR (400 MHz, CDCl $)$ and ${ }^{13} \mathrm{C}$ NMR (100 MHz, $\left.\mathrm{CDCl}_{3}\right)$ spectra of substrate $1 \mathrm{~h}$
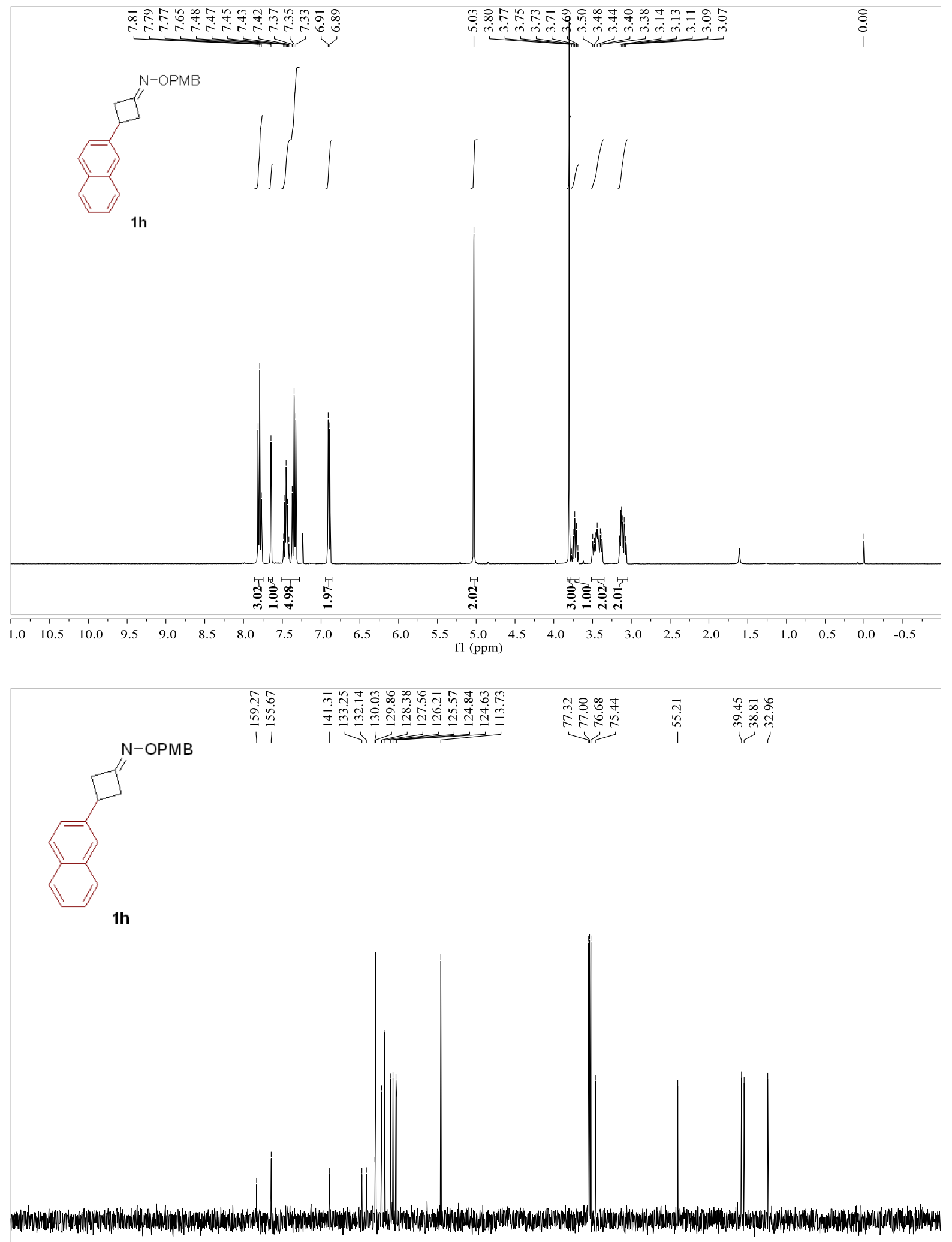

$\begin{array}{lllllllllllll}20 & 210 & 200 & 190 & 180 & 170 & 160 & 150 & 140 & 130 & 120 & 110 & 100\end{array}$ 
${ }^{1} \mathrm{H}$ NMR (400 MHz, $\left.\mathrm{CDCl}_{3}\right)$ and ${ }^{13} \mathrm{C}$ NMR (100 MHz, $\left.\mathrm{CDCl}_{3}\right)$ spectra of substrate 1i
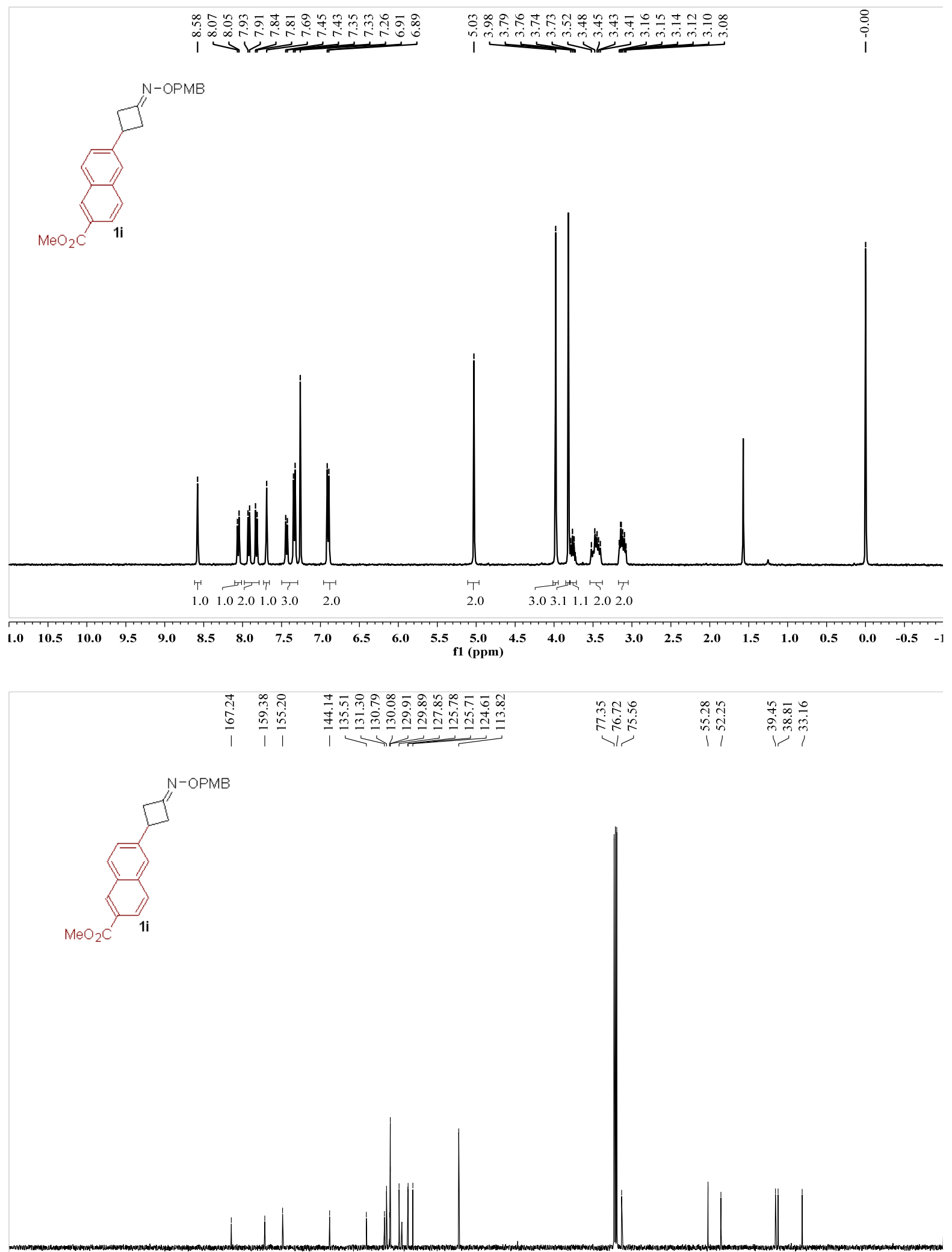

$\begin{array}{llllllllllllllllllllllll}1 & 210 & 200 & 190 & 180 & 170 & 160 & 150 & 140 & 130 & 120 & 110 & 100 & 90 & 80 & 70 & 60 & 50 & 40 & 30 & 20 & 10\end{array}$ 
${ }^{1} \mathrm{H}$ NMR (400 MHz, $\left.\mathrm{CDCl}_{3}\right)$ and ${ }^{13} \mathrm{C}$ NMR (100 MHz, CDCl$)$ spectra of substrate $1 \mathrm{j}$
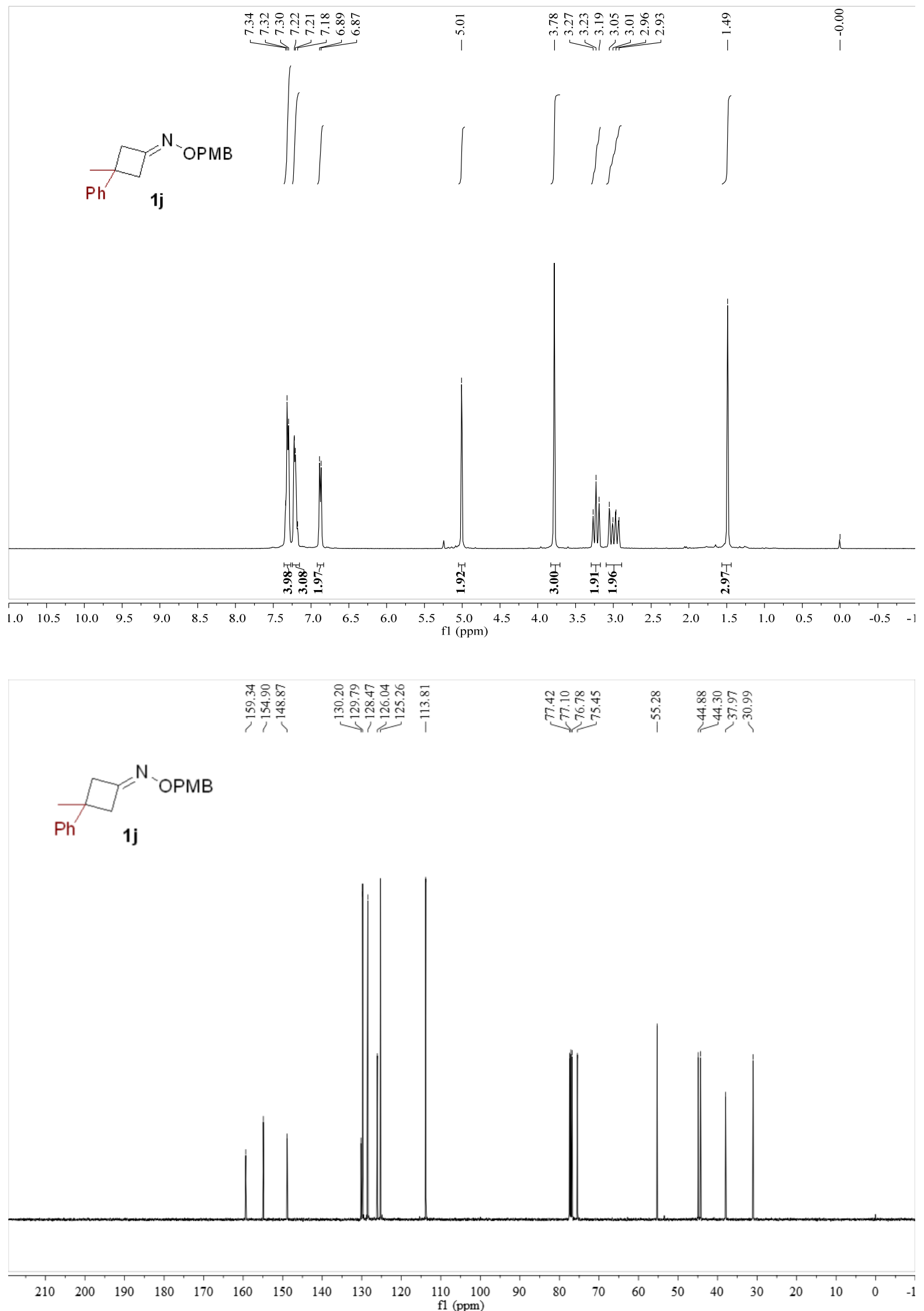
${ }^{1} \mathrm{H}$ NMR (400 MHz, CDCl $)$ and ${ }^{13} \mathrm{C}$ NMR $\left(100 \mathrm{MHz}, \mathrm{CDCl}_{3}\right)$ spectra of substrate $1 \mathrm{k}$

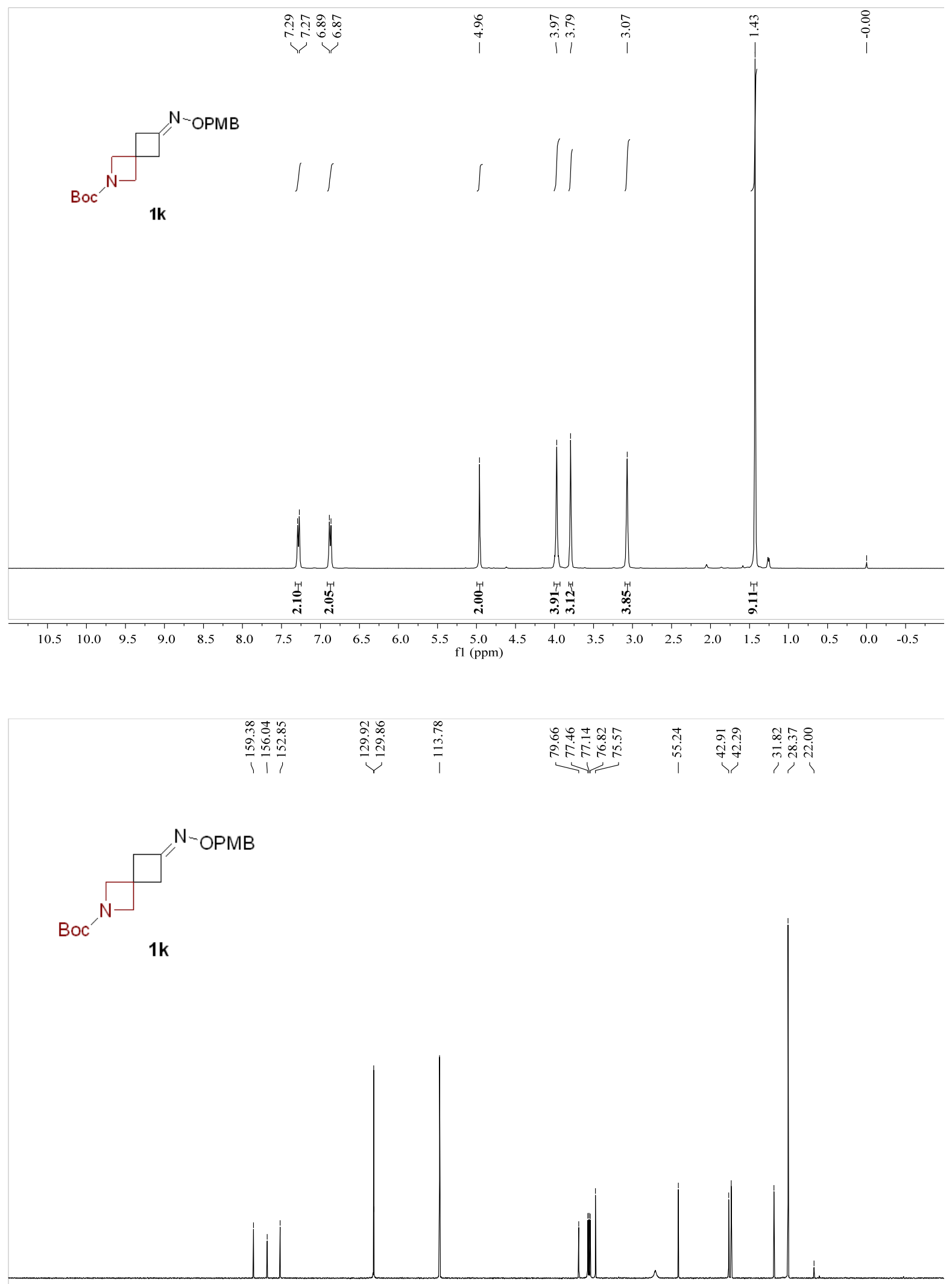

$\begin{array}{llllllllllllllllllllllllll}210 & 200 & 190 & 180 & 170 & 160 & 150 & 140 & 130 & 120 & 110 & 100 & 90 & 80 & 70 & 60 & 50 & 40 & 30 & 20 & 10 & 0 & -\end{array}$ 
${ }^{1} \mathrm{H}$ NMR (400 MHz, $\left.\mathrm{CDCl}_{3}\right)$ and ${ }^{13} \mathrm{C}$ NMR (100 MHz, $\left.\mathrm{CDCl}_{3}\right)$ spectra of substrate $1 \mathrm{l}$
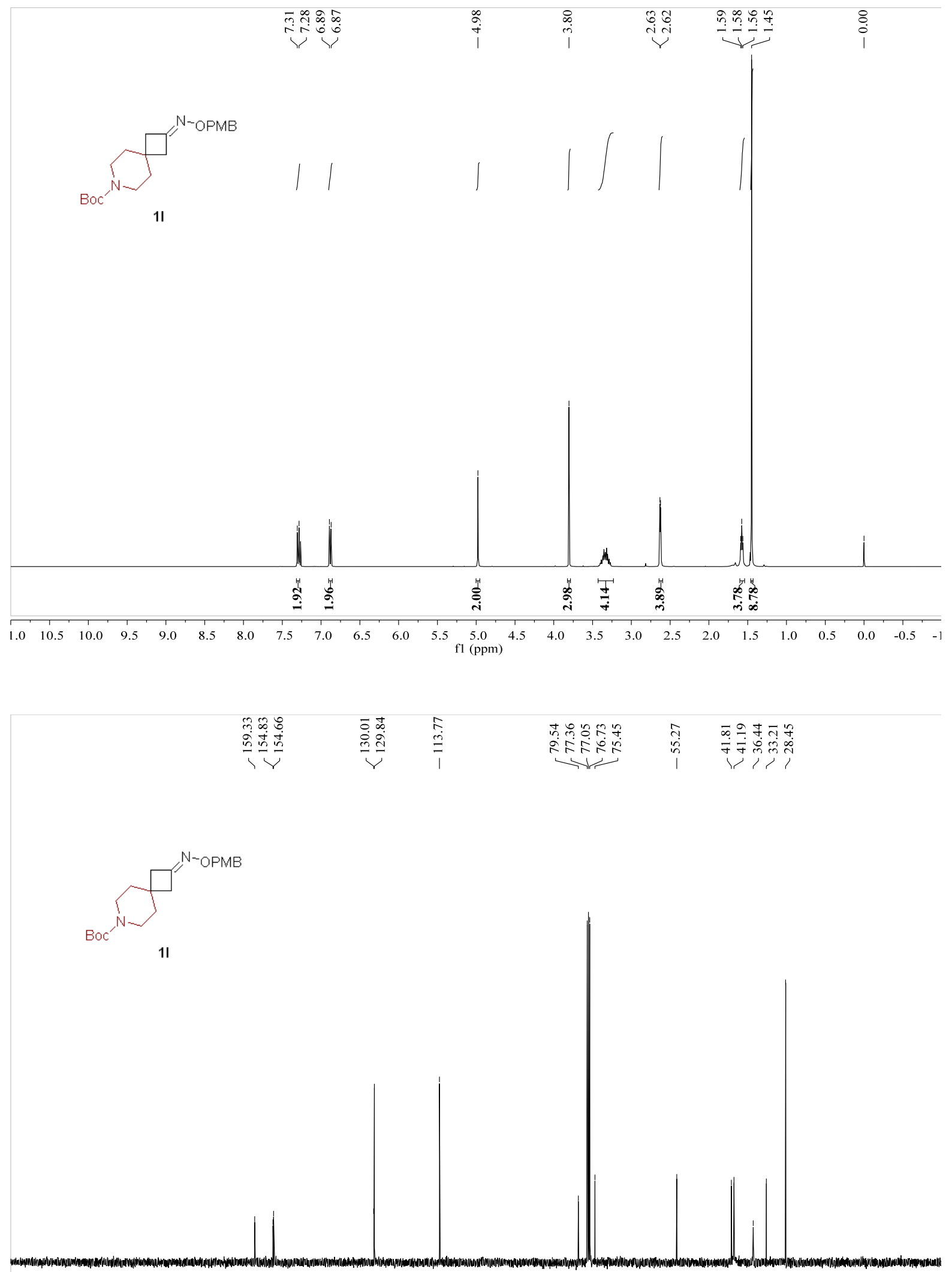

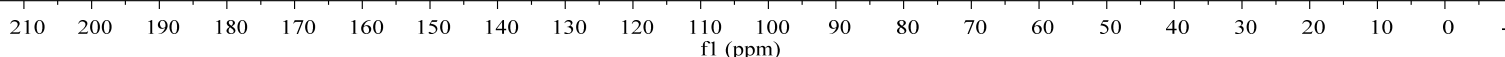


${ }^{1} \mathrm{H}$ NMR (400 MHz, $\left.\mathrm{CDCl}_{3}\right)$ and ${ }^{13} \mathrm{C}$ NMR $\left(100 \mathrm{MHz}, \mathrm{CDCl}_{3}\right)$ spectra of substrate $1 \mathrm{~m}$

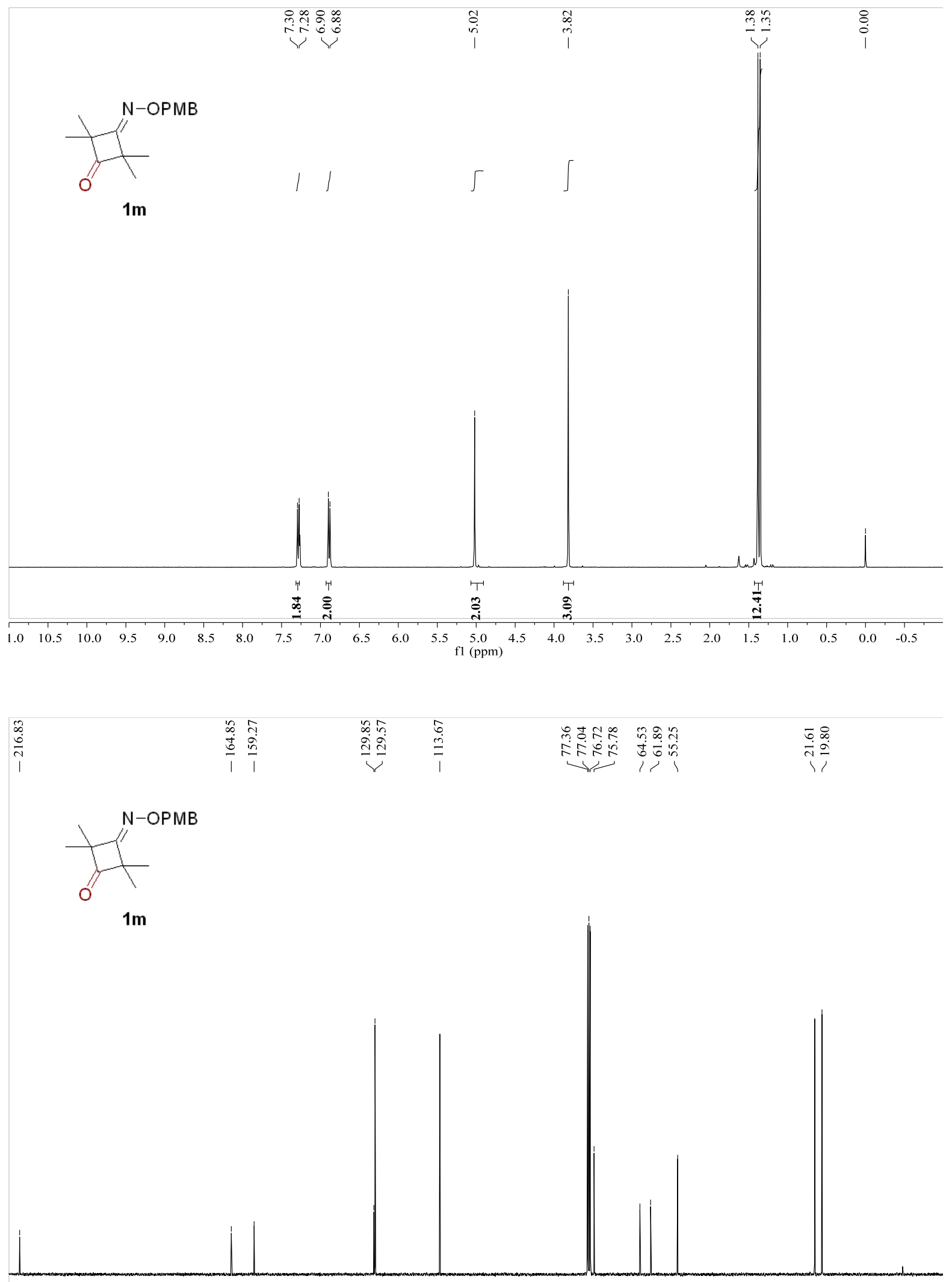

$\begin{array}{llllllllllllllllllllllll}210 & 200 & 190 & 180 & 170 & 160 & 150 & 140 & 130 & 120 & \begin{array}{c}110 \\ \mathrm{fl}(\mathrm{ppm})\end{array} & 100 & 80 & 70 & 60 & 50 & 40 & 30 & 20 & 10 & 0 & -\end{array}$ 
${ }^{1} \mathrm{H}$ NMR (400 MHz, $\left.\mathrm{CDCl}_{3}\right)$ and ${ }^{13} \mathrm{C}$ NMR (100 $\left.\mathrm{MHz}, \mathrm{CDCl}_{3}\right)$ spectra of substrate $1 \mathrm{n}$
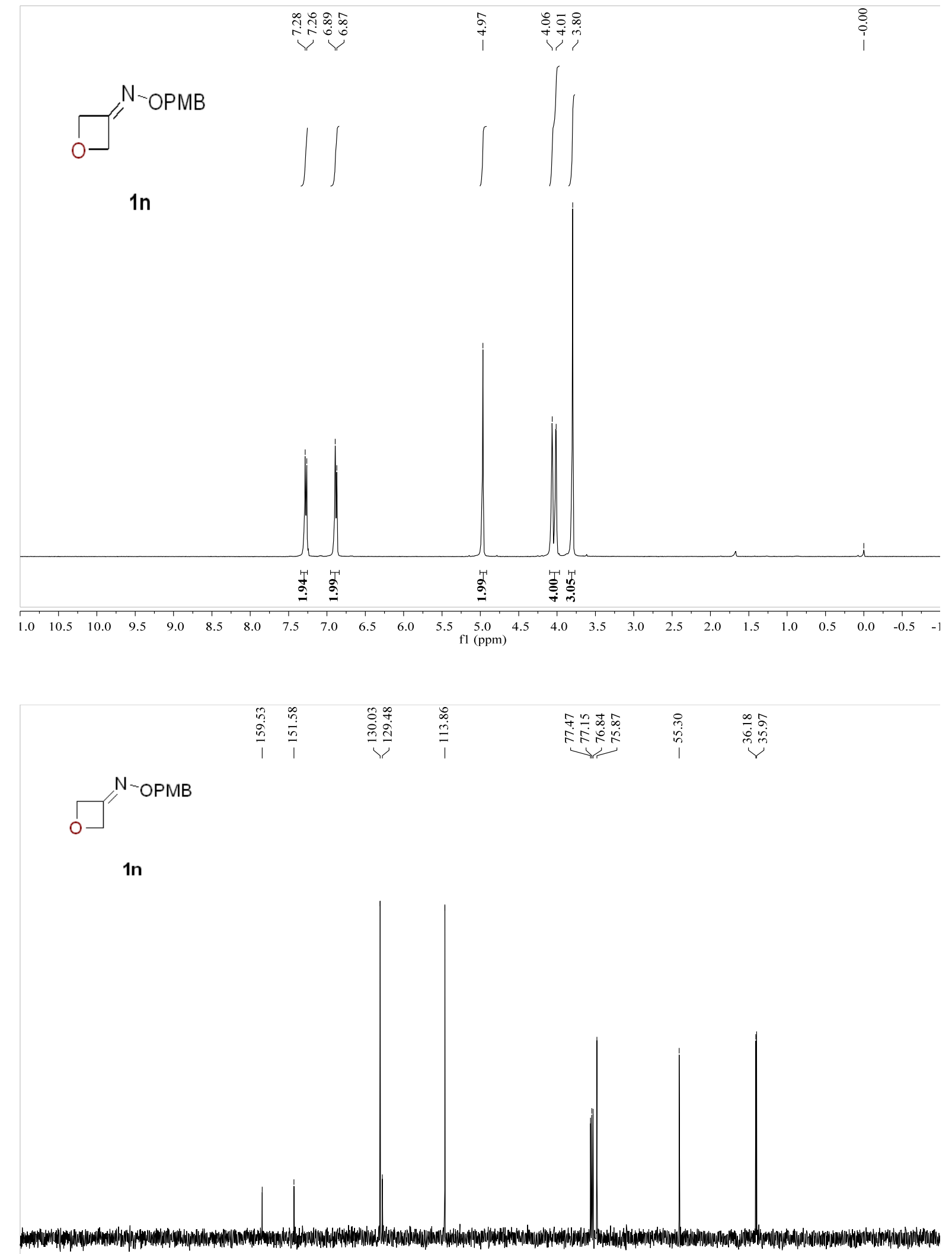

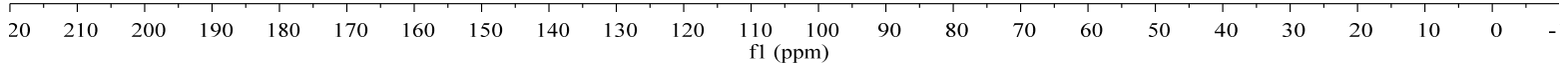


${ }^{1} \mathrm{H}$ NMR (400 MHz, $\left.\mathrm{CDCl}_{3}\right)$ and ${ }^{13} \mathrm{C}$ NMR (100 MHz, $\left.\mathrm{CDCl}_{3}\right)$ spectra of substrate 10

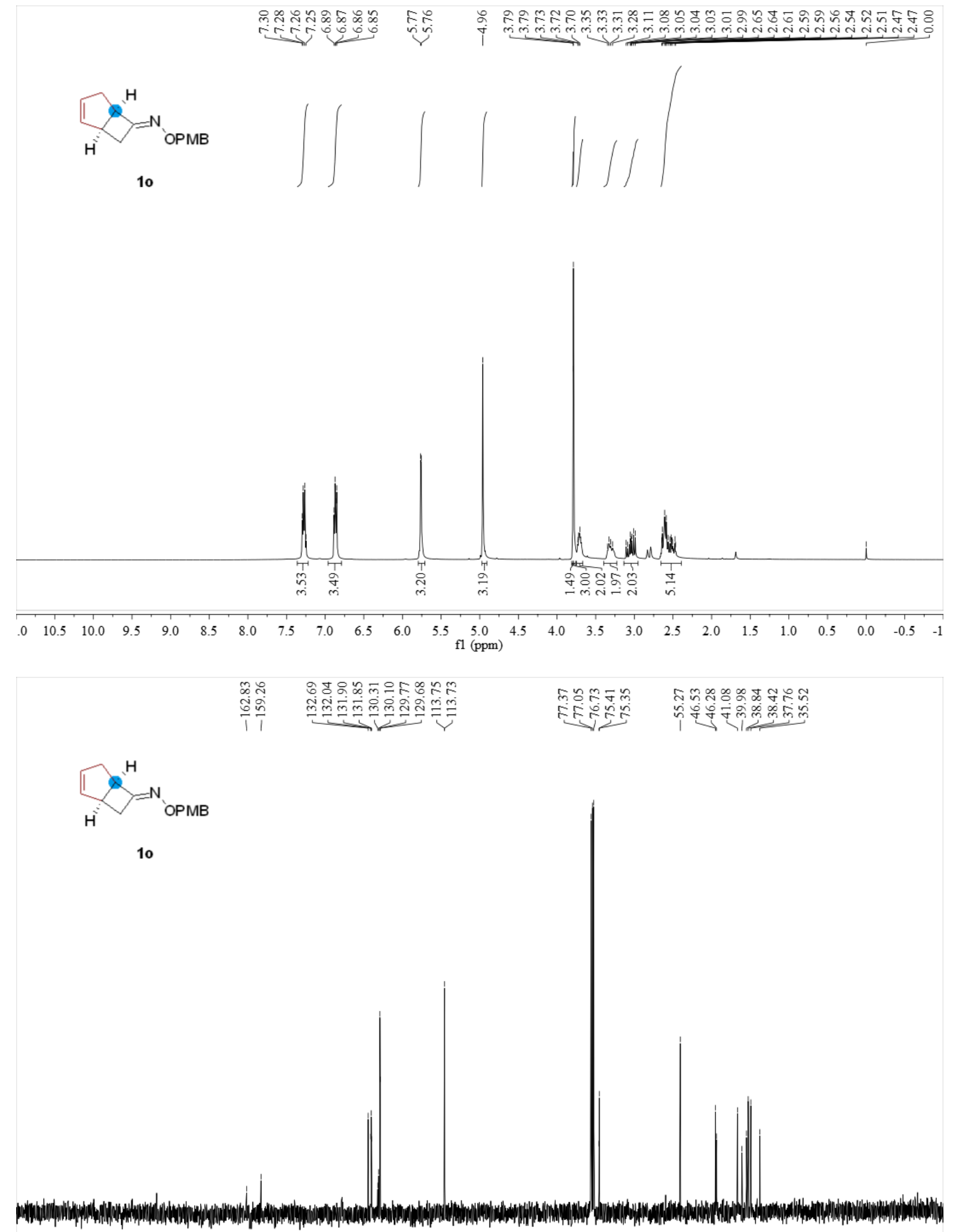

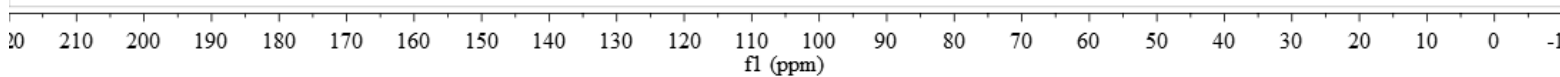


${ }^{1} \mathrm{H}$ NMR (400 MHz, CDCl $)$ and ${ }^{13} \mathrm{C}$ NMR (100 MHz, $\left.\mathrm{CDCl}_{3}\right)$ spectra of substrate 1p
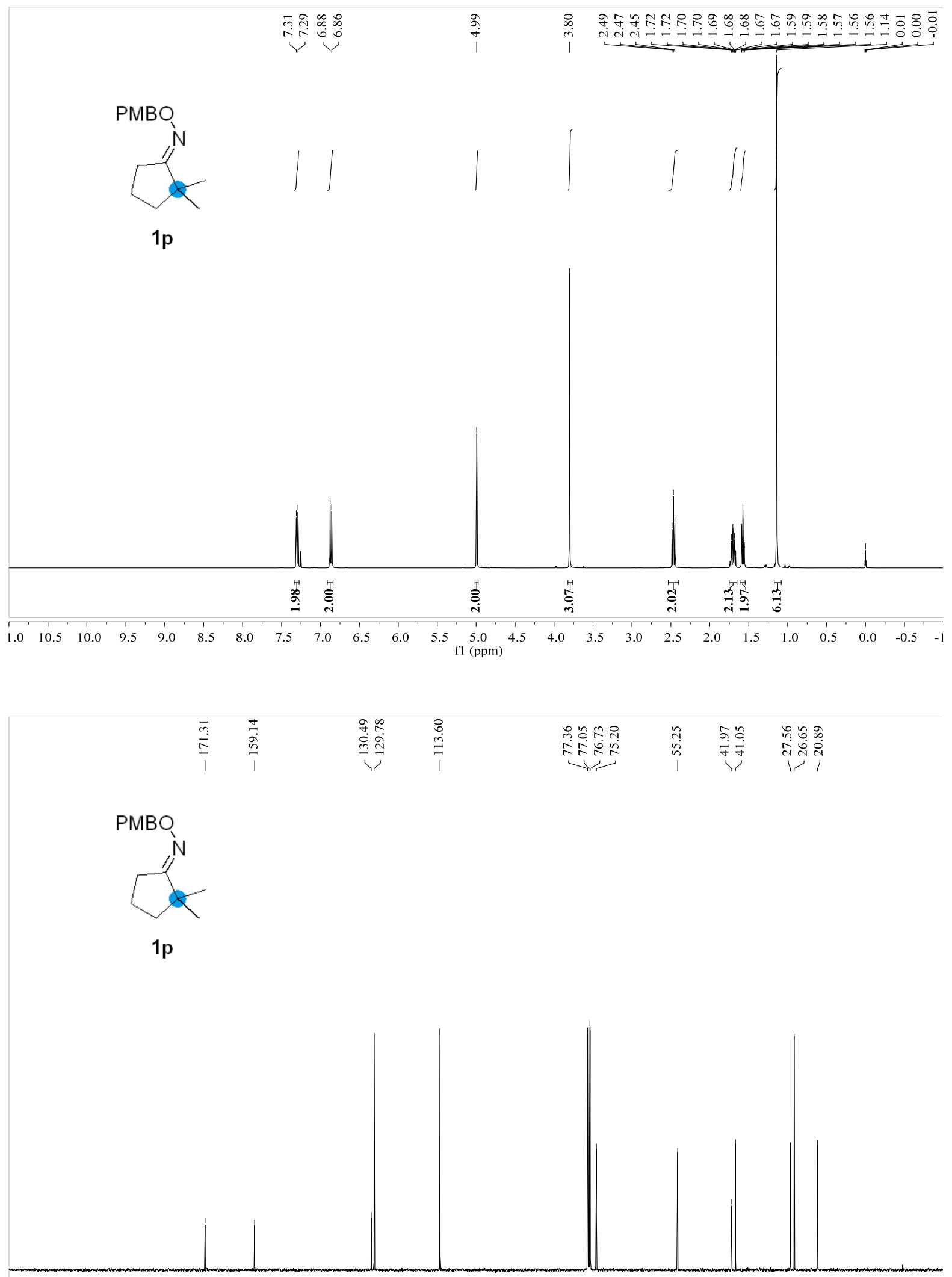

$\begin{array}{llllllllllllllllllllllllll}210 & 200 & 190 & 180 & 170 & 160 & 150 & 140 & 130 & 120 & 110 & 100 & 90 & 80 & 70 & 60 & 50 & 40 & 30 & 20 & 10 & 0 & -\end{array}$ 
${ }^{1} \mathrm{H}$ NMR (400 MHz, $\left.\mathrm{CDCl}_{3}\right)$ and ${ }^{13} \mathrm{C}$ NMR (100 MHz, $\left.\mathrm{CDCl}_{3}\right)$ spectra of substrate 1q

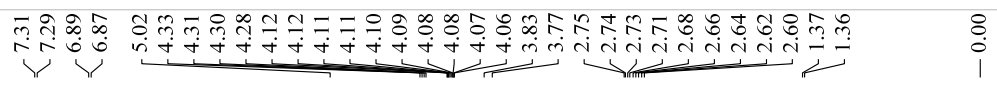

PMBO<smiles>CC1=C(C)OCC1</smiles><smiles>C1=CC2C=C1C2</smiles>

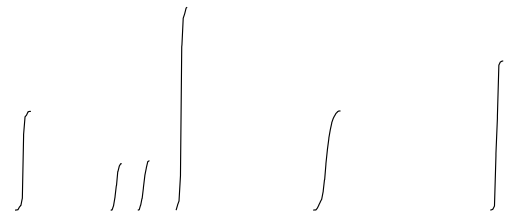

1q

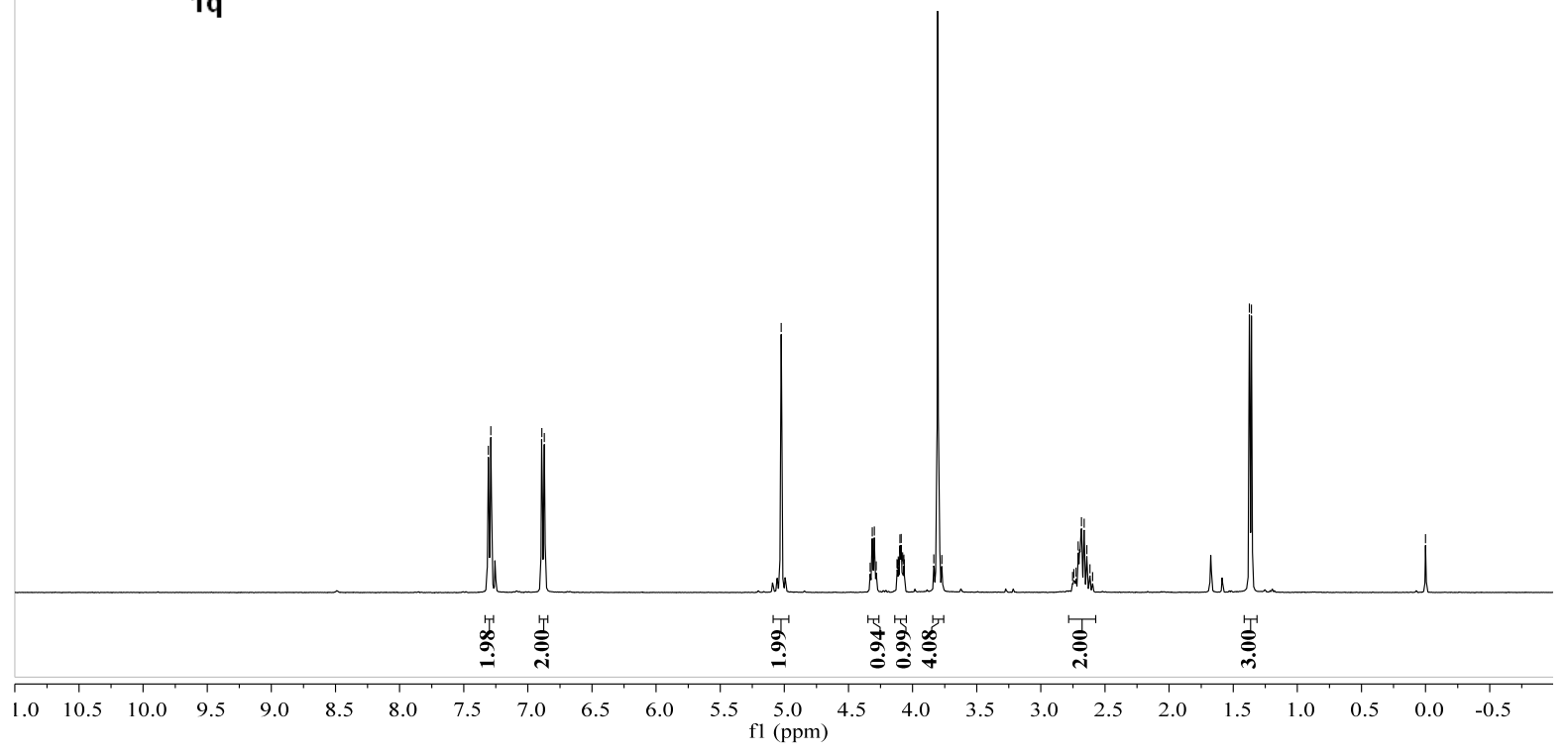

PMBO

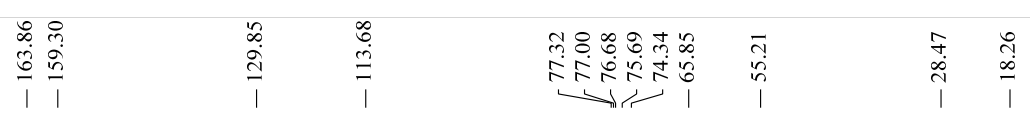

$\int_{0}^{N}$

19

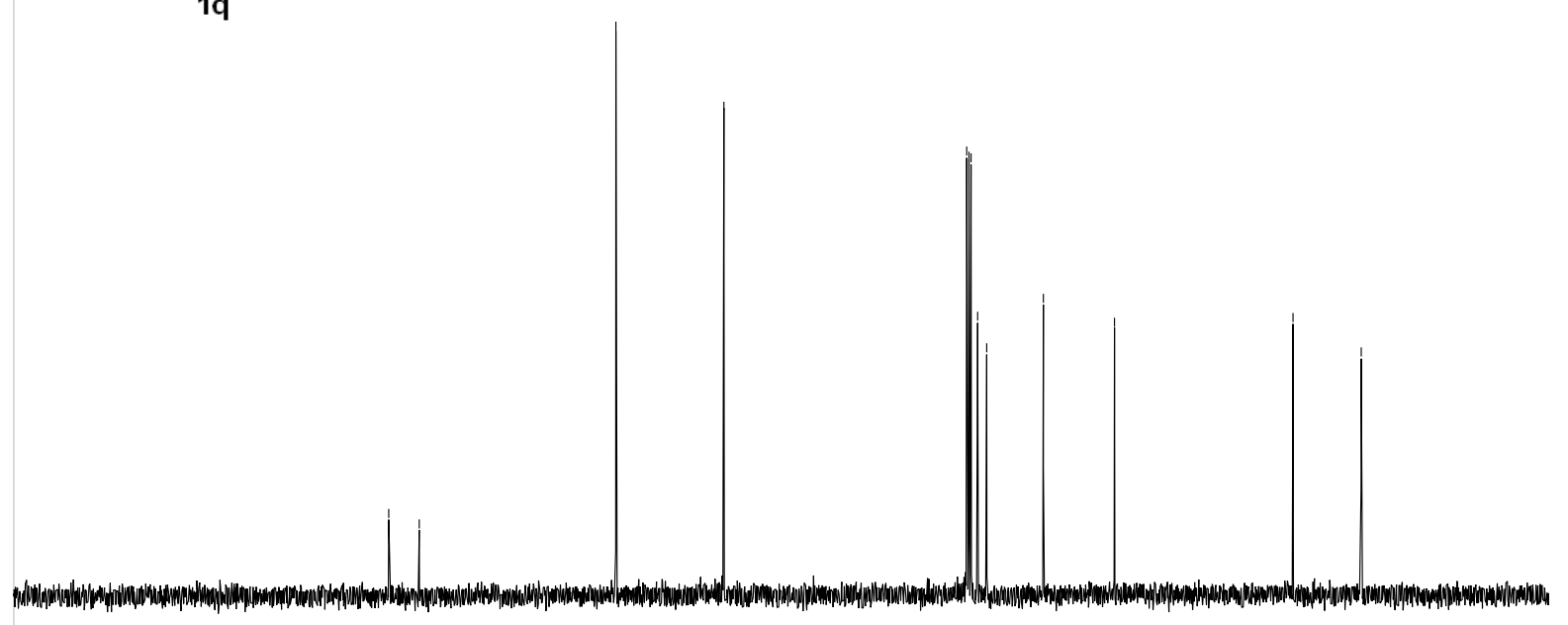

$\begin{array}{lllllllllllllllllllllll}20 & 210 & 200 & 190 & 180 & 170 & 160 & 150 & 140 & 130 & 120 & \begin{array}{c}110 \\ \mathrm{fl}(\mathrm{ppm})\end{array} & 90 & 80 & 70 & 60 & 50 & 40 & 30 & 20 & 10 & 0 & -\end{array}$ 
${ }^{1} \mathrm{H}$ NMR (400 MHz, $\left.\mathrm{CDCl}_{3}\right)$ and ${ }^{13} \mathrm{C}$ NMR (100 MHz, $\left.\mathrm{CDCl}_{3}\right)$ spectra of product 3ab
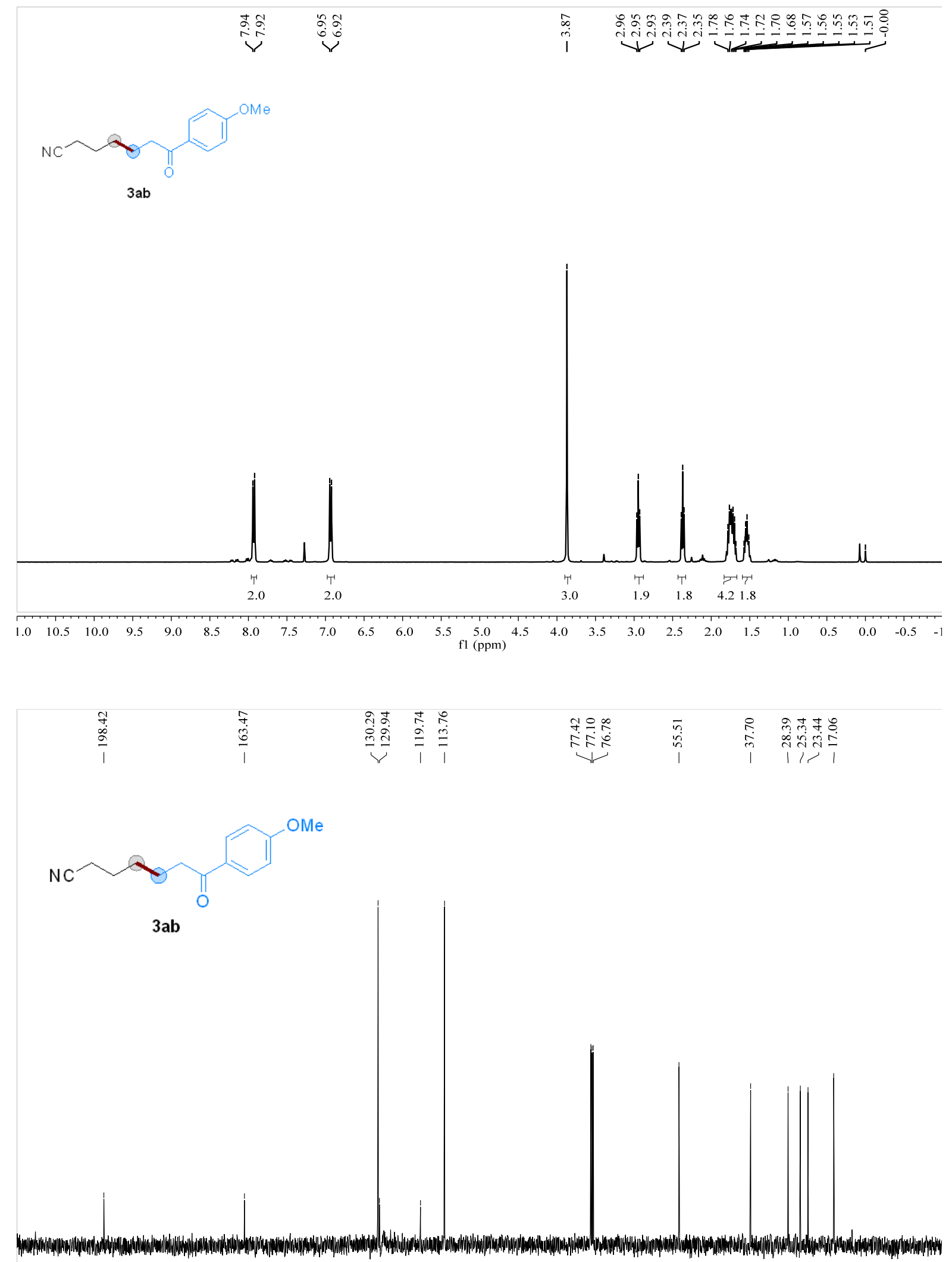

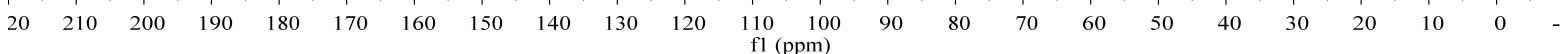


${ }^{1} \mathrm{H}$ NMR (400 MHz, $\left.\mathrm{CDCl}_{3}\right)$ and ${ }^{13} \mathrm{C}$ NMR (100 MHz, $\left.\mathrm{CDCl}_{3}\right)$ spectra of product $3 \mathrm{bb}$
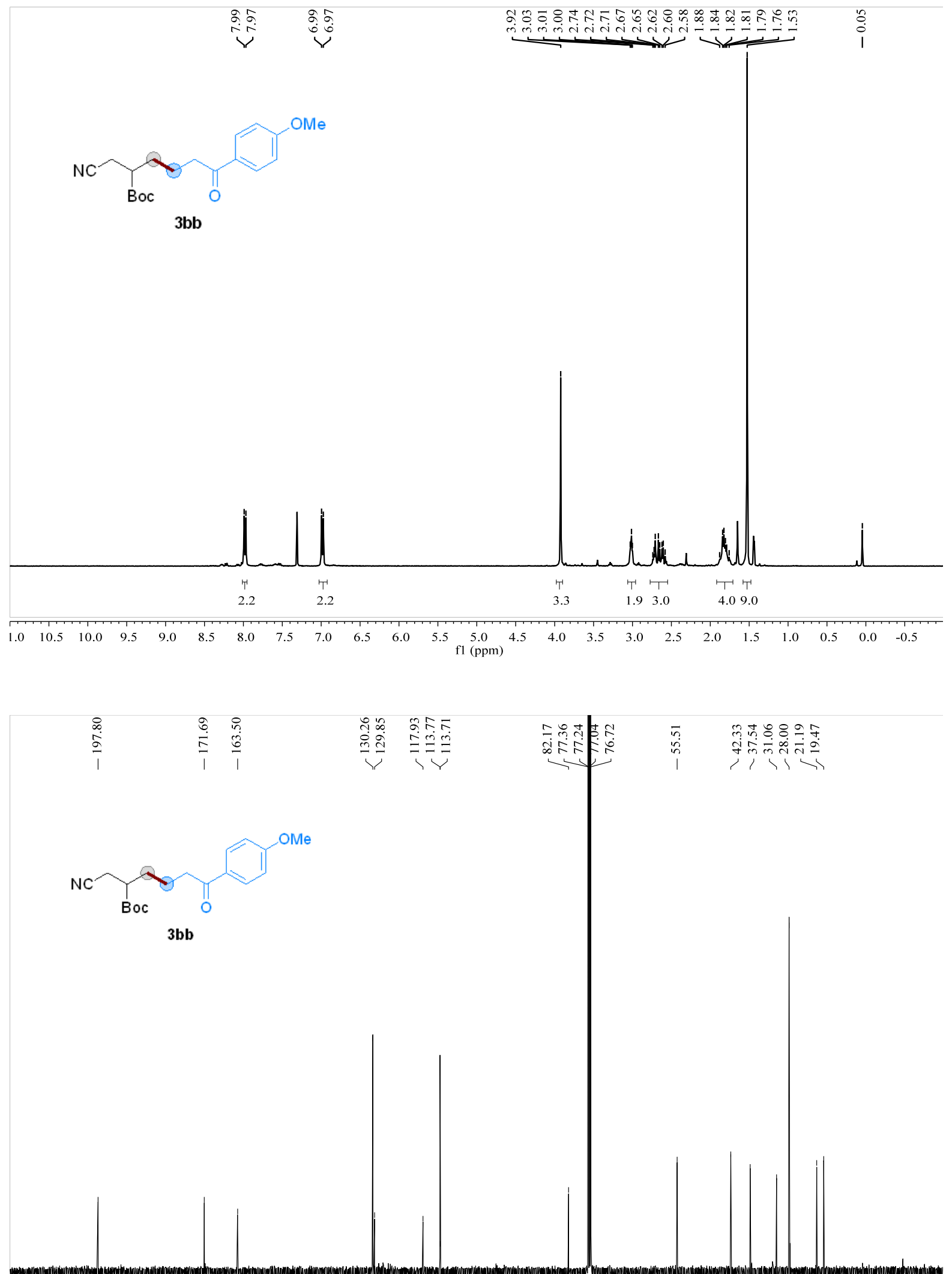

$\begin{array}{rlllllllllllllllllllll}210 & 200 & 190 & 180 & 170 & 160 & 150 & 140 & 130 & 120 & \begin{array}{c}110 \\ \mathrm{fl}(\mathrm{ppm})\end{array} & \begin{array}{l}100 \\ 80\end{array} & 70 & 60 & 50 & 40 & 30 & 20 & 10 & 0 & -\end{array}$ 
${ }^{1} \mathrm{H}$ NMR (400 MHz, $\left.\mathrm{CDCl}_{3}\right)$ and ${ }^{13} \mathrm{C}$ NMR (100 MHz, $\left.\mathrm{CDCl}_{3}\right)$ spectra of product 3cb
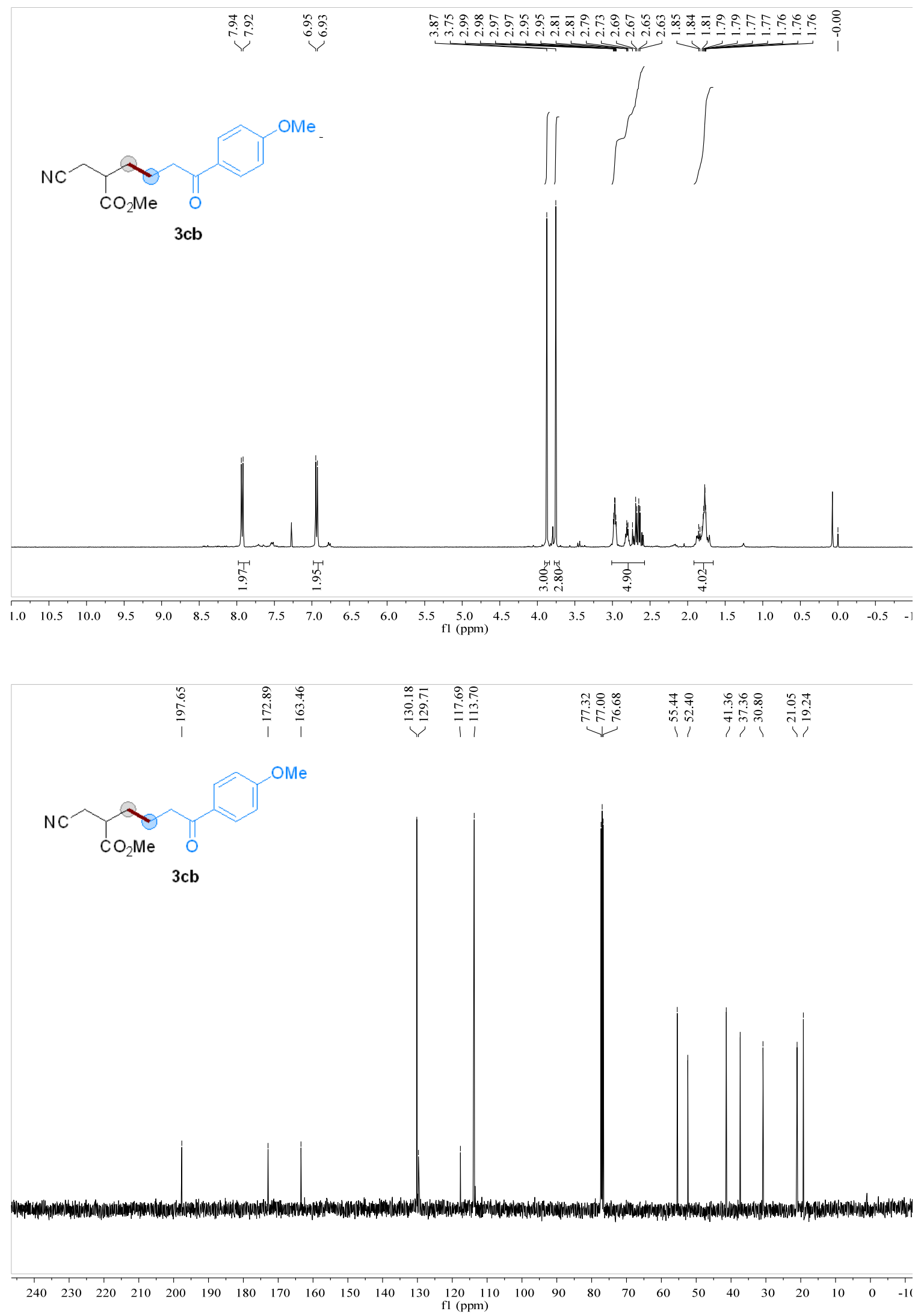
${ }^{1} \mathrm{H}$ NMR (400 MHz, $\left.\mathrm{CDCl}_{3}\right)$ and ${ }^{13} \mathrm{C}$ NMR (100 MHz, $\left.\mathrm{CDCl}_{3}\right)$ spectra of product 3db
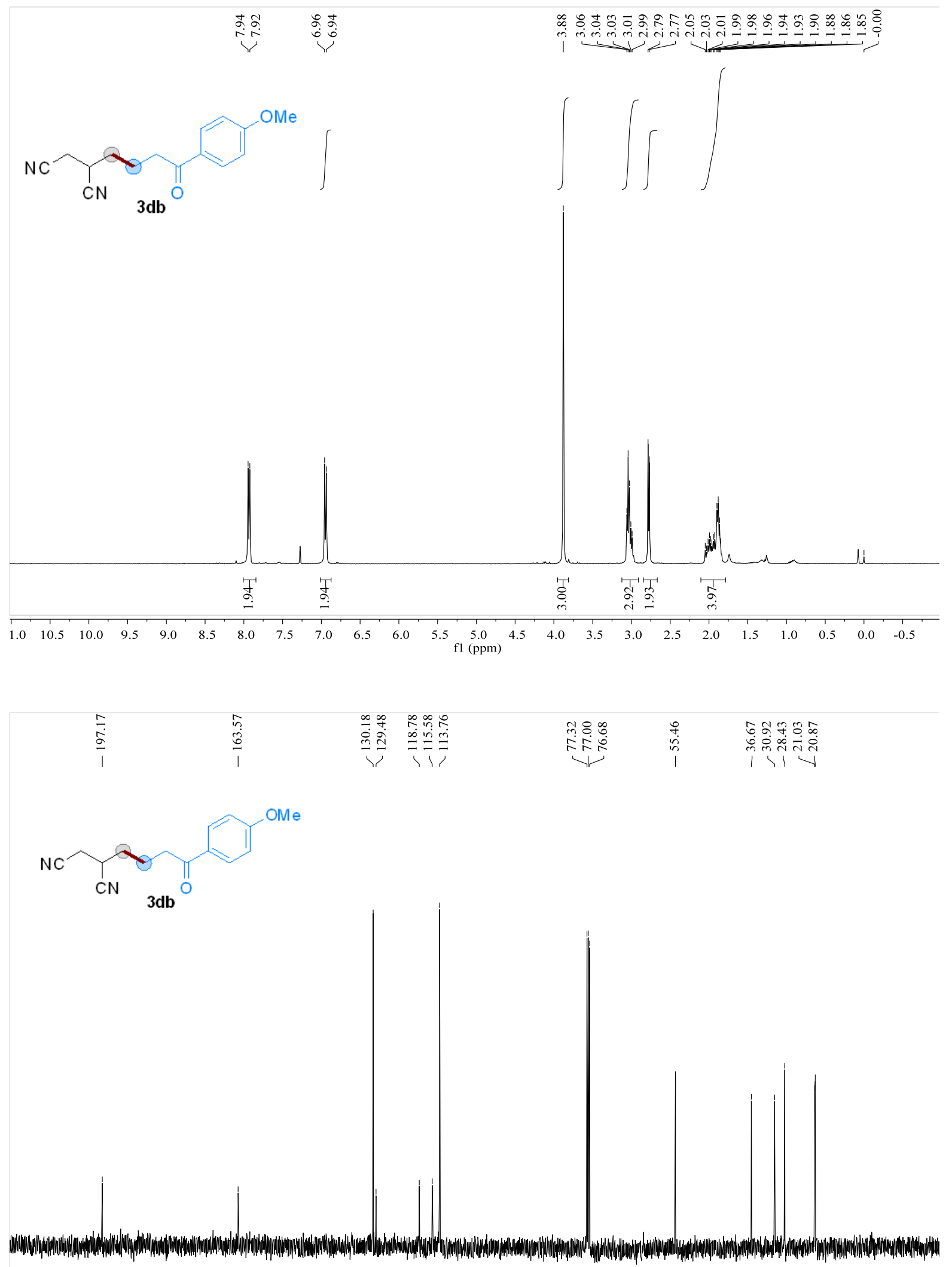

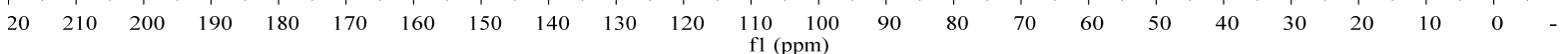


${ }^{1} \mathrm{H}$ NMR (400 MHz, $\left.\mathrm{CDCl}_{3}\right)$ and ${ }^{13} \mathrm{C}$ NMR (100 MHz, $\left.\mathrm{CDCl}_{3}\right)$ spectra of product 3eb
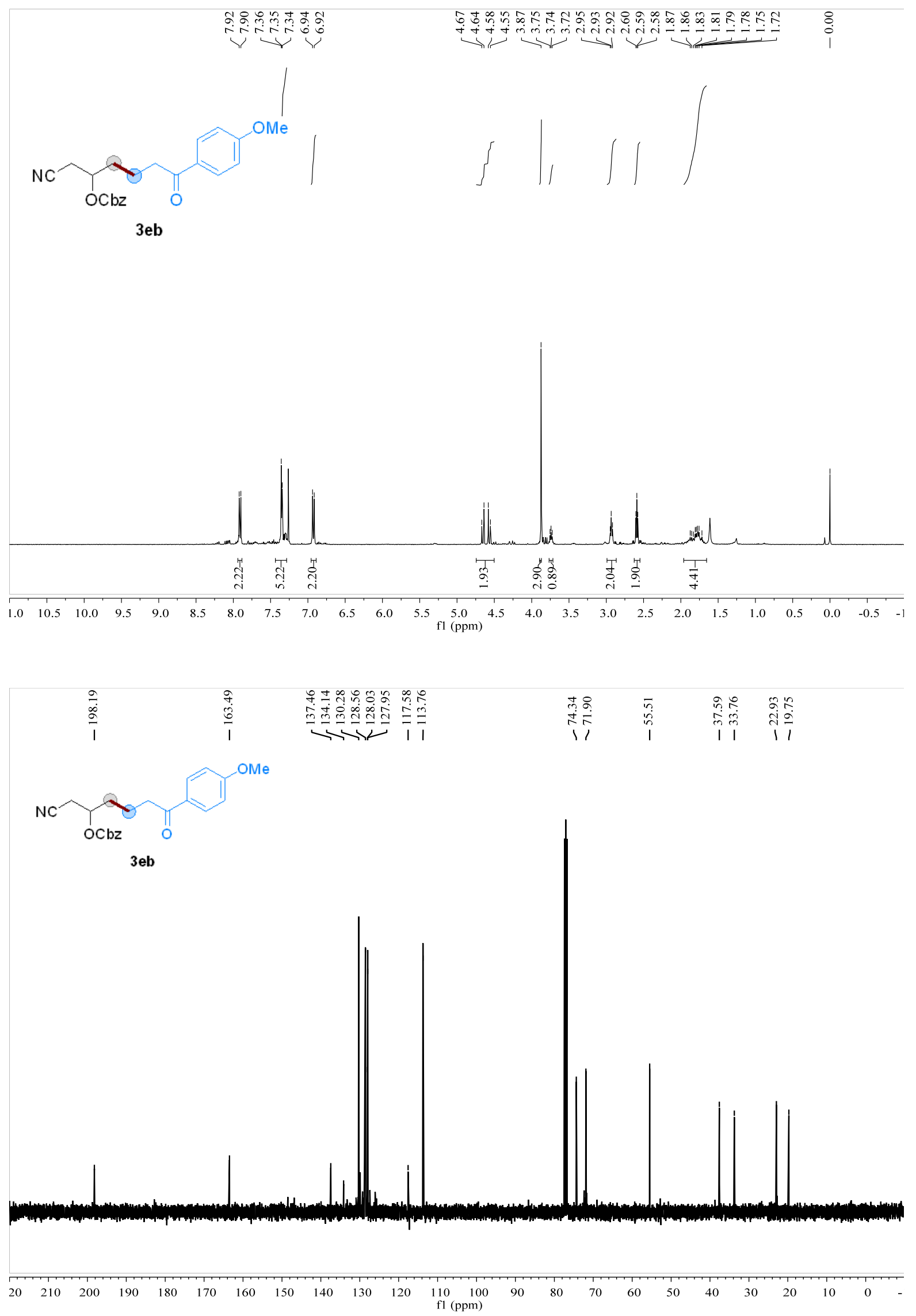
${ }^{1} \mathrm{H}$ NMR (400 MHz, $\left.\mathrm{CDCl}_{3}\right)$ and ${ }^{13} \mathrm{C}$ NMR (100 MHz, $\left.\mathrm{CDCl}_{3}\right)$ spectra of product 3fb

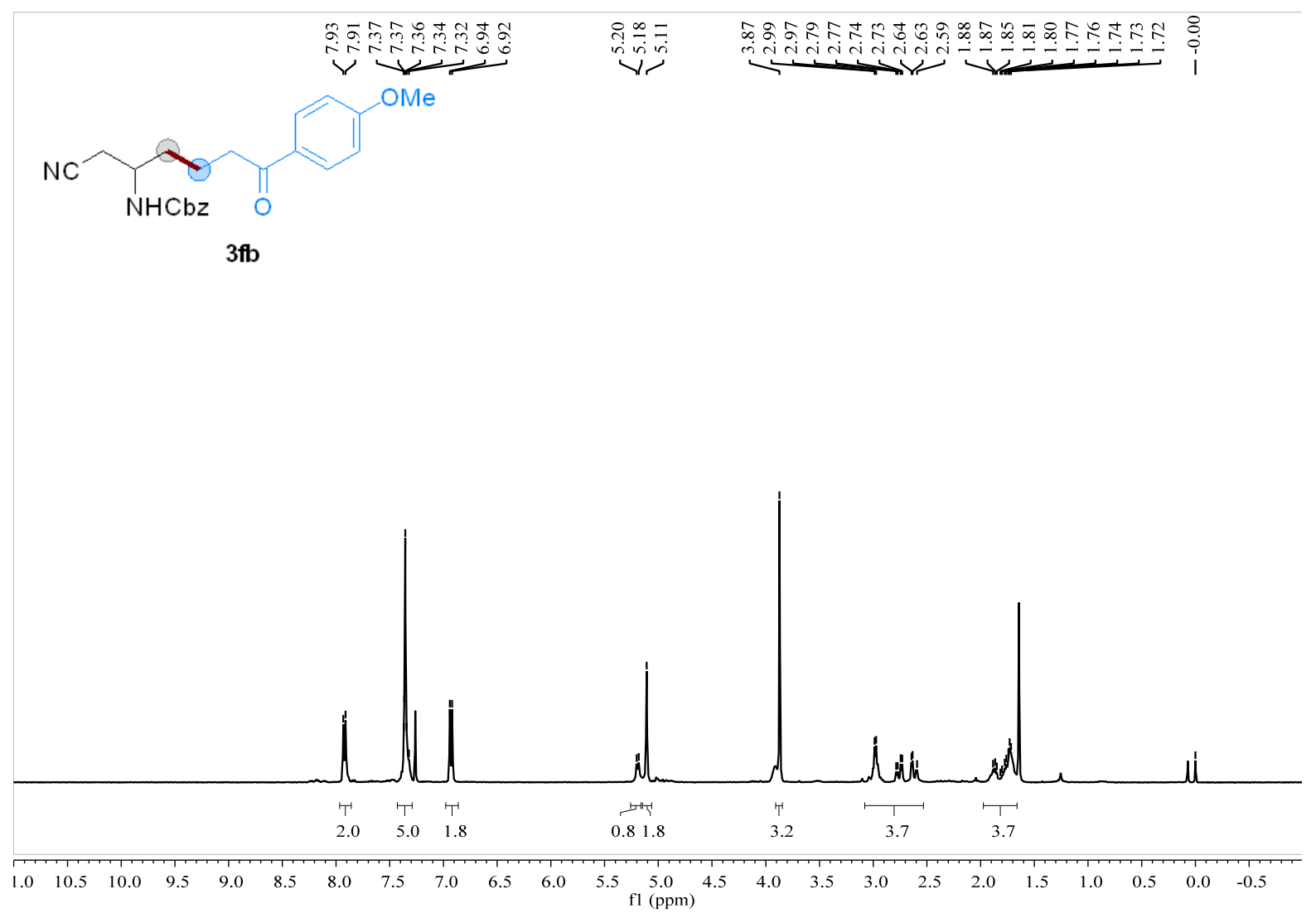

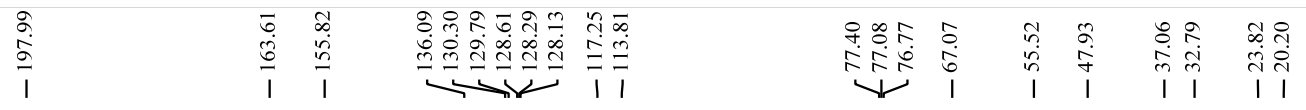

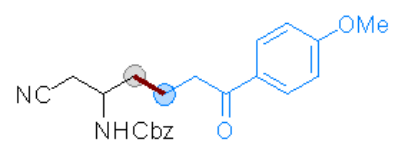

$3 \mathrm{fb}$

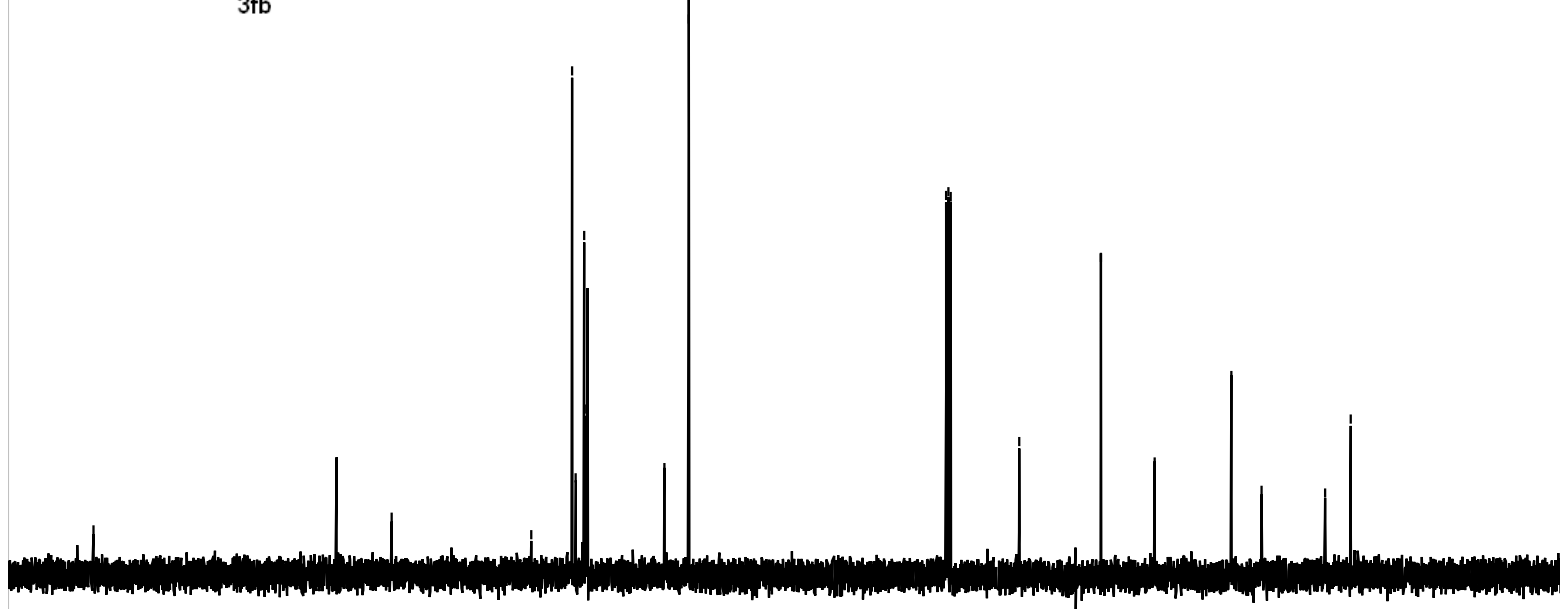

\begin{tabular}{lllllllllllllllllllllllllll}
\hline 10 & 200 & 190 & 180 & 170 & 160 & 150 & 140 & 130 & 120 & 110 & $\begin{array}{l}100 \\
\mathrm{fl}\end{array}$ & 90 & 80 & 70 & 60 & 50 & 40 & 30 & 20 & 10 & 0 & -
\end{tabular} 
${ }^{1} \mathrm{H}$ NMR (400 MHz, $\left.\mathrm{CDCl}_{3}\right)$ and ${ }^{13} \mathrm{C}$ NMR (100 MHz, CDCl$)$ spectra of product 3gb
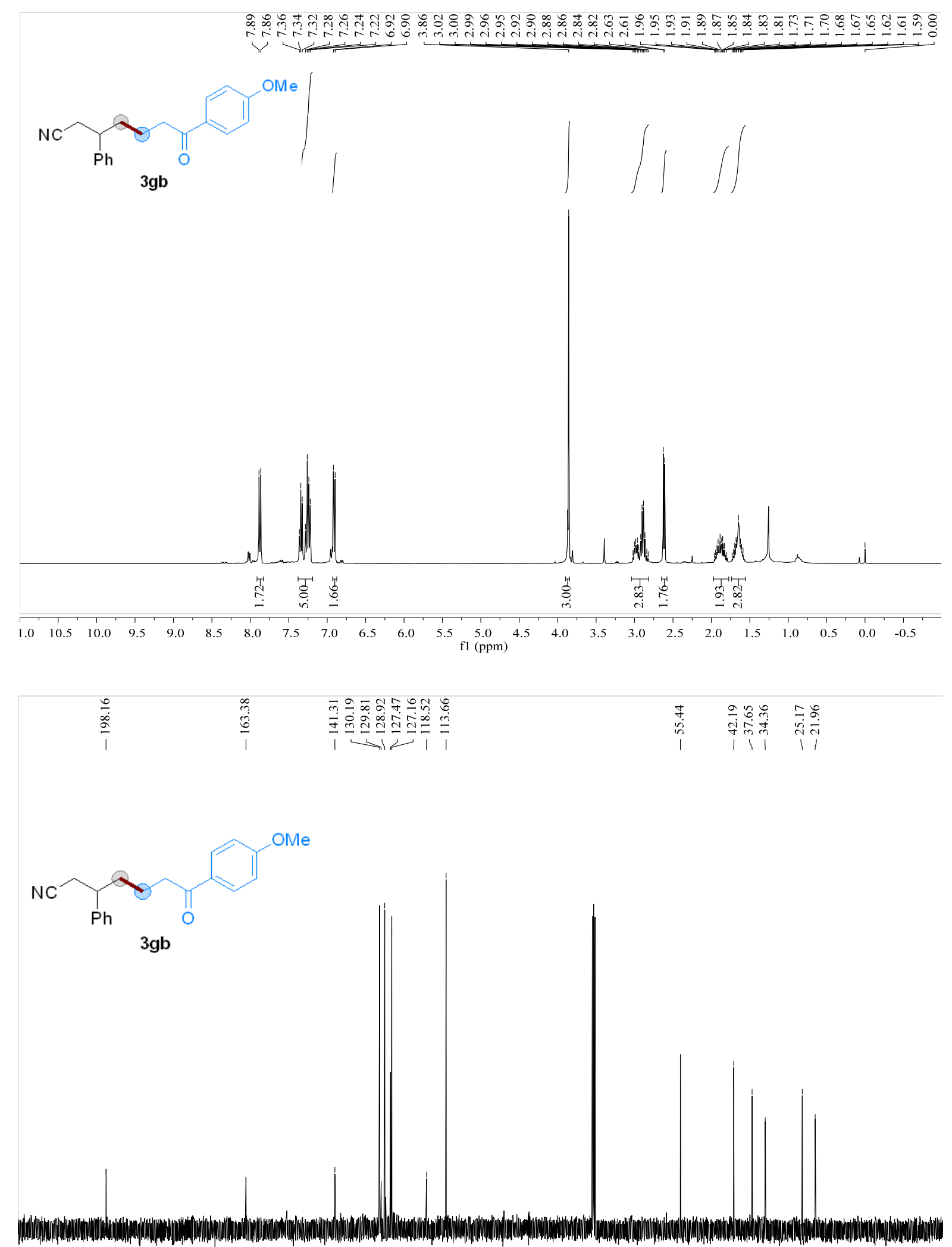

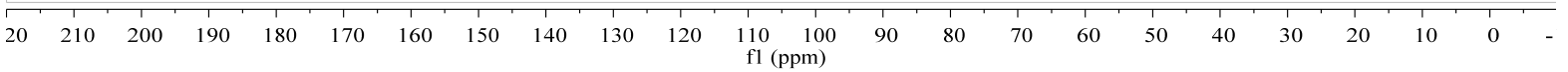


${ }^{1} \mathrm{H}$ NMR (400 MHz, CDCl $)$ and ${ }^{13} \mathrm{C}$ NMR (100 MHz, $\left.\mathrm{CDCl}_{3}\right)$ spectra of product $3 \mathrm{hb}$
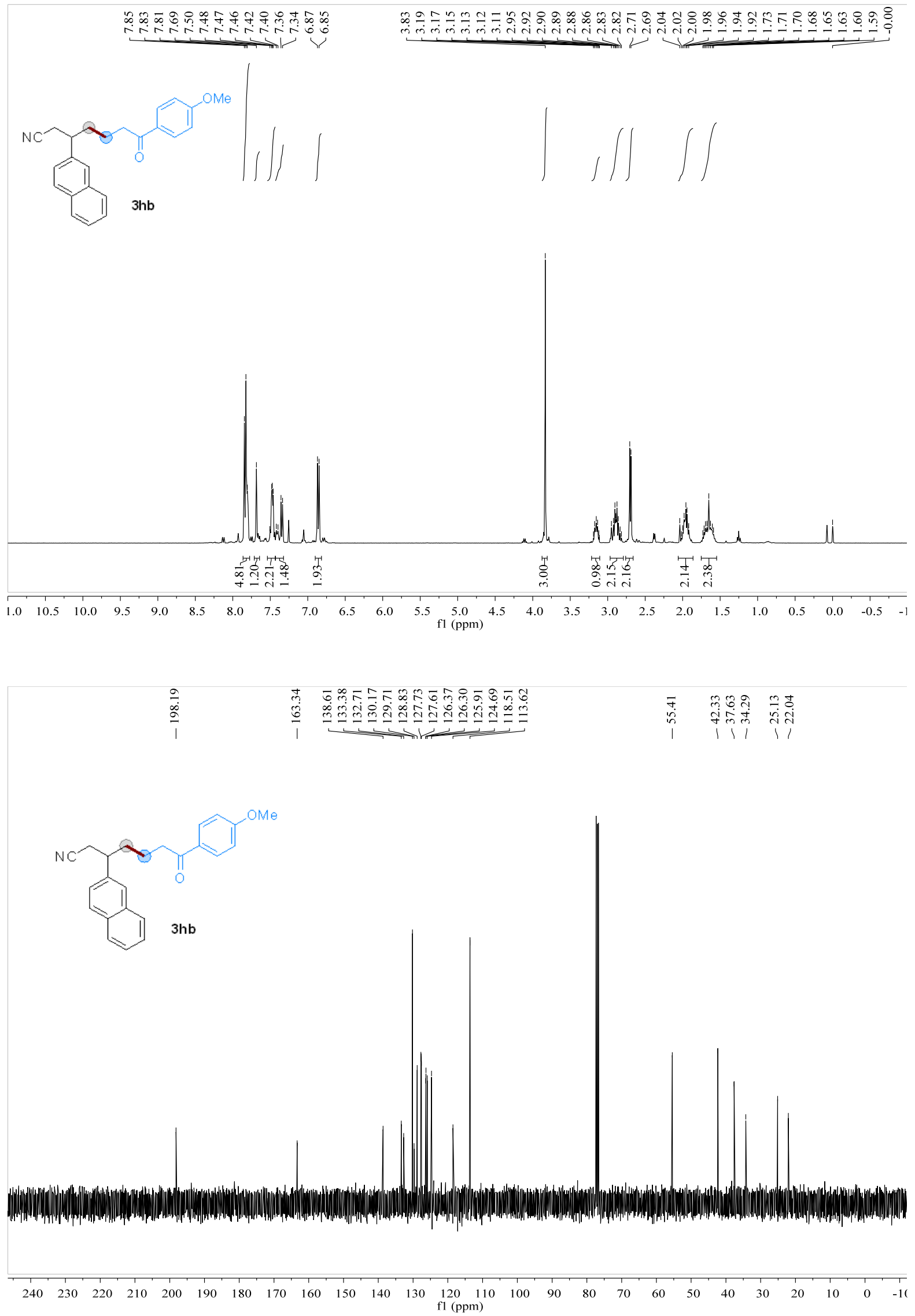
${ }^{1} \mathrm{H}$ NMR (400 MHz, $\left.\mathrm{CDCl}_{3}\right)$ and ${ }^{13} \mathrm{C}$ NMR (100 MHz, CDCl$)$ spectra of product 3ib
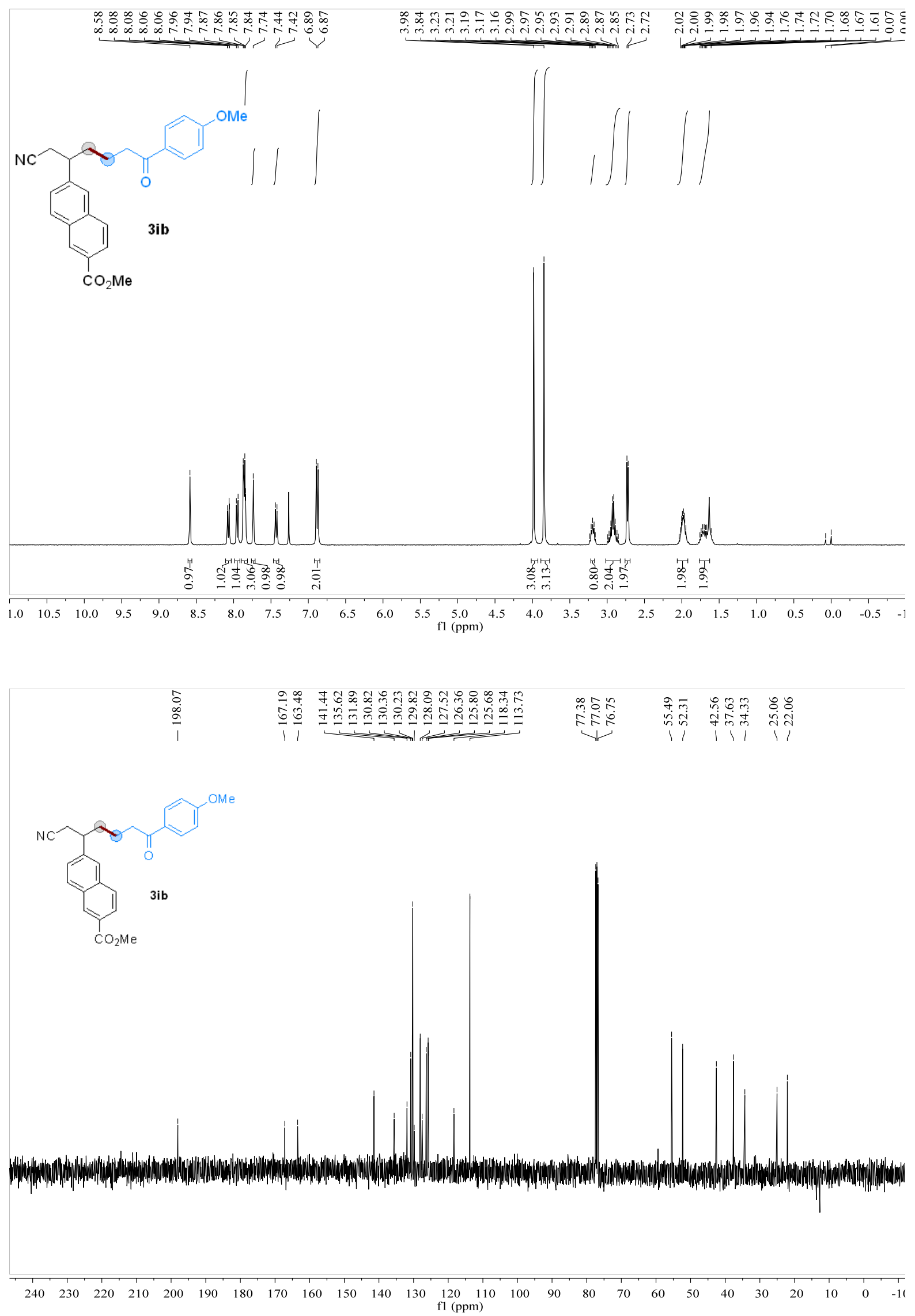
${ }^{1} \mathrm{H}$ NMR (400 MHz, CDCl 3$)$ and ${ }^{13} \mathrm{C}$ NMR (100 MHz, $\left.\mathrm{CDCl}_{3}\right)$ spectra of product 3jb
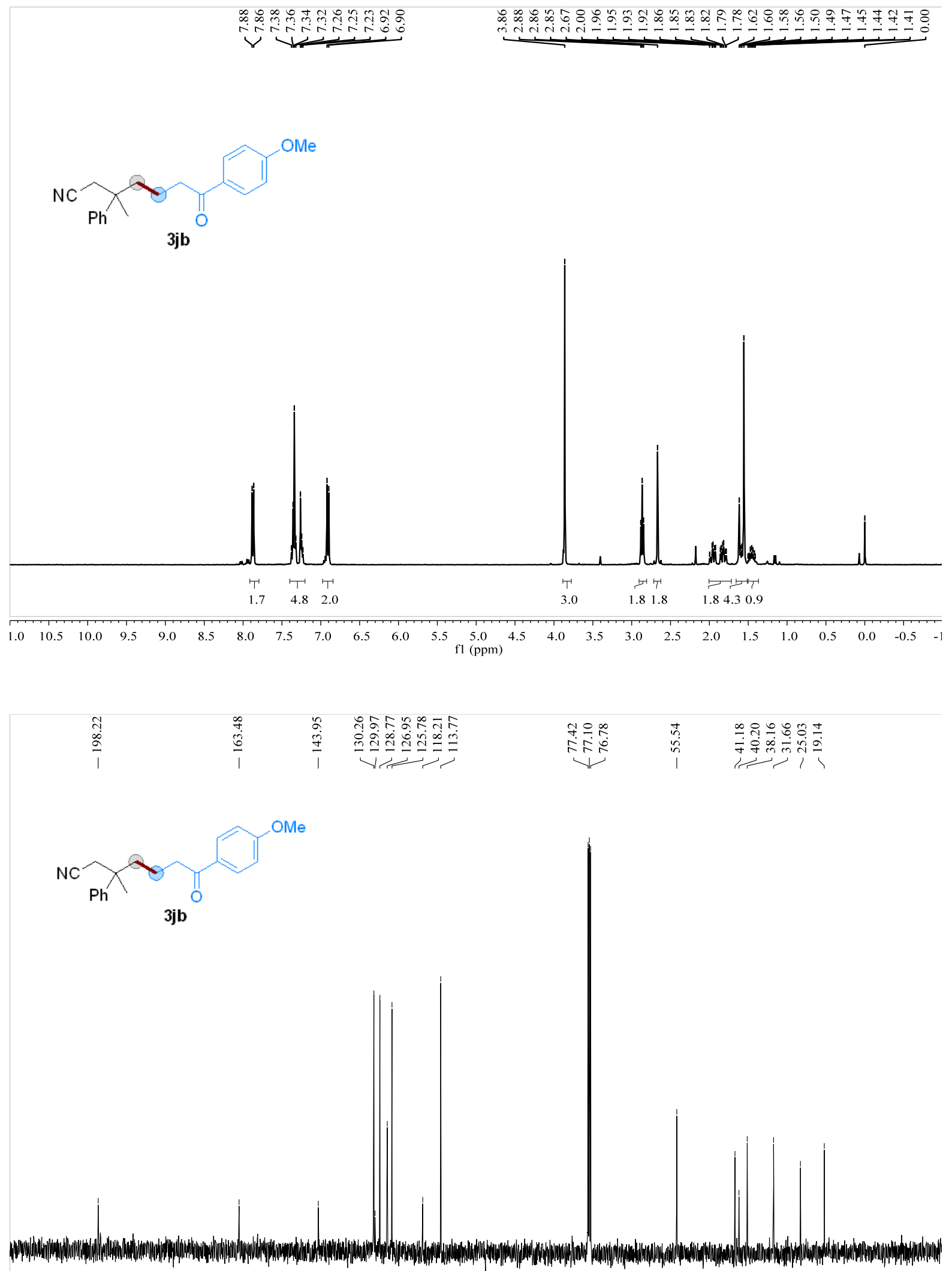

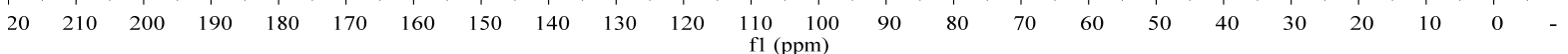


${ }^{1} \mathrm{H}$ NMR (400 MHz, $\left.\mathrm{CDCl}_{3}\right)$ and ${ }^{13} \mathrm{C}$ NMR (100 MHz, $\left.\mathrm{CDCl}_{3}\right)$ spectra of product 3kb
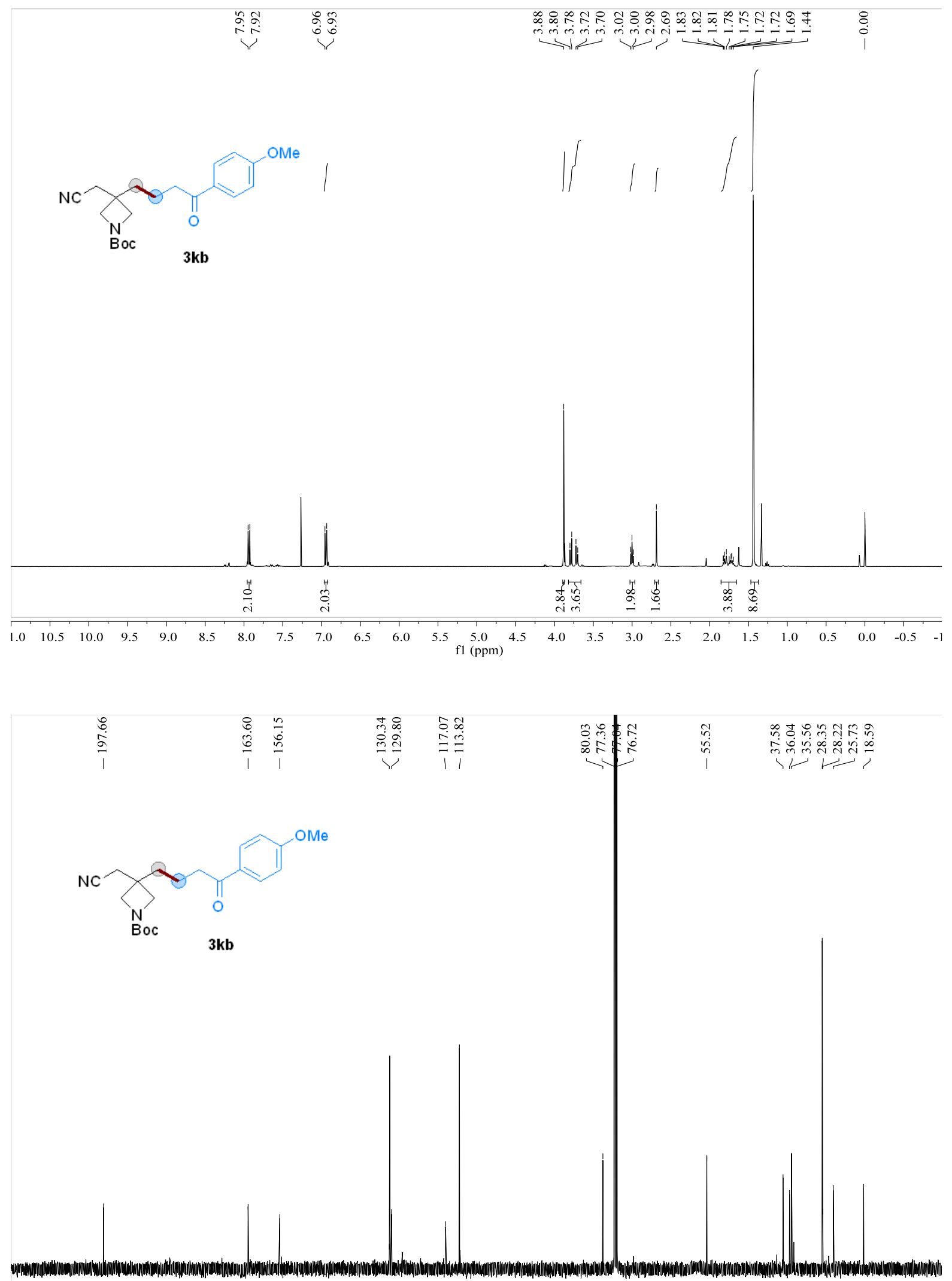

$\begin{array}{lllllllllllllllllllll}210 & 200 & 190 & 180 & 170 & 160 & 150 & 140 & 130 & 120 & 110 & 100 & 90 & 80 & 70 & 60 & 50 & 40 & 30 & 20 & 10\end{array}$ 
${ }^{1} \mathrm{H}$ NMR (400 MHz, CDCl 3$)$ and ${ }^{13} \mathrm{C}$ NMR (100 MHz, CDCl3) spectra of product 3lb
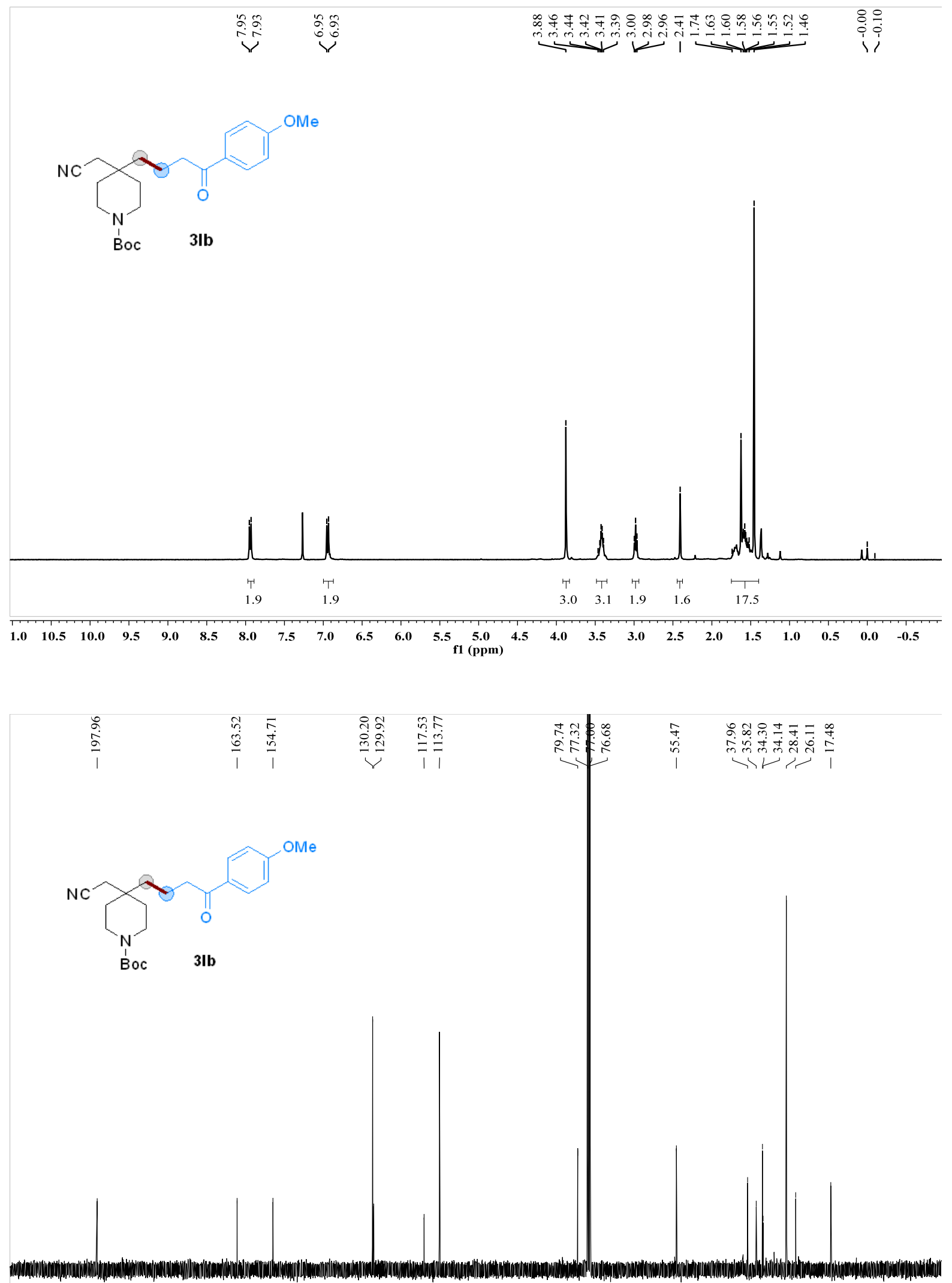

$\begin{array}{lllllllllllllllllllllllllllll}210 & 200 & 190 & 180 & 170 & 160 & 150 & 140 & 130 & 120 & 110 & 100 & 90 & 80 & 70 & 60 & 50 & 40 & 30 & 20 & 10 & 0 & -\end{array}$ 
${ }^{1} \mathrm{H}$ NMR (400 MHz, CDCl$)$ and ${ }^{13} \mathrm{C}$ NMR (100 MHz, $\left.\mathrm{CDCl}_{3}\right)$ spectra of product 3mb
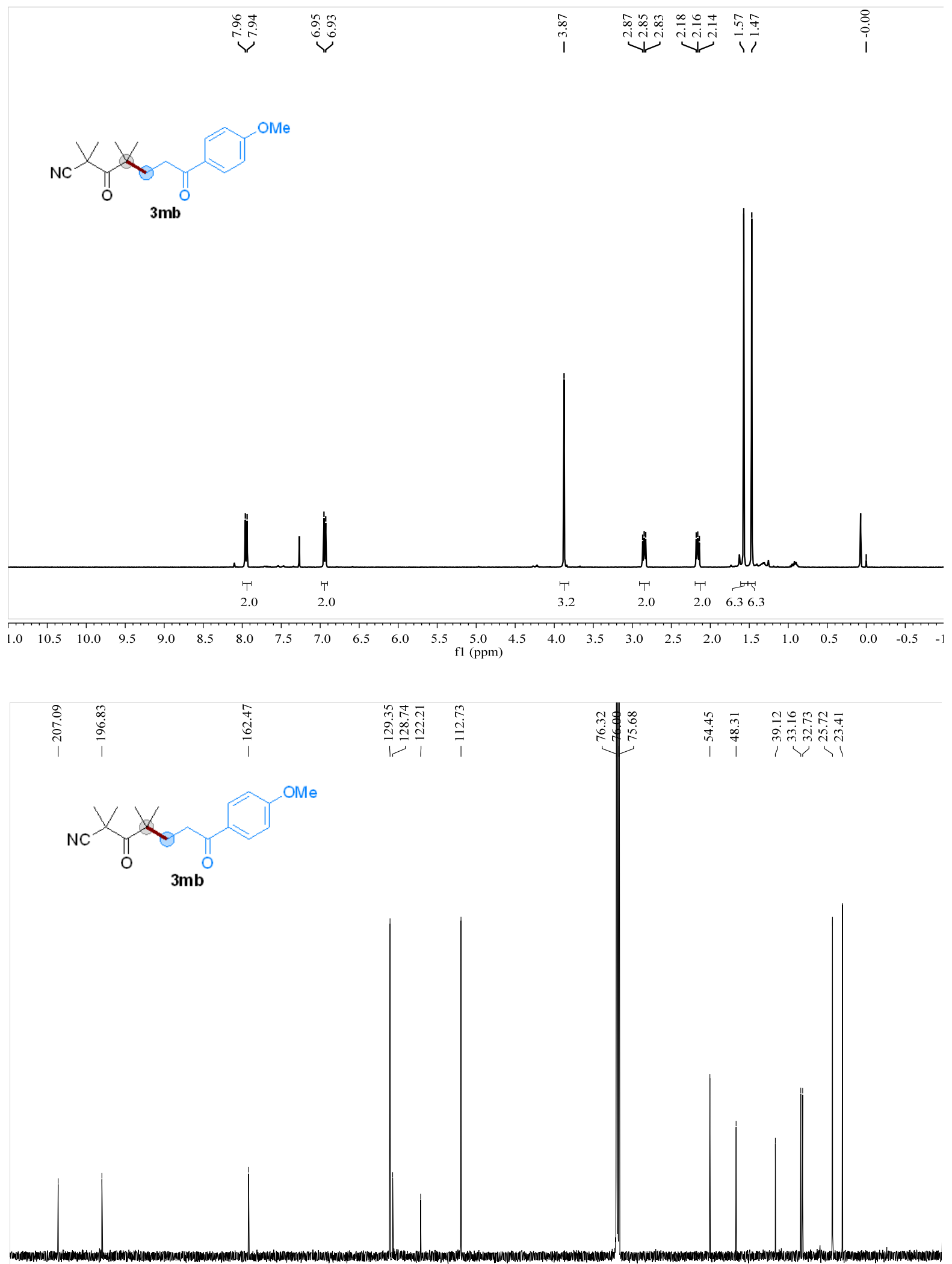

$\begin{array}{llllllllllllllllllllllll}210 & 200 & 190 & 180 & 170 & 160 & 150 & 140 & 130 & 120 & 110 & 100 & 90 & 80 & 70 & 60 & 50 & 40 & 30 & 20 & 10\end{array}$ 
${ }^{1} \mathrm{H}$ NMR (400 MHz, $\left.\mathrm{CDCl}_{3}\right)$ and ${ }^{13} \mathrm{C}$ NMR (100 MHz, $\left.\mathrm{CDCl}_{3}\right)$ spectra of product 3nb
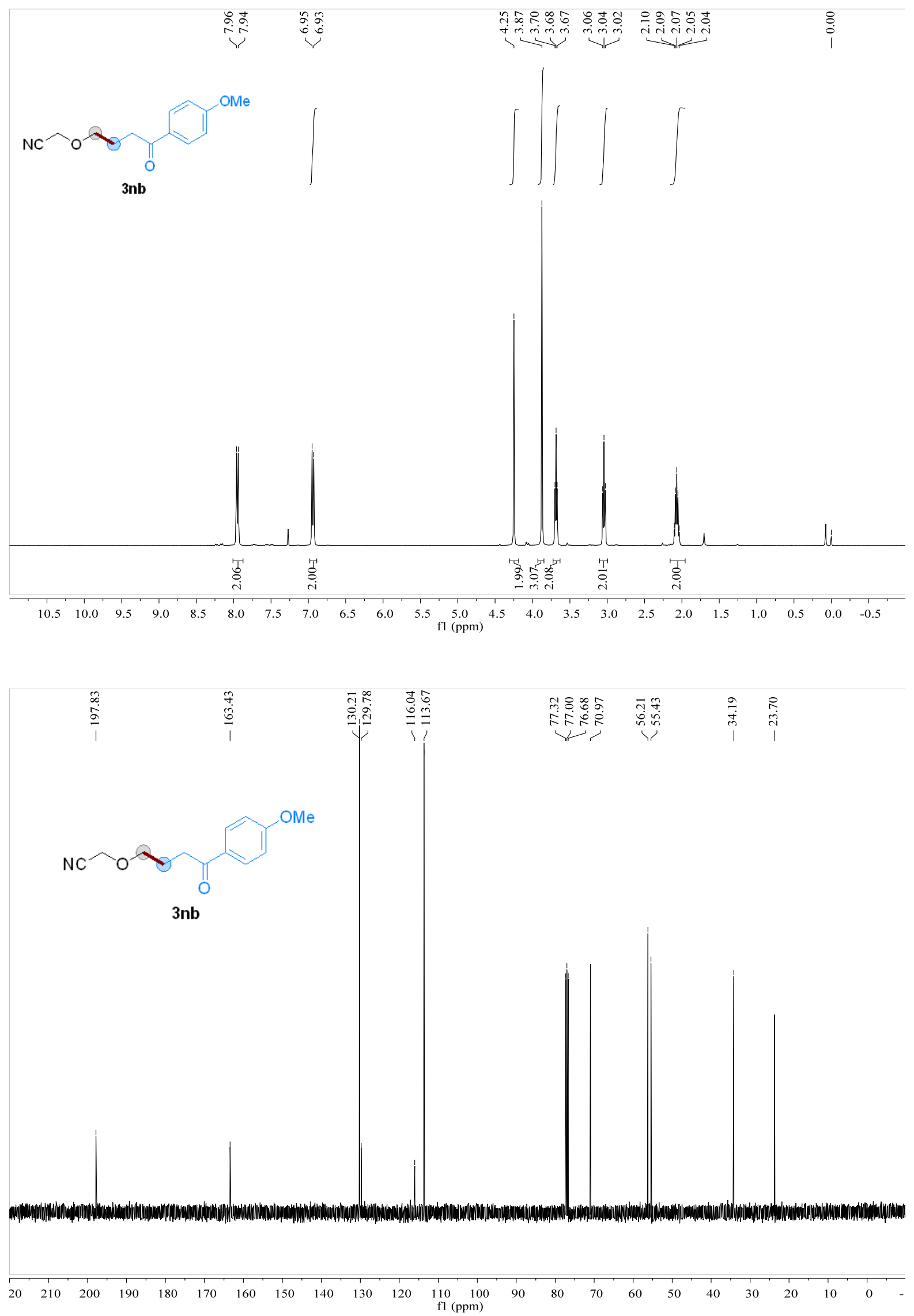
${ }^{1} \mathrm{H}$ NMR (400 MHz, $\left.\mathrm{CDCl}_{3}\right)$ and ${ }^{13} \mathrm{C}$ NMR (100 MHz, $\left.\mathrm{CDCl}_{3}\right)$ spectra of product 3ob
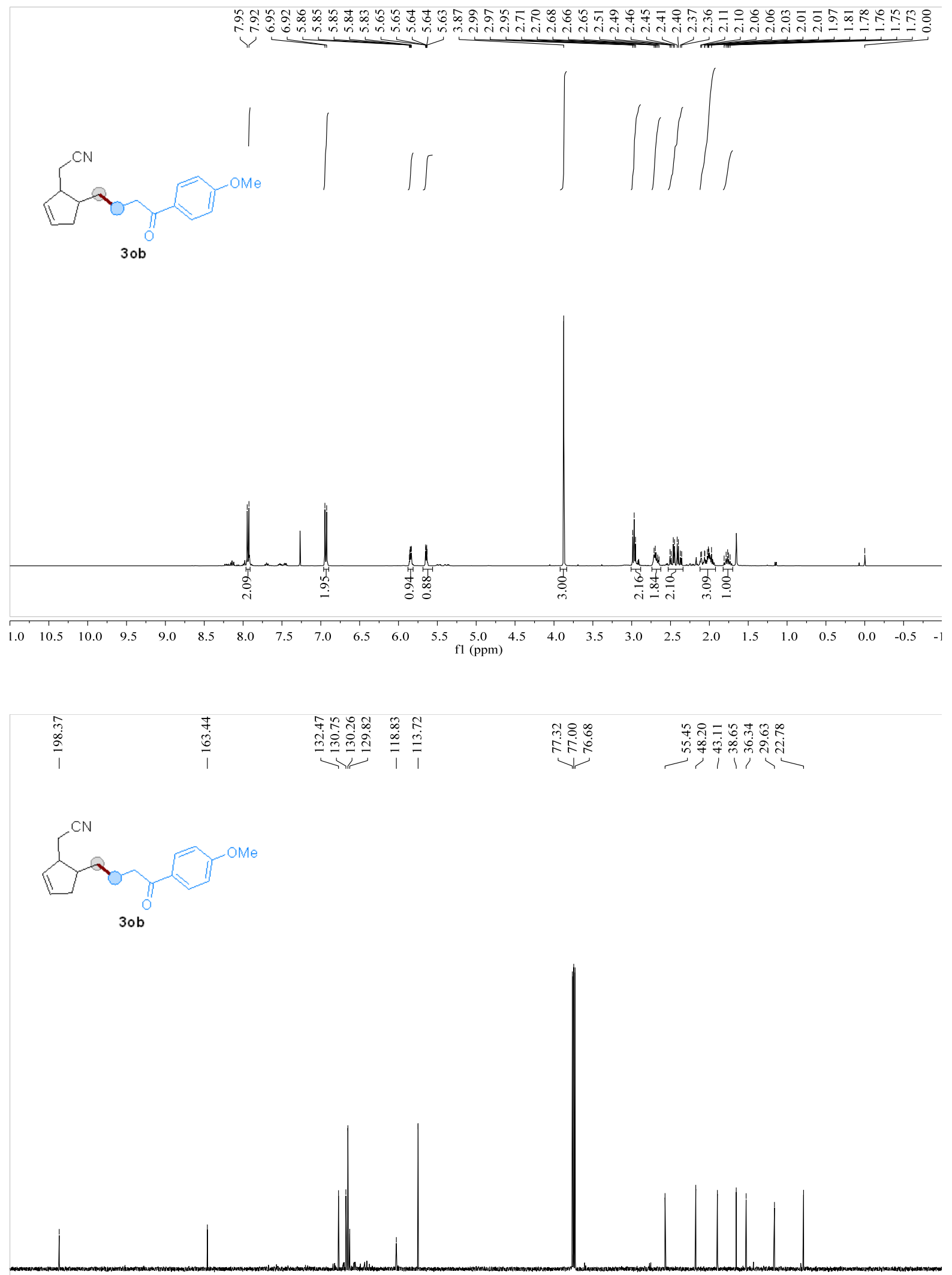

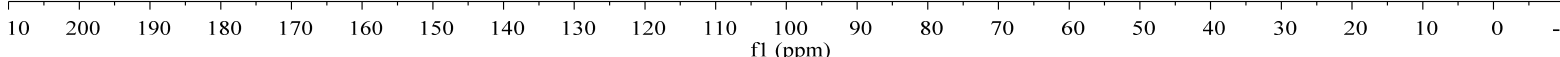


${ }^{1} \mathrm{H}$ NMR (400 MHz, CDCl $)$ and ${ }^{13} \mathrm{C}$ NMR (100 MHz, $\left.\mathrm{CDCl}_{3}\right)$ spectra of product 3pb
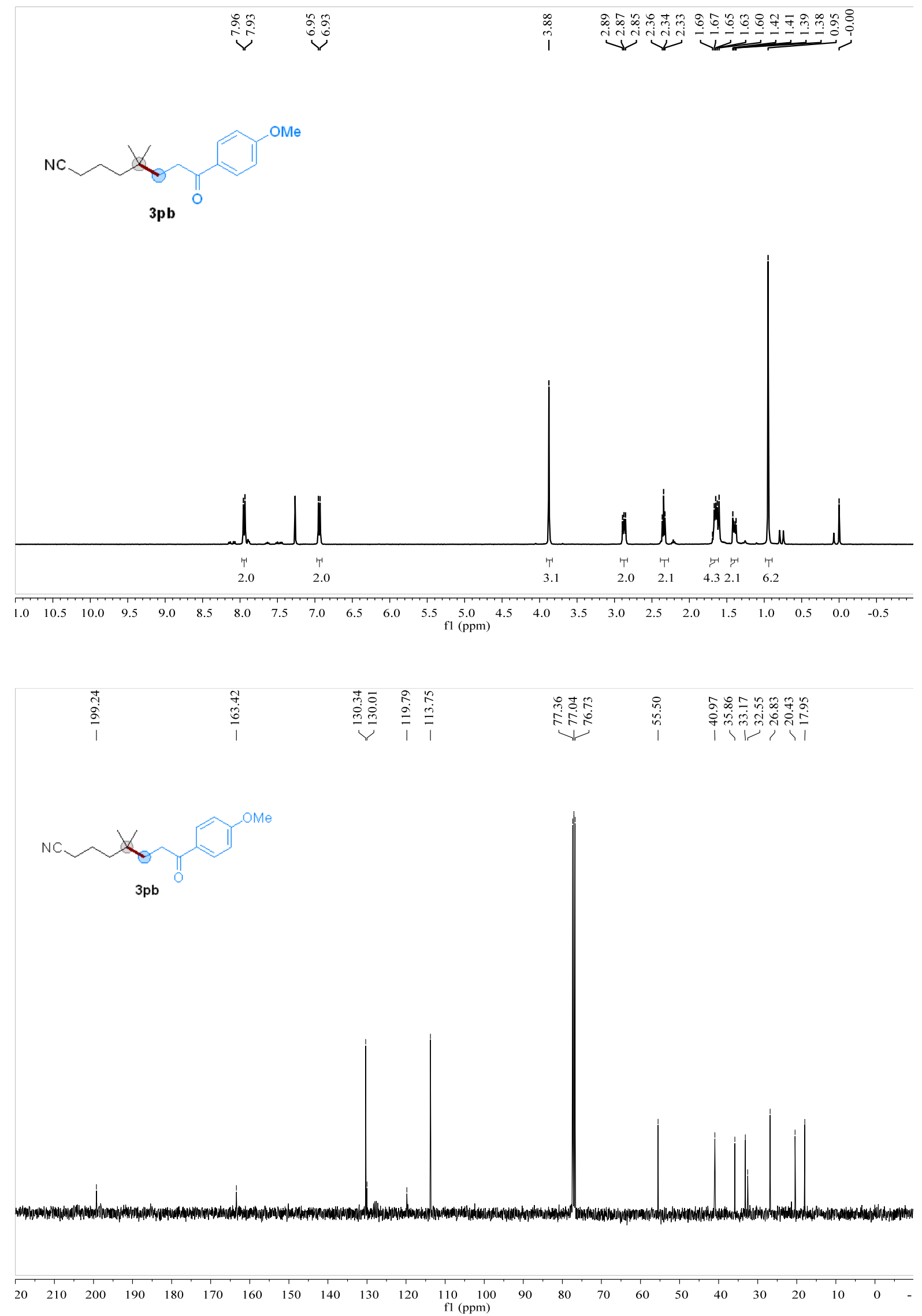
${ }^{1} \mathrm{H}$ NMR (400 MHz, $\left.\mathrm{CDCl}_{3}\right)$ and ${ }^{13} \mathrm{C}$ NMR (100 MHz, CDCl$)$ spectra of product 3qb
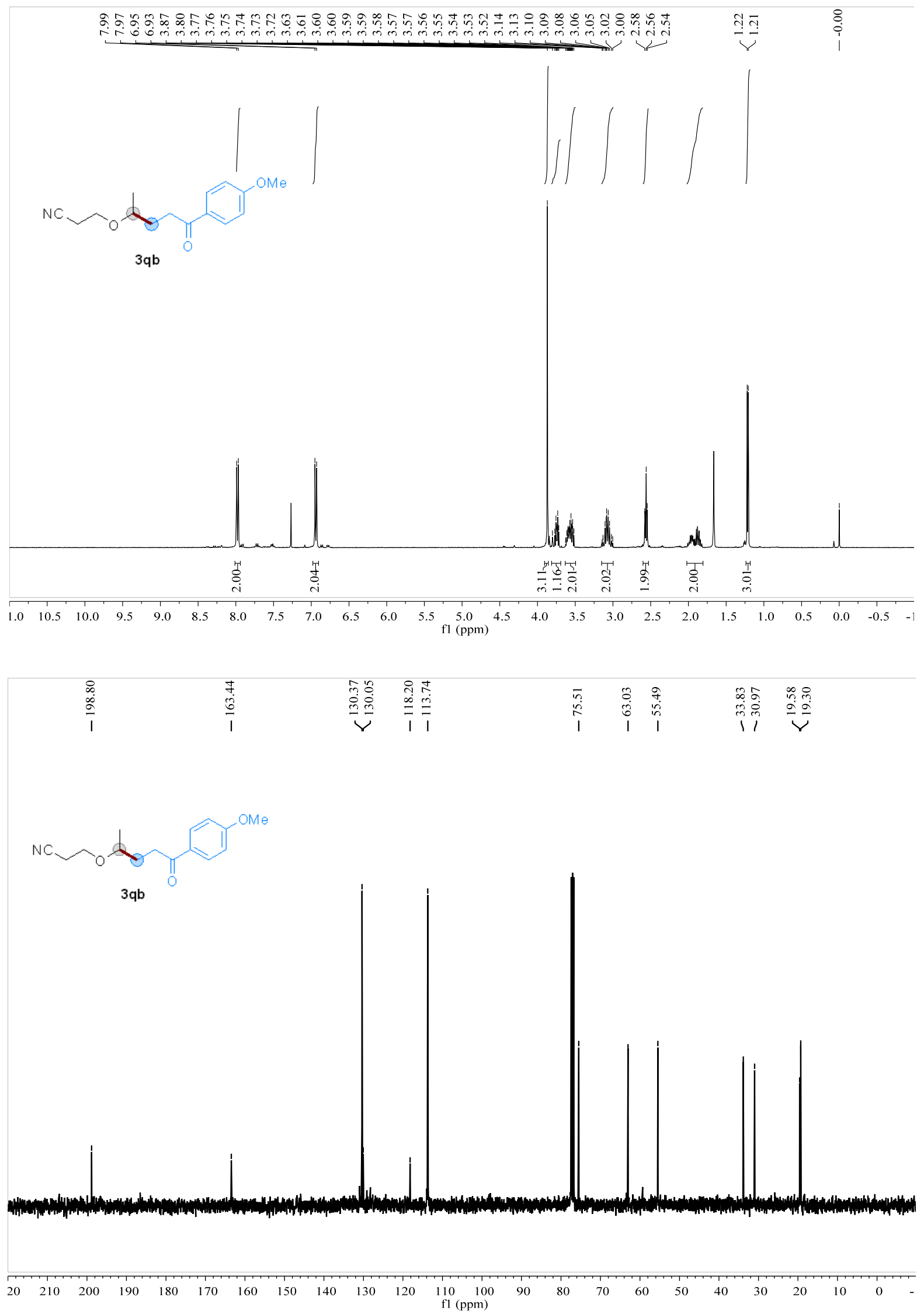
${ }^{1} \mathrm{H}$ NMR (400 MHz, $\left.\mathrm{CDCl}_{3}\right)$ and ${ }^{13} \mathrm{C}$ NMR (100 MHz, CDCl$)$ spectra of product 3aa

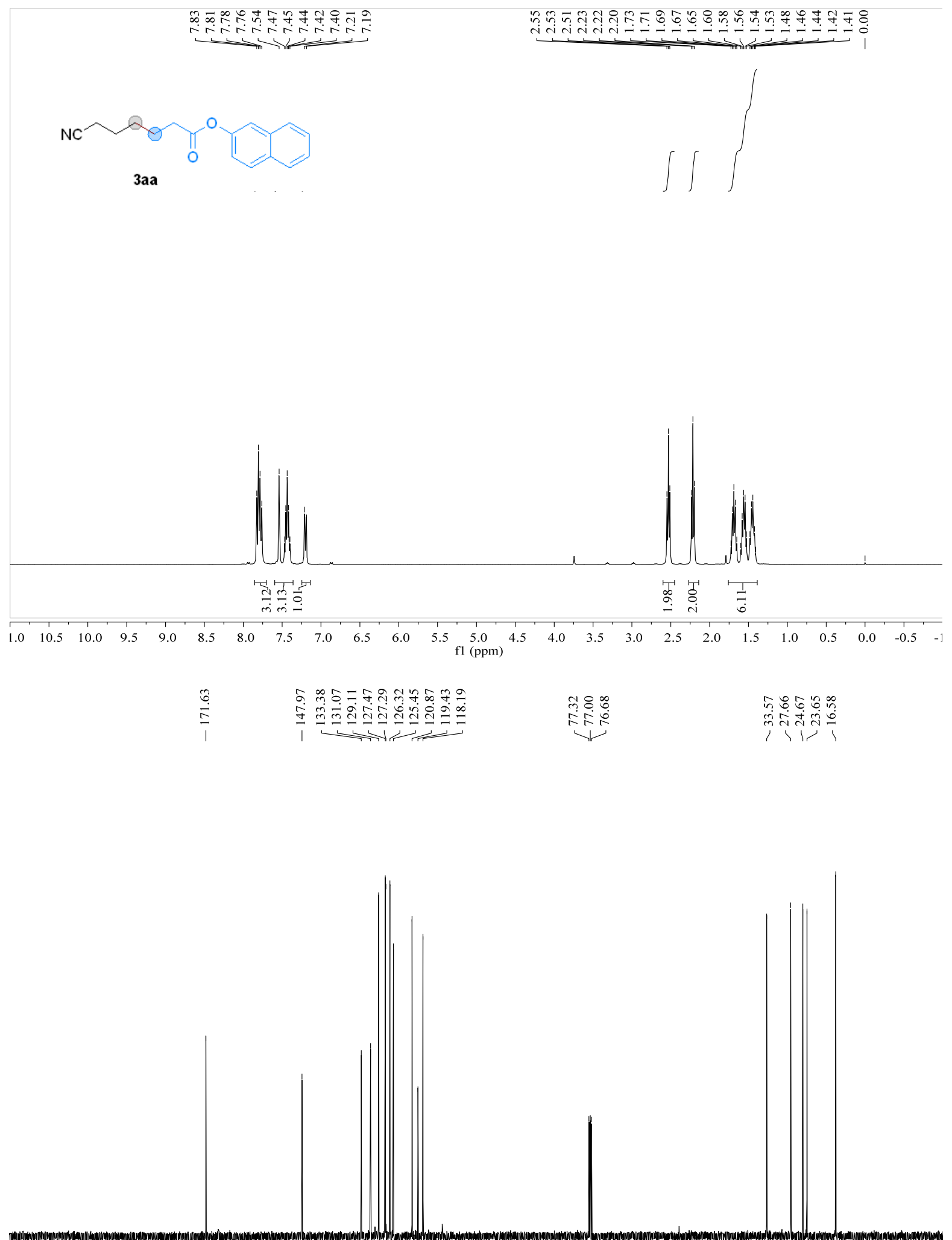

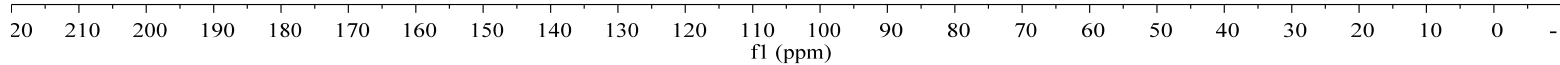


${ }^{1} \mathrm{H}$ NMR (400 MHz, $\left.\mathrm{CDCl}_{3}\right)$ and ${ }^{13} \mathrm{C}$ NMR (100 MHz, $\left.\mathrm{CDCl}_{3}\right)$ spectra of product 3hc
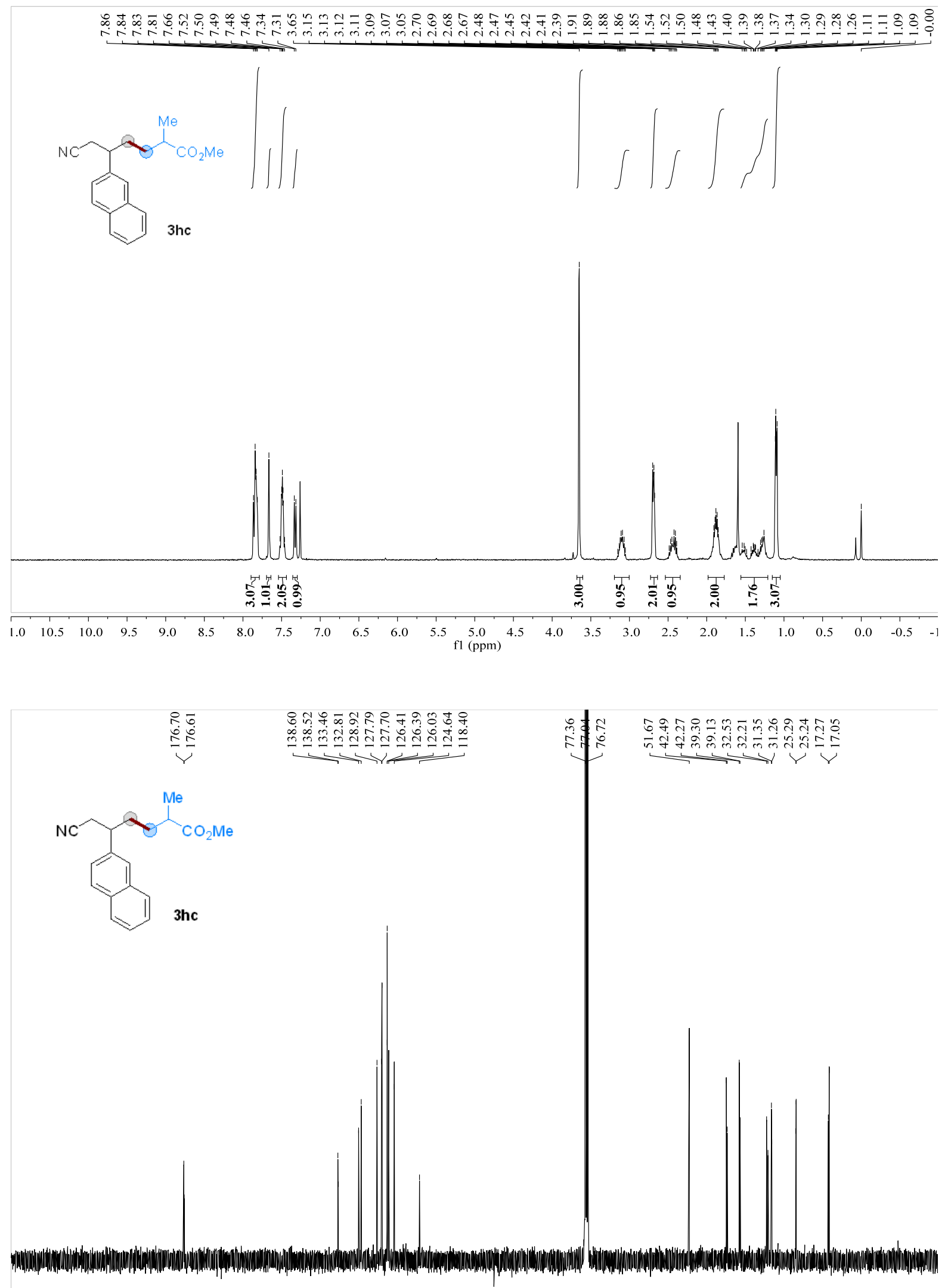

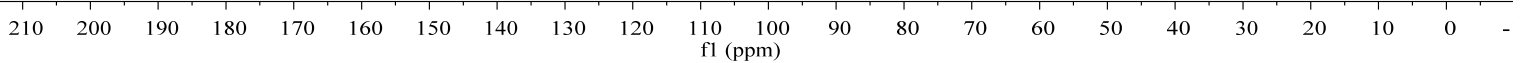


${ }^{1} \mathrm{H}$ NMR (400 MHz, $\left.\mathrm{CDCl}_{3}\right)$ and ${ }^{13} \mathrm{C}$ NMR (100 MHz, $\left.\mathrm{CDCl}_{3}\right)$ spectra of product 3hd
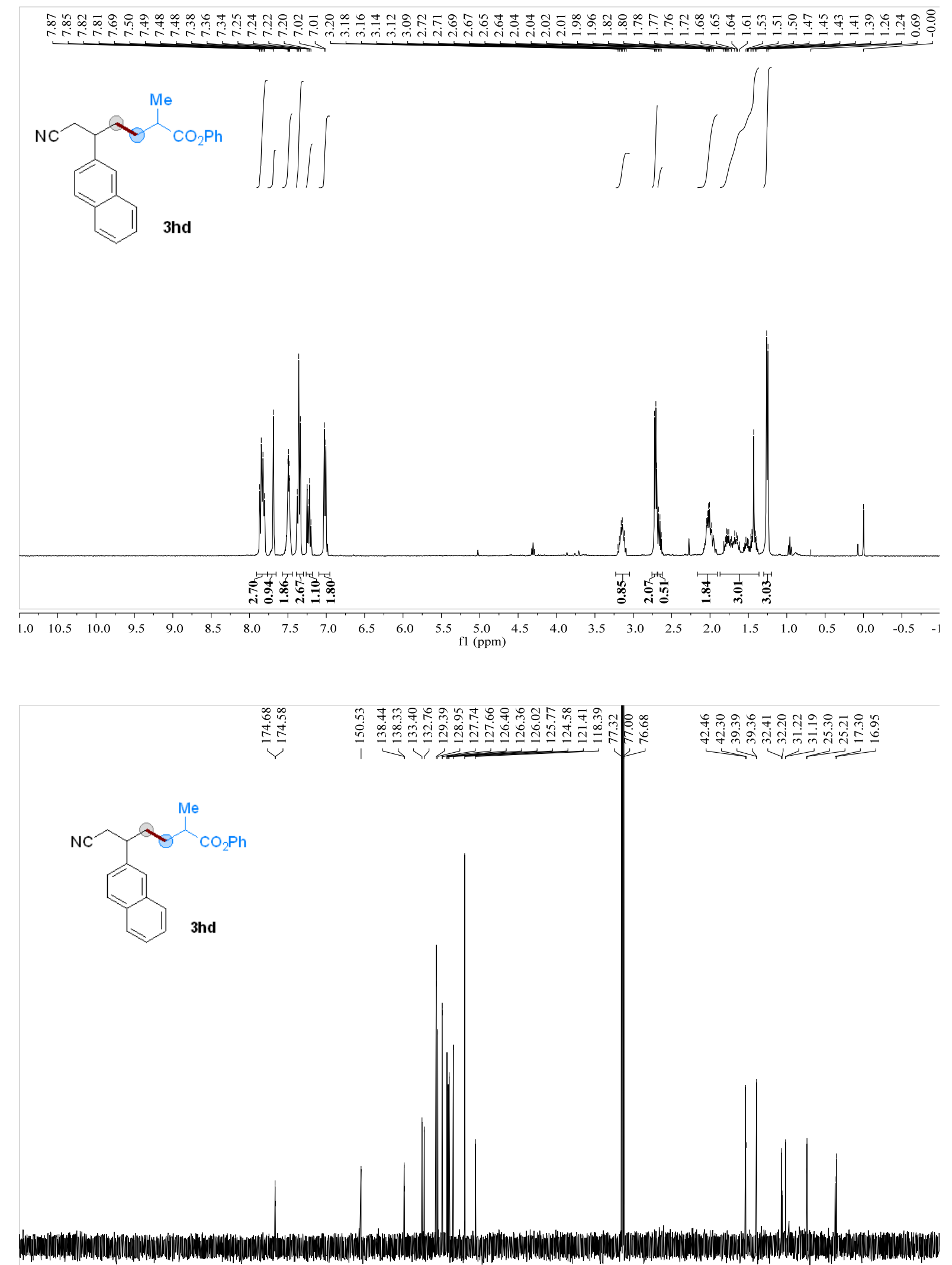

$\begin{array}{lllllllllllllllllllllllllllllllllllllllll}240 & 230 & 220 & 210 & 200 & 190 & 180 & 170 & 160 & 150 & 140 & 130 & 120 & 110 & 100 & 90 & 80 & 70 & 60 & 50 & 40 & 30 & 20 & 10 & 0 & -1(\end{array}$ 
${ }^{1} \mathrm{H}$ NMR (400 MHz, $\left.\mathrm{CDCl}_{3}\right)$ and ${ }^{13} \mathrm{C}$ NMR (100 MHz, $\left.\mathrm{CDCl}_{3}\right)$ spectra of product 3he
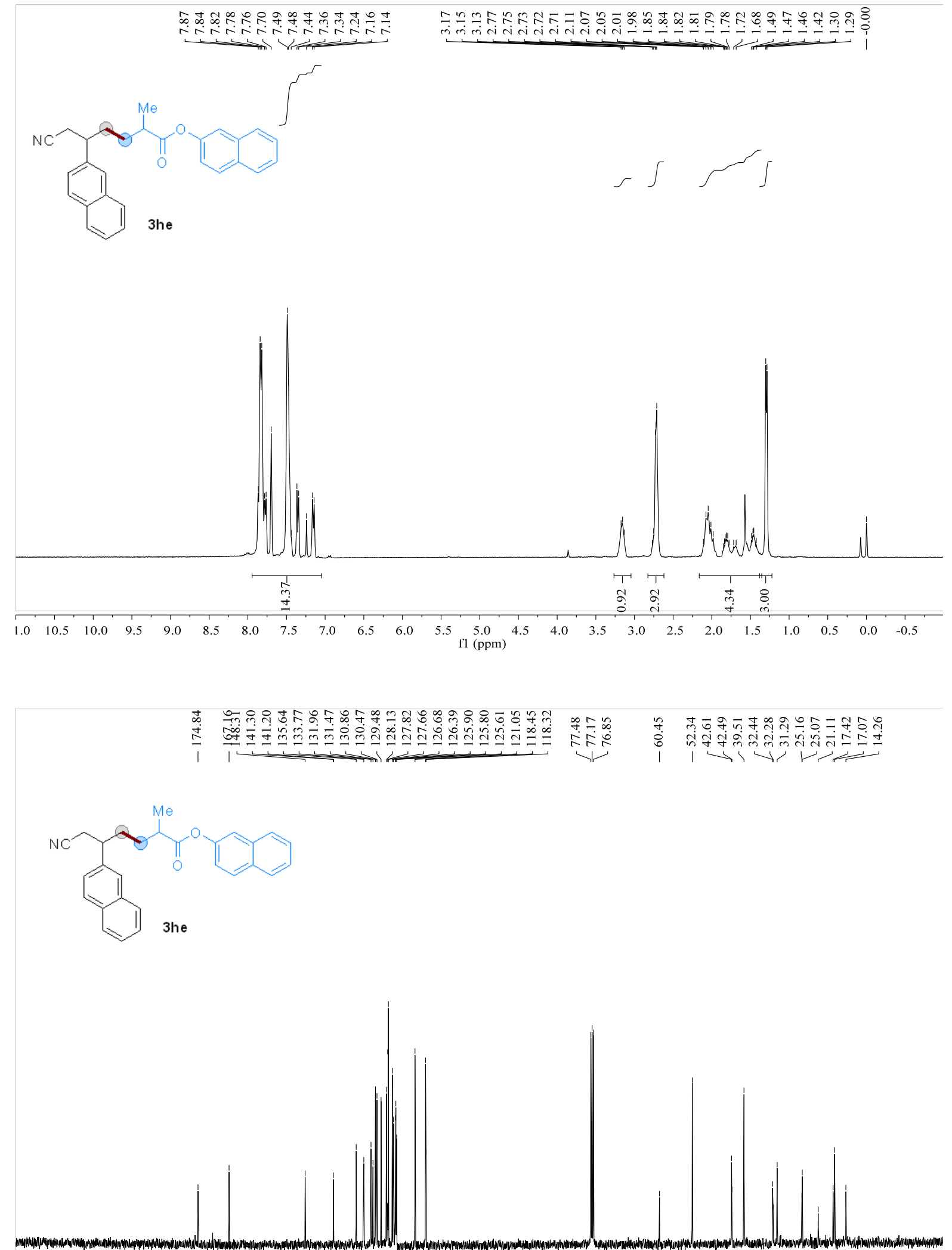

$\begin{array}{rlllllllllll}20 & 210 & 200 & 190 & 180 & 170 & 160 & 150 & 140 & 130 & 120 & \begin{array}{c}110 \\ \text { f1 (ppm) }\end{array}\end{array}$ 
${ }^{1} \mathrm{H}$ NMR (400 MHz, $\left.\mathrm{CDCl}_{3}\right)$ and ${ }^{13} \mathrm{C}$ NMR (100 MHz, CDCl$)$ spectra of product 3hf
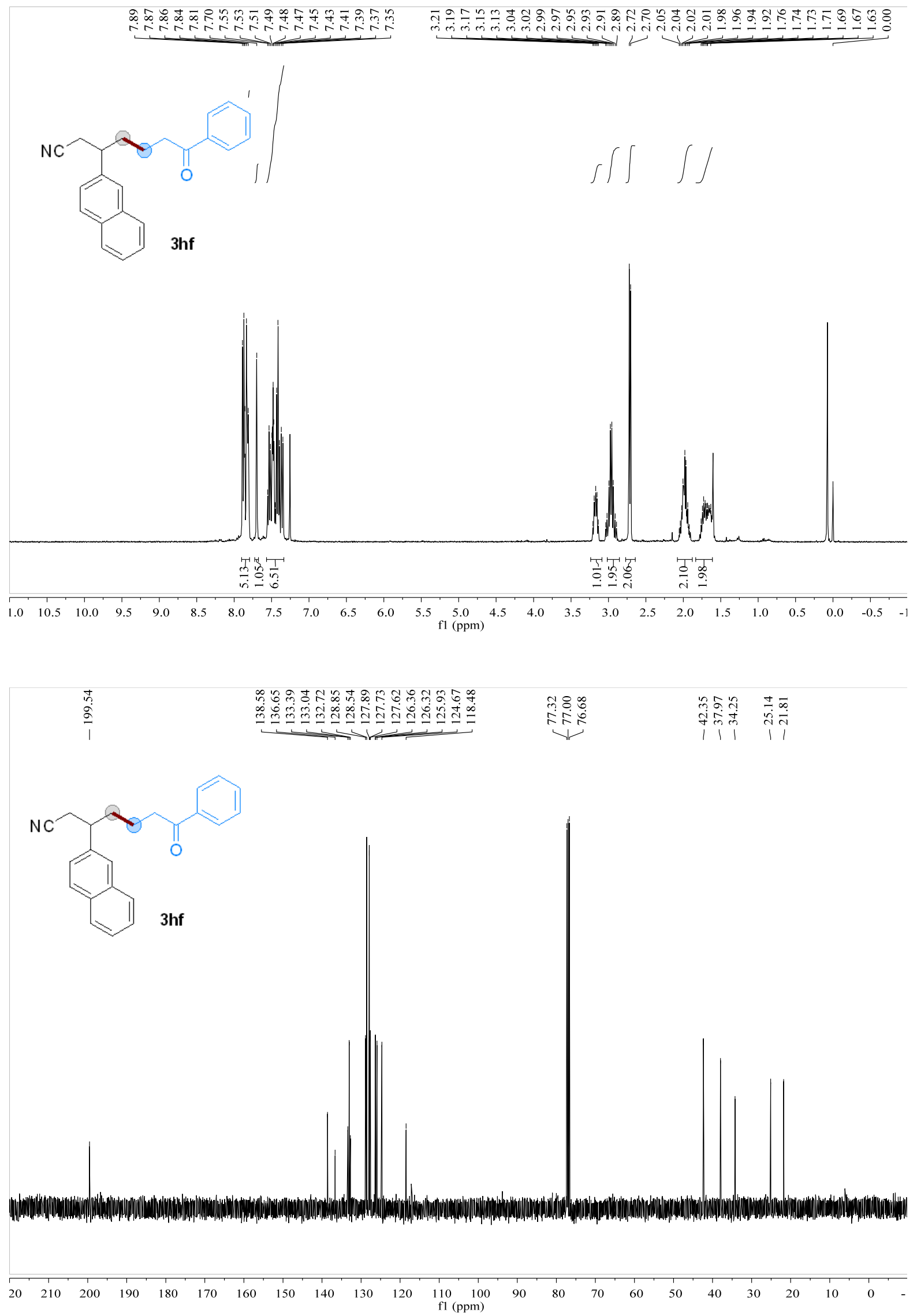


\section{${ }^{1} \mathrm{H}$ NMR (400 $\left.\mathrm{MHz}, \mathrm{CDCl}_{3}\right)$ and ${ }^{13} \mathrm{C}$ NMR (100 MHz, CDCl 3$)$ spectra of product 3hg}

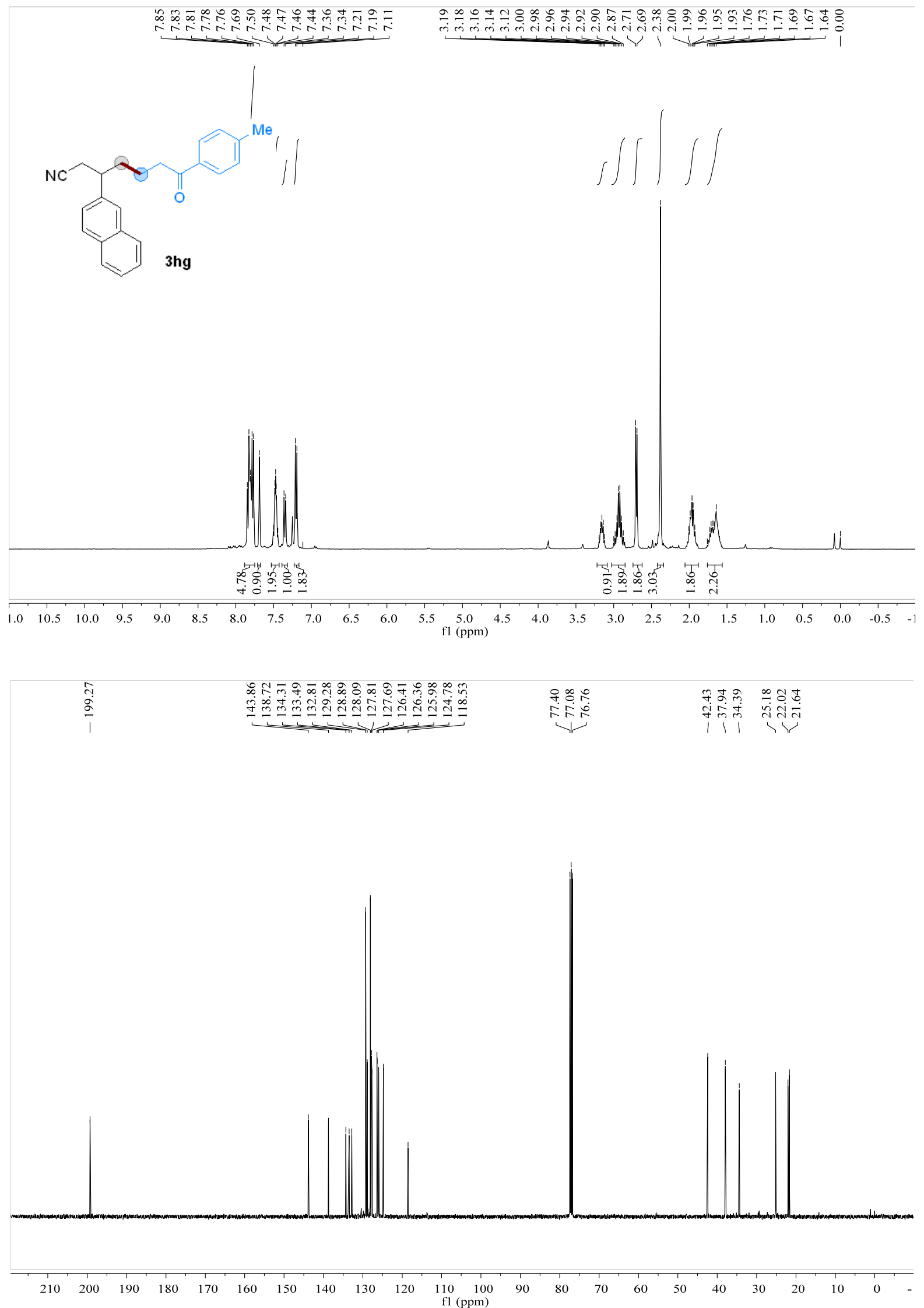




\section{${ }^{1} \mathrm{H}$ NMR (400 MHz, CDCl$)$ and ${ }^{13} \mathrm{C}$ NMR (100 MHz, $\left.\mathrm{CDCl}_{3}\right)$ spectra of product $3 \mathrm{hh}$}
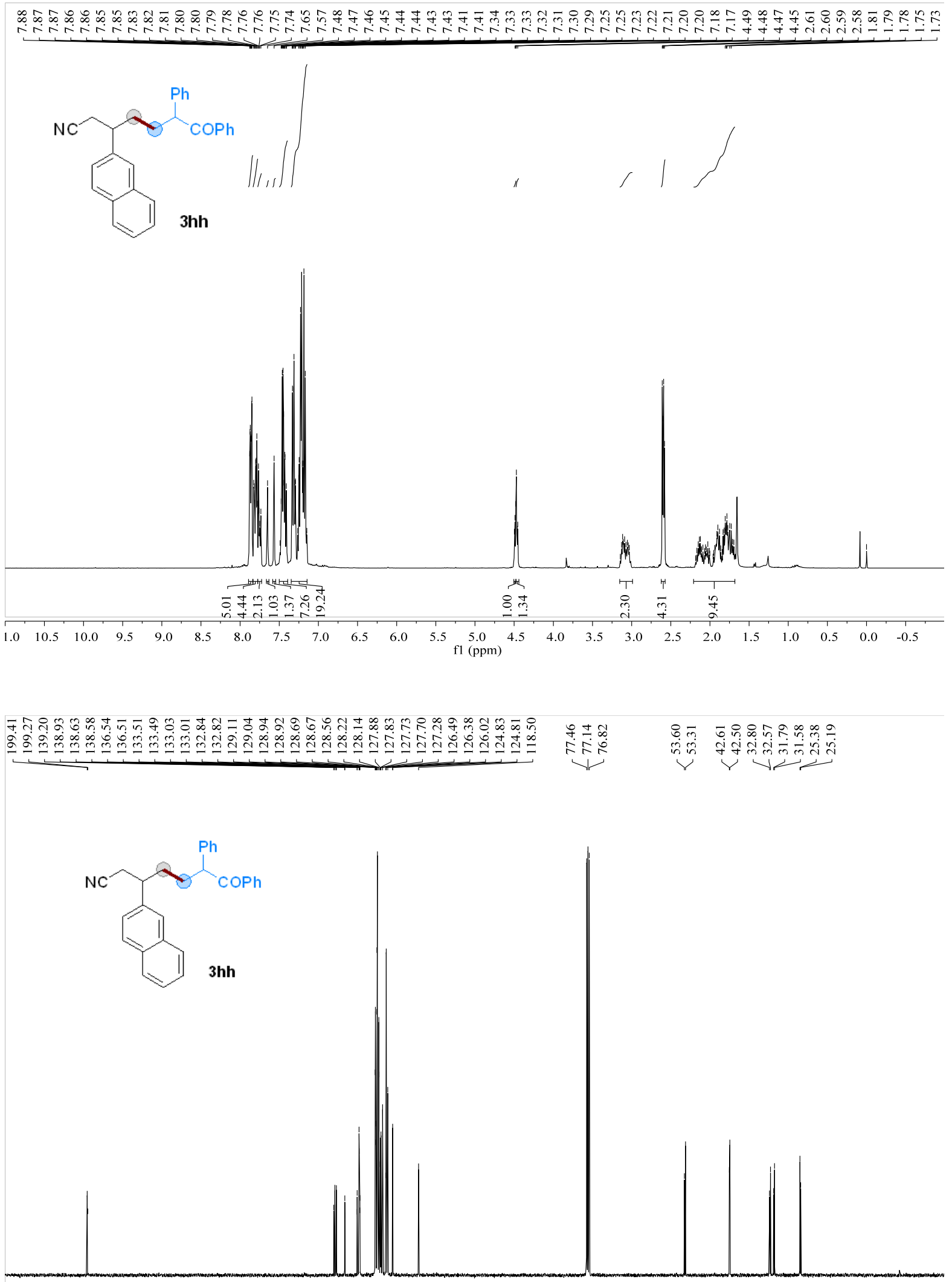

$\begin{array}{llllllllllll}210 & 200 & 190 & 180 & 170 & 160 & 150 & 140 & 130 & 120 & 110 \begin{array}{l}100 \\ \text { fl (ppm) }\end{array}\end{array}$ 
${ }^{1} \mathrm{H}$ NMR (400 MHz, $\left.\mathrm{CDCl}_{3}\right)$ and ${ }^{13} \mathrm{C}$ NMR (100 MHz, CDCl3) spectra of product 3hi

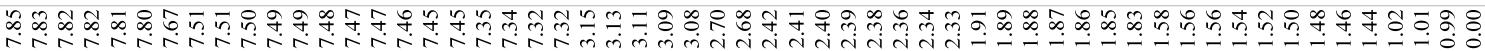
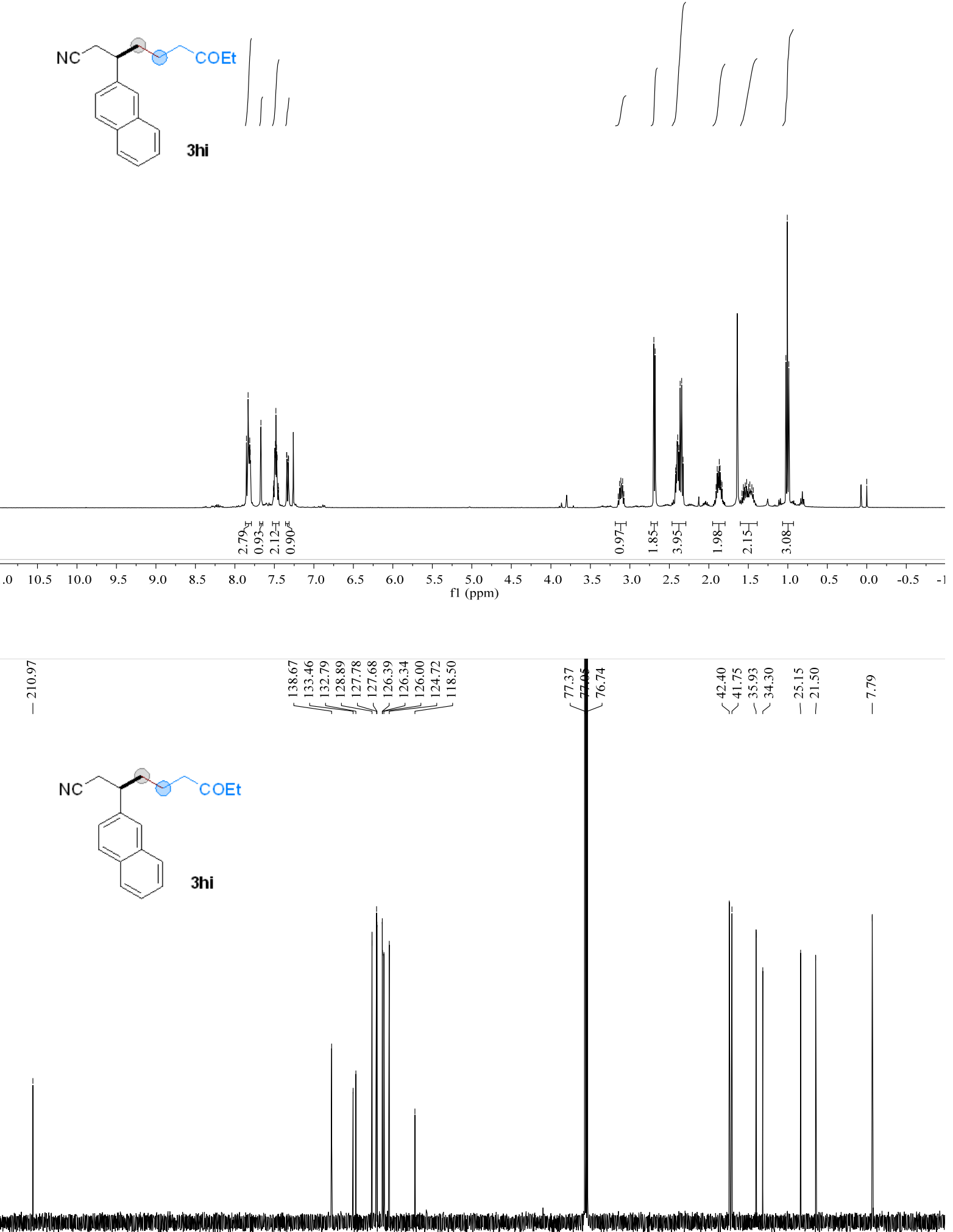

$\begin{array}{llllllllllllllllllllllllll}210 & 200 & 190 & 180 & 170 & 160 & 150 & 140 & 130 & 120 & 110 & 100 & 90 & 80 & 70 & 60 & 50 & 40 & 30 & 20 & 10 & 0 & -\end{array}$ 
${ }^{1} \mathrm{H}$ NMR (400 MHz, $\left.\mathrm{CDCl}_{3}\right)$ and ${ }^{13} \mathrm{C}$ NMR (100 MHz, CDCl3) spectra of product 3hj
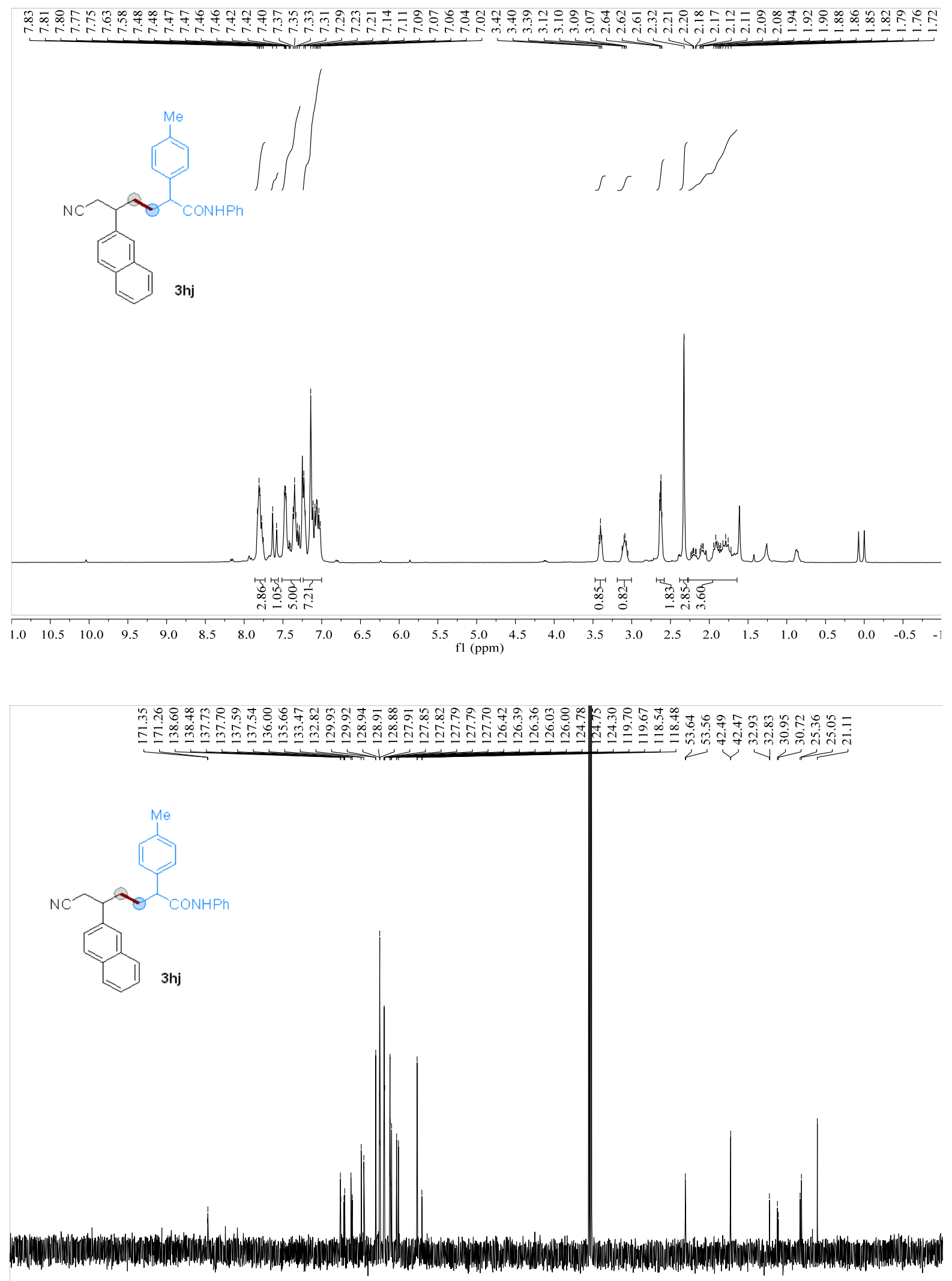

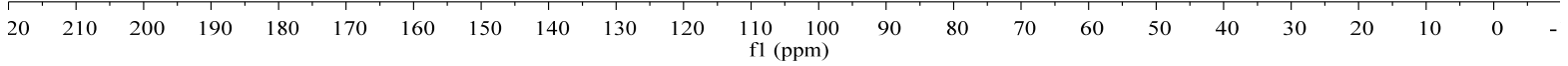

${ }^{1} \mathrm{H}$ NMR (400 MHz, $\left.\mathrm{CDCl}_{3}\right)$ and ${ }^{13} \mathrm{C}$ NMR (100 MHz, $\left.\mathrm{CDCl}_{3}\right)$ spectra of product 3hk 

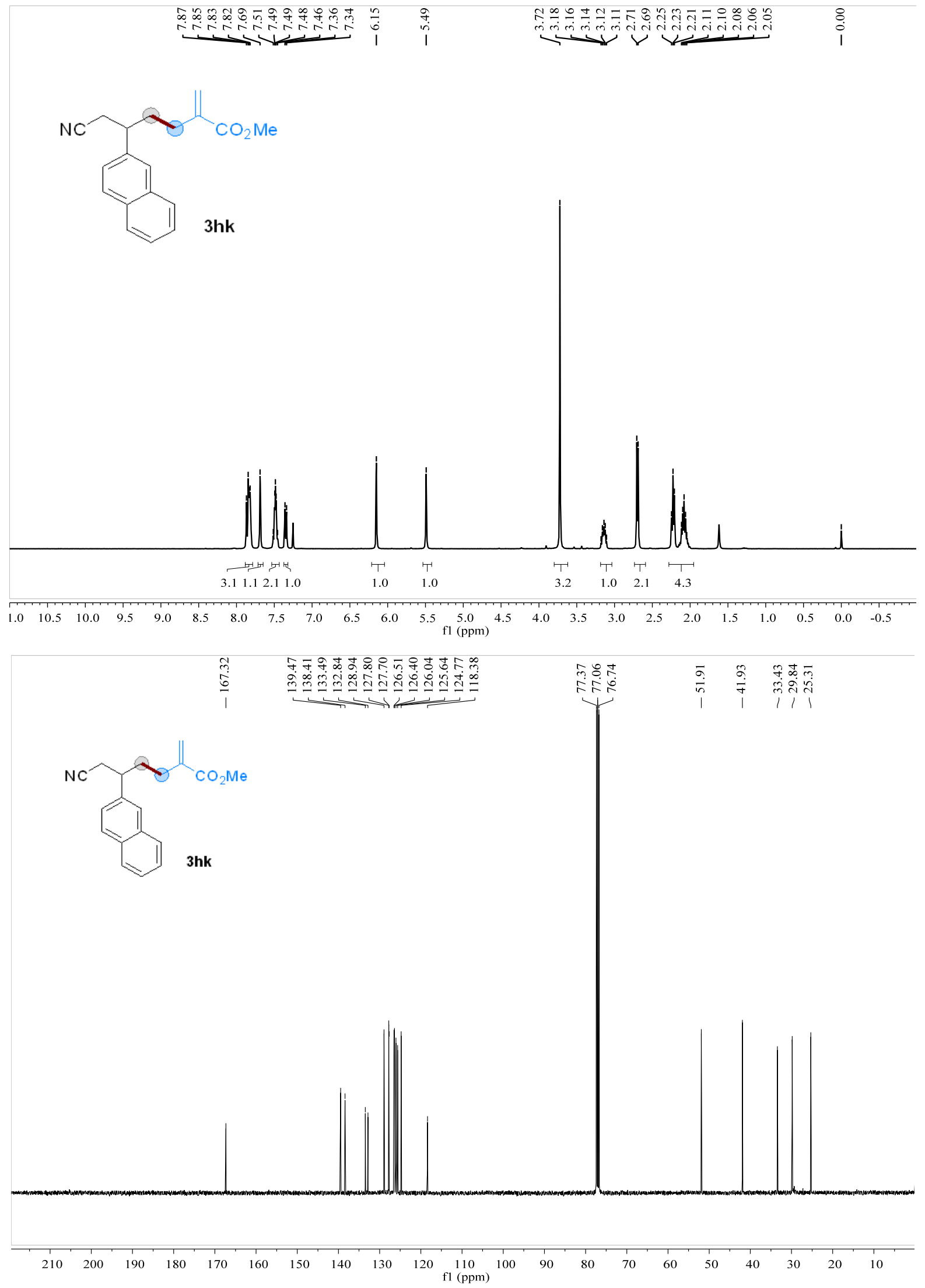

${ }^{1} \mathrm{H}$ NMR (400 MHz, CDCl$)$ and ${ }^{13} \mathrm{C}$ NMR $\left(100 \mathrm{MHz}, \mathrm{CDCl}_{3}\right)$ spectra of product 5 


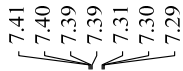

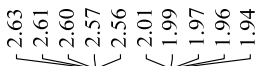<smiles>N#CCCCOC#Cc1ccccc1</smiles>
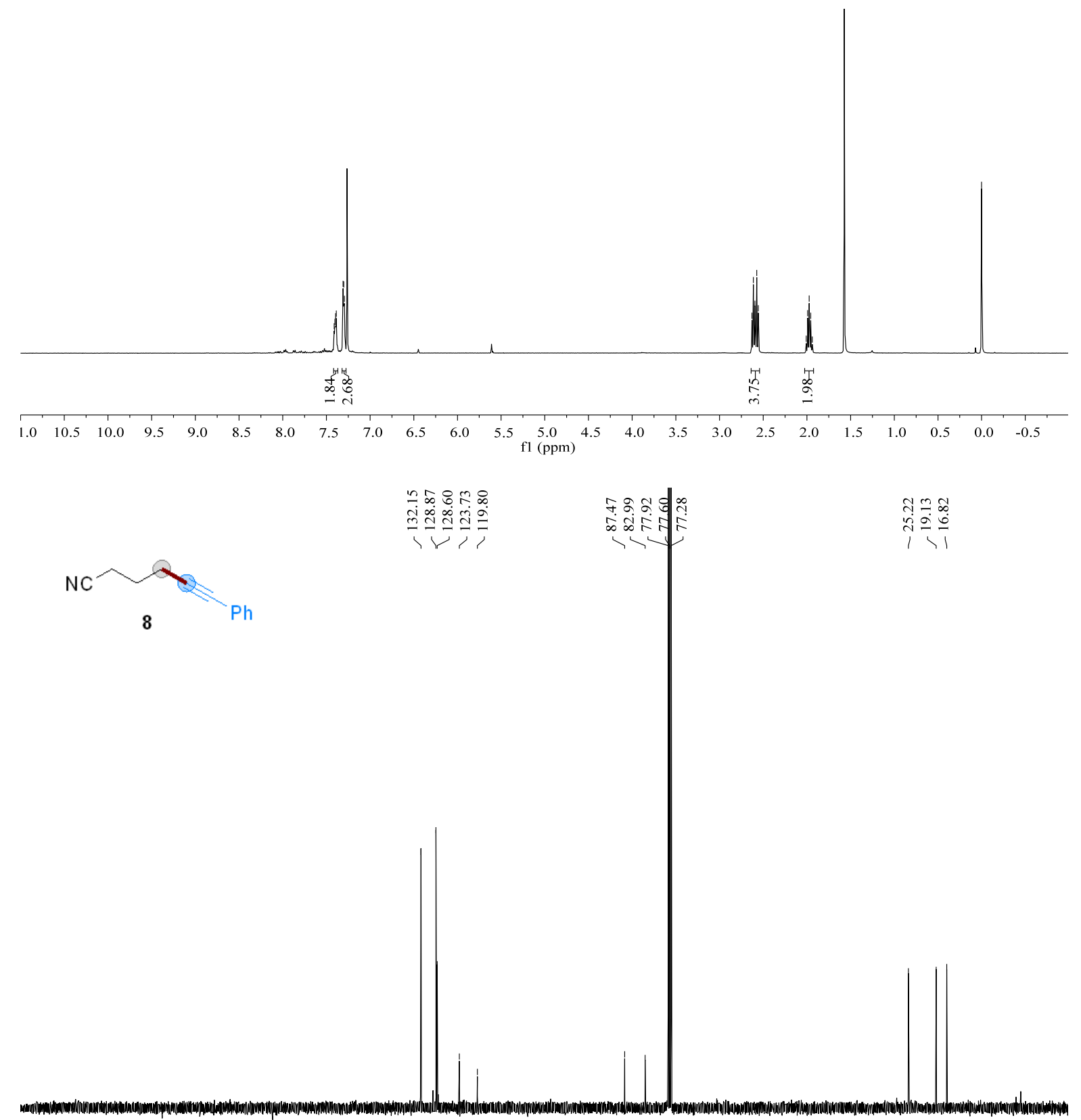

$\begin{array}{llllllllllll}210 & 200 & 190 & 180 & 170 & 160 & 150 & 140 & 130 & 120 & \begin{array}{c}110 \\ \mathrm{f} 1(\mathrm{ppm})\end{array}\end{array}$ 


\section{HPLC Data of Products}

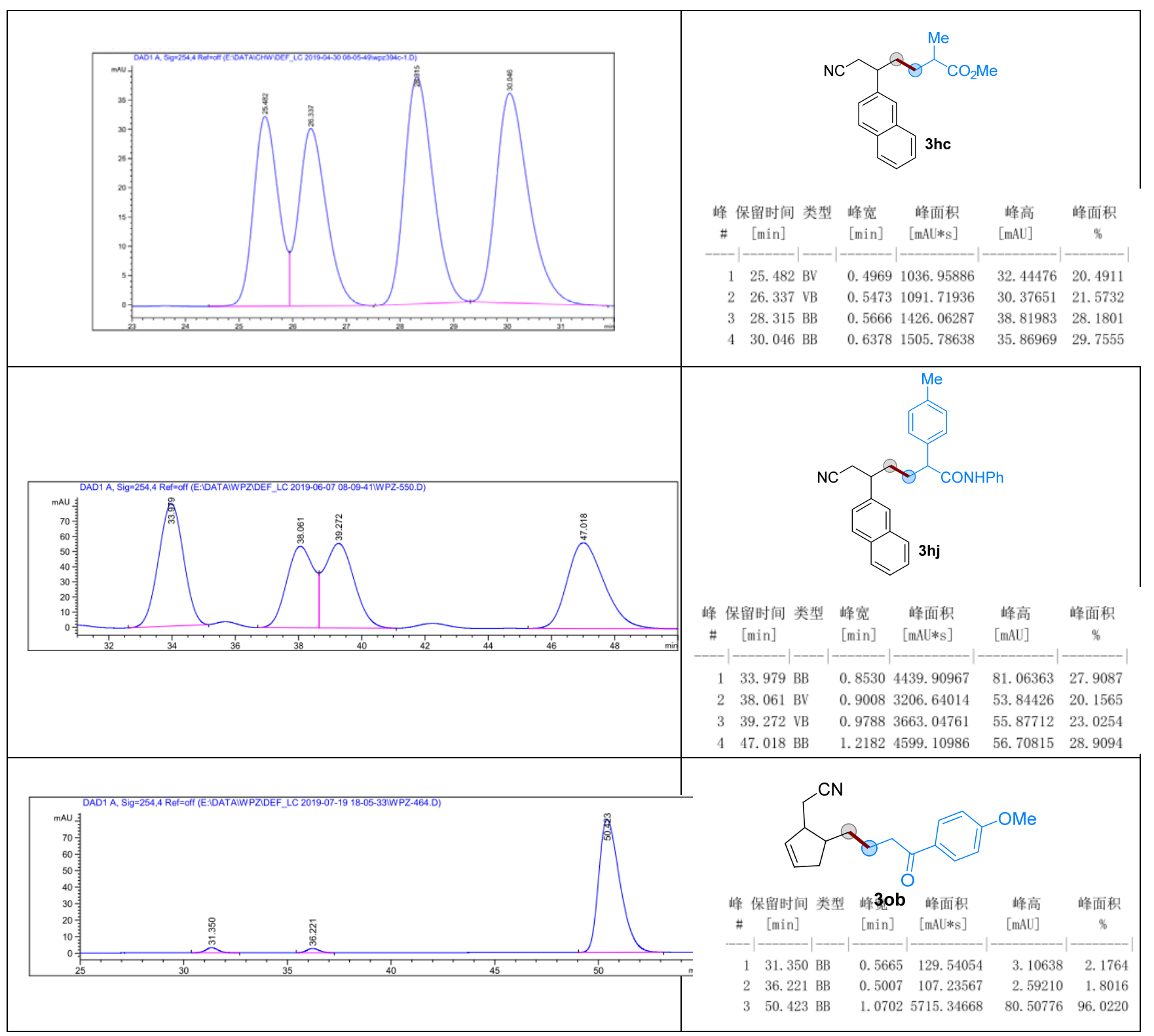

